J. DIFFERENTIAL GEOMETRY

75 (2007) 57-108

\title{
BÄCKLUND TRANSFORMATIONS, WARD SOLITONS, AND UNITONS
}

\author{
Bo Dai \& Chuu-Lian Terng
}

\begin{abstract}
The Ward equation, also called the modified $2+1$ chiral model, is obtained by a dimension reduction and a gauge fixing from the self-dual Yang-Mills field equation on $\mathbb{R}^{2,2}$. It has a Lax pair and is an integrable system. Ward constructed solitons whose extended solutions have distinct simple poles. He also used a limiting method to construct 2-solitons whose extended solutions have a double pole. Ioannidou and Zakrzewski, and Anand constructed more soliton solutions whose extended solutions have a double or triple pole. Some of the main results of this paper are: (i) We construct algebraic Bäcklund transformations (BTs) that generate new solutions of the Ward equation from a given one by an algebraic method. (ii) We use an order $k$ limiting method and algebraic BTs to construct explicit Ward solitons, whose extended solutions have arbitrary poles and multiplicities. (iii) We prove that our construction gives all solitons of the Ward equation explicitly and the entries of Ward solitons must be rational functions in $x, y$ and $t$. (iv) Since stationary Ward solitons are unitons, our method also gives an explicit construction of all $k$-unitons from finite sequences of rational maps from $\mathbb{C}$ to $\mathbb{C}^{n}$.
\end{abstract}

\section{Introduction}

The $2+1$ chiral model is the Euler-Lagrange equation of the functional

$$
\mathcal{E}(J)=\int_{\mathbb{R}^{3}}\left\|J^{-1} J_{x}\right\|^{2}+\left\|J^{-1} J_{y}\right\|^{2}-\left\|J^{-1} J_{t}\right\|^{2} d x d y d t,
$$

where $\|\xi\|^{2}=-\operatorname{tr}\left(\xi^{2}\right), x, y, t$ are the standard space-time variables, and $J$ is a map from the Lorentz space $\mathbb{R}^{2,1}$ to the Lie group $U(n)$. In other words, $J$ is a solution of

$$
\left(J^{-1} J_{t}\right)_{t}-\left(J^{-1} J_{x}\right)_{x}-\left(J^{-1} J_{y}\right)_{y}=0 .
$$

The research of the first author was supported in part by the AMS Fan Fund and a CNSF Grant under no. 10321001. The research of the second author was supported in part by NSF Grant DMS-0306446.

Received 12/02/2004. 
The Ward equation (or the modified $2+1$ chiral model) is the following equation for $J: \mathbb{R}^{2,1} \rightarrow U(n)$ :

$$
\left(J^{-1} J_{t}\right)_{t}-\left(J^{-1} J_{x}\right)_{x}-\left(J^{-1} J_{y}\right)_{y}-\left[J^{-1} J_{t}, J^{-1} J_{y}\right]=0 .
$$

This equation is obtained from a dimension reduction and a gauge fixing of the self-dual Yang-Mills equation on $\mathbb{R}^{2,2}$ (cf. [12]). We call a solution of the Ward equation a Ward map.

A Ward map that is independent of $t$ is a harmonic map from $\mathbb{R}^{2}$ to $U(n)$. If the harmonic map has finite energy, then it extends to a harmonic map from $S^{2}$ to $U(n)$. Such harmonic maps were called unitons, and were studied by Uhlenbeck in [10], Wood in [15], BurstallGuest in [4] and others.

The Ward equation has a Lax pair, i.e., it can be written as the compatibility condition for a system of linear equations involving a spectral parameter $\lambda \in \mathbb{C}$. We explain this next. Let

$$
u=\frac{1}{2}(t+y), \quad v=\frac{1}{2}(t-y) .
$$

Given smooth maps $A, B: \mathbb{R}^{2,1} \rightarrow u(n)$, consider the following linear system for $\psi: \mathbb{R}^{2,1} \times \mathbb{C} \rightarrow G L(n, \mathbb{C})$ :

$$
\left\{\begin{aligned}
\left(\lambda \partial_{x}-\partial_{u}\right) \psi & =A \psi \\
\left(\lambda \partial_{v}-\partial_{x}\right) \psi & =B \psi
\end{aligned}\right.
$$

System (1.4) is overdetermined. Its compatibility condition is

$$
\left[\lambda \partial_{x}-\partial_{u}-A, \lambda \partial_{v}-\partial_{x}-B\right]=0 .
$$

Equate the coefficient of $\lambda^{j}$ in the above equation to get

$$
\left\{\begin{array}{l}
B_{x}=A_{v}, \\
A_{x}-B_{u}-[A, B]=0 .
\end{array}\right.
$$

Suppose $\psi: \mathbb{R}^{2,1} \times \Omega \rightarrow G L(n, \mathbb{C})$ is a smooth solution of (1.4) and satisfies the $U(n)$-reality condition in $\lambda$ :

$$
\psi(x, u, v, \bar{\lambda})^{*} \psi(x, u, v, \lambda)=\mathrm{I},
$$

(i.e., $\psi^{*}=\bar{\psi}^{T}$ ), where $\Omega$ is an open subset of 0 in $\mathbb{C}$. Let

$$
J(x, u, v)=\psi(x, u, v, 0)^{-1} .
$$

Then

$$
A=J^{-1} J_{u}, \quad B=J^{-1} J_{x} .
$$

Thus the compatibility condition (1.5) implies that

$$
\partial_{v}\left(J^{-1} J_{u}\right)=\partial_{x}\left(J^{-1} J_{x}\right) .
$$

Change back to the standard variables $(x, y, t)$ to see that $J$ is a solution to the Ward equation.

A solution $\psi$ to the linear system (1.4) that satisfies the $U(n)$-reality condition is called an extended solution of the Ward equation or extended Ward map, and $J=\psi(\cdots, 0)^{-1}$ is the corresponding Ward map. The 
reality condition for $\psi$ implies that $J$ is unitary. In other words, if we find a $\psi(x, y, t, \lambda)$ so that $\psi$ satisfies the $U(n)$-reality condition and $\left(\lambda \psi_{x}-\psi_{u}\right) \psi^{-1}$ and $\left(\lambda \psi_{v}-\psi_{x}\right) \psi^{-1}$ are independent of $\lambda$, then $J(x, y, t)=$ $\psi(x, y, t, 0)^{-1}$ is a Ward map.

The Ward equation has an infinite number of conservation laws [7]. In particular, the energy functional

$$
E(J)=\frac{1}{2} \iint_{\{t=\text { const }\}}\left\|J^{-1} J_{t}\right\|^{2}+\left\|J^{-1} J_{x}\right\|^{2}+\left\|J^{-1} J_{y}\right\|^{2} d x d y,
$$

is a conserved quantity. To ensure finite energy, Ward imposed the following boundary condition

$$
J=J_{0}+J_{1}(\theta) r^{-1}+O\left(r^{-2}\right) \quad \text { as } r \rightarrow \infty,
$$

where $x+i y=r e^{i \theta}, J_{0}$ is a constant matrix, and $J_{1}$ is independent of $t$.

A Ward map $J$ is called a Ward soliton if $J$

1) has finite energy on $\mathbb{R}^{2}$, or equivalently satisfies the boundary condition (1.8),

2) has an extended solution $\psi$ such that $\psi(x, y, t, \lambda)$ is rational in $\lambda$ and

$$
\lim _{|\lambda| \rightarrow \infty} \psi(x, y, t, \lambda)=\mathrm{I}
$$

for all $(x, y, t)$.

If $\psi$ is an extended solution of the Ward equation with poles at $\lambda=z_{1}, \ldots, z_{r}$ of multiplicities $n_{1}, \ldots, n_{r}$ respectively, then we call $\left(z_{1}, \ldots, z_{r}, n_{1}, \ldots, n_{r}\right)$ the pole data of $\psi$.

Let $z \in \mathbb{C} \backslash \mathbb{R}, \pi$ a Hermitian projection of $\mathbb{C}^{n}$, and

$$
g_{z, \pi}(\lambda)=\pi+\frac{\lambda-\bar{z}}{\lambda-z} \pi^{\perp}=\mathrm{I}+\frac{z-\bar{z}}{\lambda-z} \pi^{\perp} .
$$

A direct computation shows that $g_{z, \pi}$ satisfies the $U(n)$-reality condition (1.6). Such $g_{z, \pi}$ is called a simple element. Uhlenbeck proved in [10] that the set of all simple elements generates the group of rational maps $f: S^{2} \rightarrow G L(n, \mathbb{C})$ that satisfy the $U(n)$-reality condition and $f(\infty)=\mathrm{I}$.

A Ward soliton $J$ is called a $k$-soliton if $k$ is the minimum number of non-constant simple factors of extended solutions of $J$.

Let $z \in \mathbb{C} \backslash \mathbb{R}$ be a fixed constant, $\mathcal{M}_{n \times k}^{0}$ the space of rank $k$ complex $n \times k$ matrices, and $V=\left(v_{i j}\right): \mathbb{C} \rightarrow \mathcal{M}_{n \times k}^{0}$ a meromorphic map. Let $\pi$ denote the map from $\mathbb{R}^{2,1}$ to the space of rank $k$ Hermitian projections of $\mathbb{C}^{n}$ such that $\operatorname{Im}(\pi(x, y, t))$ is the complex linear subspace of $\mathbb{C}^{n}$ spanned by columns of

$$
V\left(x+z u+z^{-1} v\right),
$$

and $\pi^{\perp}=\mathrm{I}-\pi$. Ward (cf. [12]) noted that

$$
g_{z, \pi(x, y, t)}=\mathrm{I}+\frac{z-\bar{z}}{\lambda-z} \pi^{\perp}(x, y, t)
$$


is an extended solution, i.e., a solution of (1.4) with

$$
A=(\bar{z}-z) \pi_{x}, \quad B=(\bar{z}-z) \pi_{v} .
$$

The associated Ward map is

$$
J_{z, V}(x, y, t)=g_{z, V}(x, y, t, 0)^{-1}=\pi(x, y, t)+\frac{z}{\bar{z}} \pi^{\perp}(x, y, t) .
$$

Ward proved that $J_{z, V}$ satisfies the boundary condition (1.8) if and only if each $v_{i j}$ is a rational function (cf. [12]). Hence $J_{z, V}$ is a Ward 1-soliton if each entry of $V$ is a rational function. Note $J_{i, V}$ is a stationary Ward map, i.e., a harmonic map from $\mathbb{C}$ to $U(n)$.

There are several methods for constructing exact Ward multi-solitons: Ward used the method of the Riemann-Hilbert problem with zeros in [12] to construct $k$-soliton solutions whose extended solutions have $k$ simple poles. Such solutions have trivial scattering in the sense that the $k$ one-solitons preserve their travelling directions and shapes after the interaction. Taking the limit of an extended 2-soliton with poles at $i+\epsilon$ and $i-\epsilon$ as $\epsilon \rightarrow 0$, Ward and Ioannidou found extended 2-solitons with a double pole at $\lambda=i$ (cf. $[\mathbf{1 4}, \mathbf{6}, \mathbf{8}])$. Ioannidou also constructed some extended 3 -solitons with a triple pole at $\lambda=i$. These limiting solutions have non-trivial scattering, i.e., the travelling directions of interacting localized lumps change after the interaction. For example, Ioannidou gives examples of extended 2-solitons with a double pole at $\lambda=i$ and with scattering angle $\pi / k$. Anand constructed more solitons with non-trivial scattering in $[\mathbf{1}, \mathbf{2}]$. Ioannidou and Zakrzewski generalized Uhlenbeck's method of adding unitons for harmonic map equation to Ward equation in [8] by writing down an analytic Bäcklund transformation. Vilarroel, Fokas and Ioannidou studied the inverse scattering of the Ward equation in $[\mathbf{1 1}, \mathbf{5}]$. Zhou gave Darboux transformations in [16].

The standard analytic Bäcklund transformation (BT) goes as follows: Given an extended solution $\psi$ of (1.4), if we want to find a projection map $\tilde{\pi}$ so that $\psi_{1}=g_{z, \tilde{\pi}} \psi$ is again an extended solution, then the condition that $\psi_{1}$ satisfies (1.4) for some $\tilde{A}(x, y, t)$ and $\tilde{B}(x, y, t)$ is equivalent to the condition that $\tilde{\pi}$ is a solution of the following system of first order partial differential equations:

$\left(\mathrm{BT}_{z, \psi}\right) \quad\left\{\begin{array}{l}\tilde{\pi}^{\perp}\left(z \tilde{\pi}_{x}-\tilde{\pi}_{u}-A \tilde{\pi}\right)=0, \\ \tilde{\pi}^{\perp}\left(z \tilde{\pi}_{v}-\tilde{\pi}_{x}-B \tilde{\pi}\right)=0,\end{array}\right.$

where $A=\left(\lambda \psi_{x}-\psi_{u}\right) \psi^{-1}$ and $B=\left(\lambda \psi_{v}-\psi_{x}\right) \psi^{-1}$. A solution of $\mathrm{BT}_{z, \psi}$ gives rise to an explicit extended Ward map with one extra pole at $\lambda=z$. Although this first order PDE is solvable, general solutions have not been fully understood. One result of this paper is an explicit construction of all solutions of $\mathrm{BT}_{z, \psi}$ when $\psi$ is an extended Ward soliton. 
Another result of this paper is to construct an algebraic BT for the Ward equation. This is a transformation that generates a new extended solution $\psi_{1}$ by an algebraic formula in terms of a given extended solution $\psi$ and an extended 1-soliton $g_{z, \pi}$. In fact, if $\psi$ is holomorphic and nondegenerate at $\lambda=z$, then

$$
\begin{aligned}
\psi_{1}(x, y, t, \lambda) & =g_{z, \tilde{\pi}(x, y, t)} \psi(x, y, t, \lambda) \\
& =\left(\mathrm{I}+\frac{z-\bar{z}}{\lambda-z} \tilde{\pi}^{\perp}(x, y, t)\right) \psi(x, y, t, \lambda)
\end{aligned}
$$

is also an extended solution of the Ward equation, where $\tilde{\pi}(x, y, t)$ is the Hermitian projection onto $\psi(x, y, t, z) \operatorname{Im}(\pi(x, y, t))$. In other words, $\tilde{\pi}$ is a solution of $\mathrm{BT}_{z, \psi}$. Note that the algebraic BT only works if the given extended solution $\psi$ is holomorphic and non-degenerate at $\lambda=z$. In this case, the new extended solution $\psi_{1}$ has one more pole at $\lambda=z$ than $\psi$. We apply algebraic BTs repeatedly to an extended 1-soliton to get Ward's multi-solitons, whose extended solutions have distinct poles. We use algebraic BTs $k$ times and a delicate limiting method to construct multi-solitons, whose extended solutions have general pole data $\left(z_{1}, \ldots, z_{r}, n_{1}, \ldots, n_{r}\right)$.

There are also analytic and algebraic BTs for harmonic maps from $\mathbb{R}^{2}$ to $U(n)([\mathbf{1 0}, \mathbf{3}])$. But the algebraic BT of a finite energy harmonic map has infinite energy. Hence we cannot produce new harmonic maps on $S^{2}$ using algebraic BTs. Although Uhlenbeck's adding uniton method can be viewed as the limiting case of algebraic BTs as the pole goes to $i$, the limit of these BTs of a harmonic map $s$ gives the same $s$ (for more detail, cf. $[\mathbf{1 0}, \mathbf{3}])$. However, if we apply algebraic BTs of the Ward equation with pole at $i+\epsilon$ to a 1-uniton and choose the projection $\pi_{\epsilon}$ of the Ward 1-soliton $g_{i+\epsilon, \pi_{\epsilon}}$ carefully, then as $\epsilon \rightarrow 0$ the limiting solution can be a 2-uniton. We show in this paper that this limiting method for the Ward equation can produce all unitons into $U(n)$. In fact, we give an explicit construction of $k$-unitons from $k$ rational maps from $\mathbb{C}$ to $\mathbb{C}^{n}$. Our construction of unitons is different from the ones given by Wood in [15] and by Burstall-Guest in [4].

This paper is organized as follows: We give a quick review of unitons and Ward 1-solitons in Section 2, give algebraic Bäcklund transformations for the Ward equation in Section 3. Uhlenbeck proved that a rational map $f: S^{2} \rightarrow G L(n, \mathbb{C})$ satisfying the $U(n)$-reality condition (1.6) and $f(\infty)=\mathrm{I}$ can be factored as a product of simple elements. But such factorization in general is not unique. We give a refinement of this factorization so that it is unique in Section 4. We apply Bäcklund transformations and a careful limiting method to construct Ward solitons that satisfy the boundary condition (1.8) and their extended solutions have pole data $(z, k)$ in Section 5 . We construct multi-solitons 
whose extended solutions have pole data $\left(z_{1}, \ldots, z_{r}, n_{1}, \ldots, n_{r}\right)$ in Section 6. We show in Section 7 that the first equation of $\mathrm{BT}_{z, \psi}$ defines a natural complex structure on the trivial bundle $S^{2} \times \mathbb{C}^{n}$ over $S^{2}$, and a solution of $\mathrm{BT}_{z, \psi}$ corresponds to a holomorphic subbundle of the trivial bundle that satisfies certain first order PDE constraint. In section 8 , we use the holomorphic vector bundle formulation of Section 7 to prove that algebraic BTs and the limiting method of Section 5 produce all solutions of $\mathrm{BT}_{z, \psi}$ for any extended Ward-soliton $\psi$; hence we can construct all Ward solitons explicitly. In Section 9, we give an explicit construction of all unitons using the limiting method of Section 5. Finally, in Section 10, we graphically give the wave profiles of some Ward $k$-solitons by showing the graph of their energy density $E\left(x, y, t_{i}\right)$ for a sequence of increasing $t_{i}$. Results in Section 6 tell us that a soliton with general pole data $\left(z_{1}, \ldots, z_{r}, n_{1}, \ldots, n_{r}\right)$ can be constructed by simple algebraic methods from the $n_{i}$-solitons with pole data $\left(z_{i}, n_{i}\right)$. The graphics in the last section indicate that a Ward soliton with pole data $\left(z_{1}, \ldots, z_{k}, n_{1}, \ldots, n_{k}\right)$ is the interaction of $k$ Ward solitons with pole data $\left(z_{1}, n_{1}\right), \ldots,\left(z_{k}, n_{k}\right)$ respectively and these $k$ solitons keep their shapes after interaction. But the dynamics of solitons with pole data $(z, k)$ are intriguing, quite complicated, and deserve further investigation.

The reader can play the Quick Time movies for the Figures given in the last section by going to

$$
\text { http://math.uci.edu/ cterng/WardSolitonMovies.html }
$$

The authors would like to thank Richard Palais for helping them write the codes for the Ward multi-solitons (Object Pascal), and use his computer program 3D-XplorMath to show the corresponding wave motions. The first author would like to thank the AMS Fan Fund and Northeastern University for sponsoring his visit to Northeastern University during the spring quarter of 2003, where the cooperation started. The second author thanks Karen Uhlenbeck for many useful discussions, and thanks MSRI for supporting her visit during the winter quarter of 2004, where she worked on this paper.

\section{1-unitons and 1-soliton Ward maps}

A stationary solution of the Ward equation is a harmonic map from $\mathbb{R}^{2}$ to $U(n)$. If in addition it has finite energy then it is a harmonic map from $S^{2}$. All such harmonic maps are called unitons, which are studied by Uhlenbeck [10], Wood [15], Burstall-Guest [4] and others.

The harmonic map equation is integrable in the sense that there is an associated linear system with a complex parameter $\xi \in \mathbb{C} \backslash\{0\}$. Namely, if $s$ is a harmonic map from $\mathbb{C}$ to $U(n)$, then the following linear system 
is compatible:

$$
\left\{\begin{array}{l}
E_{z}=\left(1-\xi^{-1}\right) E P \\
E_{\bar{z}}=-(1-\xi) E P^{*}
\end{array}\right.
$$

where $P=\frac{1}{2} s^{-1} s_{z}$. Note that the compatibility condition of system (2.1) is the harmonic map equation,

$$
P_{\bar{z}}=-\left[P, P^{*}\right] \text {. }
$$

Conversely, if $E(x, y, \xi)$ is a solution of $(2.1)$ and satisfies the $U(n)$ reality condition (1.6), then $s(x, y)=E(x, y,-1)$ is a harmonic map. Such $E$ is called an extended solution of the harmonic map equation. A direct computation implies that if $E(x, y, \xi)$ is an extended solution of the harmonic map equation, then

$$
\psi(x, y, t, \lambda)=E\left(x, y, \frac{\lambda-i}{\lambda+i}\right)^{-1}
$$

is an extended solution of the Ward equation, i.e., $\psi$ is a solution of (1.4) and $\psi(x, y, t, 0)^{-1}=s(x, y)$ is a stationary Ward map.

Let $V=\left(v_{i j}\right): \mathbb{C} \rightarrow \mathcal{M}_{n \times k}^{0}(\mathbb{C})$ be a rational map, $\pi$ the projection of $\mathbb{C}^{n}$ onto the subspace spanned by the $k$ columns of $V$, and $\pi^{\perp}=\mathrm{I}-\pi$. Then $s=\pi-\pi^{\perp}$ is a 1-uniton. Moreover, all 1-unitons are of this form. The 1-uniton $\pi-\pi^{\perp}$ has an extended solution:

$$
E(x, y, \xi)=\pi(x, y)+\xi \pi(x, y)^{\perp} .
$$

Uhlenbeck proved in [10] that given a harmonic map $s: S^{2} \rightarrow U(n)$, there exists an extended solution $E(x, y, \xi)$ of the form

$$
E(x, y, \xi)=\left(\pi_{1}+\xi \pi_{1}^{\perp}\right) \cdots\left(\pi_{k}+\xi \pi_{k}^{\perp}\right),
$$

where each $\pi_{i}(x, y)$ is a projection onto some $k_{i}$-dimensional linear subspace $V_{i}(x, y)$ of $\mathbb{C}^{n}$ and $k \leq(n-1)$. Such solutions are called $k$-unitons.

Substitute $\xi=\frac{\lambda-i}{\lambda+i}$ into $(2.3)$ to get an extended Ward $k$-soliton with pole data $(i, k)$. In particular,

$$
\begin{aligned}
\psi(x, y, t, \lambda) & =E\left(x, y, \frac{\lambda+i}{\lambda-i}\right) \\
& =\pi(x, y)+\frac{\lambda+i}{\lambda-i} \pi^{\perp}(x, y)=g_{i, \pi(x, y)}(\lambda),
\end{aligned}
$$

is an extended Ward 1-soliton with a simple pole at $\lambda=i$.

A general extended Ward 1-soliton (1.9) is obtained by replacing $i$ with a non-real complex constant $z$ and $x+i y$ with $w=x+z u+z^{-1} v$. The associated Ward map is

$$
\hat{J}_{z, V}(x, y, t)=\psi(x, y, t, 0)^{-1}=\pi+\frac{z}{\bar{z}} \pi^{\perp} .
$$


Note that $\hat{J}_{z, V}$ has constant determinant $(z / \bar{z})^{n-k}$. So we can normalize it to get a Ward map into $S U(n)$ :

$$
J_{z, V}(x, y, t)=\left(\frac{\bar{z}}{z}\right)^{\frac{n-k}{n}}\left(\pi+\frac{z}{\bar{z}} \pi^{\perp}\right)=\left(\frac{z}{\bar{z}}\right)^{\frac{k}{n}}\left(\frac{\bar{z}}{z} \pi+\pi^{\perp}\right) .
$$

The 1-soliton $J_{z, V}$ is a travelling wave. To see this, write $z=r e^{i \theta}$ and compute directly to get

$$
w=x+z u+z^{-1} v=\left(x-v_{1} t\right)+k_{1}\left(y-v_{2} t\right)+i k_{2}\left(y-v_{2} t\right),
$$

where $v_{1}=-\frac{2 r \cos \theta}{1+r^{2}}, v_{2}=\frac{1-r^{2}}{1+r^{2}}$, and $k_{1}+i k_{2}=\left(z-z^{-1}\right) / 2$. Thus $J_{z, V}$ is a travelling wave with constant velocity

$$
\vec{v}=\left(-\frac{2 r \cos \theta}{1+r^{2}}, \frac{1-r^{2}}{1+r^{2}}\right)
$$

on the $x y$-plane.

Example 2.1 (Ward 1-solitons). Let $z \in \mathbb{C} \backslash \mathbb{R}, f: \mathbb{C} \rightarrow \mathbb{C}$ be a rational function, $w=x+z u+z^{-1} v, V(w)=\left(\begin{array}{c}1 \\ f(w)\end{array}\right)$, and $\pi(x, y, t)$ the projection onto $\mathbb{C} V(w)$. A direct computation gives

$$
J_{z, V}=\frac{1}{|z|\left(1+|f(w)|^{2}\right)}\left(\begin{array}{cc}
\bar{z}+z|f(w)|^{2} & (\bar{z}-z) \overline{f(w)} \\
(\bar{z}-z) f(w) & \bar{z}|f(w)|^{2}+z
\end{array}\right) .
$$

\section{Algebraic Bäcklund transformations}

In this section, we give an algebraic BT to construct a family of explicit solutions from a given extended solution $\psi(\lambda)(x, y, t)=\psi(x, y, t, \lambda)$ of the Ward equation.

Theorem 3.1 (Algebraic Bäcklund transformation). Let $\psi(x, y, t, \lambda)$ be an extended solution of the Ward equation, and $J=\psi(\cdots, 0)^{-1}$ the associated Ward map. Choose $z \in \mathbb{C} \backslash \mathbb{R}$ such that $\psi$ is holomorphic and non-degenerate at $\lambda=z$. Let $g_{z, \pi(x, y, t)}(\lambda)$ be an extended 1-soliton, and $\tilde{\pi}(x, y, t)$ the Hermitian projection of $\mathbb{C}^{n}$ onto

$$
\psi(x, y, t, z)(\operatorname{Im}(\pi(x, y, t)) .
$$

Then

1) $\tilde{\psi}(x, y, t, \lambda)=g_{z, \tilde{\pi}(x, y, t)}(\lambda) \psi(x, y, t, \lambda) g_{z, \pi(x, y, t)}(\lambda)^{-1}$ is holomorphic and non-degenerate at $\lambda=z, \bar{z}$,

2) $\psi_{1}=g_{z, \tilde{\pi}} \psi=\tilde{\psi} g_{z, \pi}$ is a new extended solution to the linear system (1.4) with

$$
(A, B) \rightarrow\left(A+(\bar{z}-z) \tilde{\pi}_{x}, B+(\bar{z}-z) \tilde{\pi}_{v}\right),
$$

and the new Ward map is

$$
J_{1}(x, y, t)=\left(\frac{z}{\bar{z}}\right)^{k / n} J(x, y, t)\left(\frac{\bar{z}}{z} \tilde{\pi}(x, y, t)+\tilde{\pi}^{\perp}(x, y, t)\right) .
$$


Proof.

(1) Let $\tilde{\psi}(\lambda)=g_{z, \tilde{\pi}}(\lambda) \psi(\lambda) g_{z, \pi}(\lambda)^{-1}$. Then residue calculus implies that $\tilde{\psi}(\lambda)$ is holomorphic at $\lambda=z, \bar{z}$. Thus we have two factorizations of $\psi_{1}=g_{z, \tilde{\pi}} \psi=\tilde{\psi} g_{z, \pi}$.

(2) It suffices to show that

$$
A_{1}:=\left(\lambda \partial_{x} \psi_{1}-\partial_{u} \psi_{1}\right) \psi_{1}^{-1}
$$

is independent of $\lambda$. Using $\psi_{1}=g_{z, \tilde{\pi}} \psi$, we have

$$
A_{1}=\left(\lambda \partial_{x} g_{z, \tilde{\pi}}-\partial_{u} g_{z, \tilde{\pi}}\right) g_{z, \tilde{\pi}}^{-1}+g_{z, \tilde{\pi}}\left(\lambda \partial_{x} \psi-\partial_{u} \psi\right) \psi^{-1} g_{z, \tilde{\pi}}^{-1} .
$$

Since $\left(\lambda \partial_{x} \psi-\partial_{u} \psi\right) \psi^{-1}$ is constant in $\lambda$ by assumption, (3.2) is holomorphic at $\lambda \in \mathbb{C} \backslash\{z, \bar{z}\}$, and has at most a simple pole at $\lambda=\infty$. But

$$
\operatorname{Res}_{\lambda=\infty} A_{1}=\left.\left(\partial_{x} g_{z, \tilde{\pi}}\right) g_{z, \tilde{\pi}}^{-1}\right|_{\lambda=\infty}=0
$$

as $g_{z, \tilde{\pi}}(\infty) \equiv \mathrm{I}$. So $A_{1}$ is holomorphic at $\lambda \in \mathbb{C} \cup\{\infty\} \backslash\{z, \bar{z}\}$. On the other hand, using $\psi_{1}=\tilde{\psi} g_{z, \pi}$,

$$
A_{1}=\left(\lambda \partial_{x} \tilde{\psi}-\partial_{u} \tilde{\psi}\right) \tilde{\psi}^{-1}+\tilde{\psi}\left(\lambda \partial_{x} g_{z, \pi}-\partial_{u} g_{z, \pi}\right) g_{z, \pi}^{-1} \tilde{\psi}^{-1}
$$

Since $\left(\lambda \partial_{x} g_{z, \pi}-\partial_{u} g_{z, \pi}\right) g_{z, \pi}^{-1}$ is independent of $\lambda, A_{1}$ is holomorphic at $\lambda=z, \bar{z}$. Thus we see that $A_{1}$ is holomorphic on $\mathbb{C} \cup\{\infty\}$, hence independent of $\lambda$ by Liouville's Theorem. Likewise $\left(\lambda \partial_{v} \psi_{1}-\partial_{x} \psi_{1}\right) \psi_{1}^{-1}$ is also independent of $\lambda$. The remaining computation is straightforward.

q.e.d.

Let

$$
\psi_{1}=g_{z, \pi} * \psi, \quad J_{1}=g_{z, \pi} * J
$$

denote the algebraic Bäcklund transformation generated by $g_{z, \pi}$. If we apply BTs repeatedly (with distinct poles) to an extended 1-soliton solution, then we obtain Ward multi-solitons, whose extended solutions have only simple poles. Such solutions coincide with the ones obtained by Ward [12] using solutions of the Riemann-Hilbert problem.

Example 3.2 (Ward 2-solitons with trivial scattering). Let $z_{1}, z_{2}$ be two distinct complex numbers and $z_{1} \neq \bar{z}_{2}, f_{1}, f_{2}: \mathbb{C} \rightarrow \mathbb{C}$ rational functions, and $\pi_{i}(x, y, t)$ the projection onto $\mathbb{C}\left(\begin{array}{c}1 \\ f_{i}\left(w_{i}\right)\end{array}\right)$, where

$$
w_{i}=x+z_{i} u+z_{i}^{-1} v, \quad i=1,2 .
$$

Then $g_{z_{1}, \pi_{1}}$ and $g_{z_{2}, \pi_{2}}$ are extended 1-soliton solutions of the Ward equation. Apply Bäcklund transformation (Theorem 3.1) with $\psi=g_{z_{1}, \pi_{1}}$ and $g_{z, \pi}=g_{z_{2}, \pi_{2}}$. Compute directly to see that $\psi\left(z_{2}\right)\left(\left(\begin{array}{c}1 \\ f_{2}\left(w_{2}\right)\end{array}\right)\right)$ is 
parallel to

$$
\tilde{v}_{2}=A\left(\begin{array}{c}
1 \\
f_{1}\left(w_{1}\right)
\end{array}\right)+B\left(\begin{array}{c}
\overline{f_{1}\left(w_{1}\right)} \\
-1
\end{array}\right)
$$

where

$$
A=1+\overline{f_{1}\left(w_{1}\right)} f_{2}\left(w_{2}\right), \quad B=\frac{z_{2}-\bar{z}_{1}}{z_{2}-z_{1}}\left(f_{1}\left(w_{1}\right)-f_{2}\left(w_{2}\right)\right) .
$$

The new extended solution of the Ward equation is

$$
\tilde{\psi}=g_{z_{2}, \tilde{\pi}_{2}} g_{z_{1}, \pi_{1}},
$$

where $\tilde{\pi}_{2}$ is the projection onto $\mathbb{C} \tilde{v}_{2}$. The associated Ward map is

$$
J=c\left(\pi_{1}+\frac{z_{1}}{\bar{z}_{1}} \pi_{1}^{\perp}\right)\left(\tilde{\pi}_{2}+\frac{z_{2}}{\bar{z}_{2}} \tilde{\pi}_{2}^{\perp}\right)
$$

where $c^{2}=\frac{\bar{z}_{1} \bar{z}_{2}}{z_{1} z_{2}}$ is a normalizing constant to make $\operatorname{det}(J)=1$.

Remark. If $J$ is a Ward map into $S U(n)$, then the Ward map associated to $\psi_{1}$ in Theorem 3.1 is

$$
\hat{J}_{1}=J\left(\tilde{\pi}+\frac{z}{\bar{z}} \tilde{\pi}^{\perp}\right),
$$

which is a Ward map into $U(n)$. But $\operatorname{det}\left(\hat{J}_{1}\right)=(z / \bar{z})^{n-k}$ is constant. So $J_{1}=(\bar{z} / z)^{\frac{n-k}{k}} \hat{J}_{1}$ is a Ward map into $S U(n)$. This means that BTs are defined for both the $S U(n)$ and the $U(n)$ case.

\section{Minimal factorization}

Let $\mathcal{S}_{r}\left(S^{2}, G L(n)\right)$ denote the group of rational maps $f: S^{2} \rightarrow$ $G L(n, \mathbb{C})$ that satisfies the reality condition $f(\bar{\lambda})^{*} f(\lambda)=\mathrm{I}$ and $f(\infty)=$ I. First we recall the factorization theorem of Uhlenbeck [10]

Theorem $4.1([\mathbf{1 0}])$. The group $\mathcal{S}_{r}\left(S^{2}, G L(n)\right)$ is generated by the set of all simple elements, i.e., every $f \in \mathcal{S}_{r}\left(S^{2}, G L(n)\right)$ can be factored as a product of simple elements,

$$
f=g_{z_{1}, \pi_{1}} \cdots g_{z_{k}, \pi_{k}},
$$

for some $z_{1}, \ldots, z_{k}$ and Hermitian projections $\pi_{1}, \ldots, \pi_{k}$.

However, the above factorization is not unique. For example, if $\operatorname{Im} \pi_{1}$ is orthogonal to $\operatorname{Im} \pi_{2}$ then $g_{z, \pi_{1}^{\perp}} g_{z, \pi_{2}^{\perp}}=g_{z, \pi^{\perp}}$, where $\pi$ is the projection onto $\operatorname{Im} \pi_{1} \oplus \operatorname{Im} \pi_{2}$. Moreover, if $z_{1} \neq z_{2}, \bar{z}_{2}$, then $g_{z_{1}, \pi_{1}} g_{z_{2} \pi_{2}}$ can be written as $g_{z_{2}, \tau_{2}} g_{z_{1}, \tau_{1}}$ for some projections $\tau_{1}, \tau_{2}$. This is the permutability formula for simple elements given in Theorem 6.2 of [9], which can be reformulated as follows: 
Theorem $4.2([9])$. Suppose $z_{1} \neq z_{2}, \bar{z}_{2}$, and $\pi_{1}, \pi_{2}$ are Hermitian projections of $\mathbb{C}^{n}$. Let $\tilde{\pi}_{1}$ be the projection onto $g_{z_{2}, \pi_{2}}\left(z_{1}\right)\left(\operatorname{Im} \pi_{1}\right)$, and $\tilde{\pi}_{2}$ the projection onto $g_{z_{1}, \pi_{1}}\left(z_{2}\right)\left(\operatorname{Im} \pi_{2}\right)$. Then

$$
g_{z_{1}, \tilde{\pi}_{1}} g_{z_{2}, \pi_{2}}=g_{z_{2}, \tilde{\pi}_{2}} g_{z_{1}, \pi_{1}} .
$$

Conversely, if $\tau_{i}$ are projections so that $g_{z_{1}, \tau_{1}} g_{z_{2}, \pi_{2}}=g_{z_{2}, \tau_{2}} g_{z_{1}, \pi_{1}}$, then $\tau_{i}=\tilde{\pi}_{i}$ for $i=1,2$.

Recall that $g_{z, \pi} * \psi$ is the algebraic BT of $\psi$ generated by the 1-soliton $g_{z, \pi}$. As a consequence of Theorem 4.2 we have

Corollary 4.3. If $g_{z_{i}, \pi_{i}}$ are extended Ward 1-solitons, then

$$
g_{z_{2}, \pi_{2}} * g_{z_{1}, \pi_{1}}=g_{z_{1}, \pi_{1}} * g_{z_{2}, \pi_{2}} .
$$

Note that the proof of Theorem 3.1 (1) gives a more general permutability formula:

Proposition 4.4. Suppose $f \in \mathcal{S}_{r}\left(S^{2}, G L(n)\right)$ is holomorphic and non-degenerate at $\lambda=z$ and $g_{z, \pi}$ is a simple element. Let $\tilde{\pi}$ be the projection onto $f(z)(\operatorname{Im} \pi)$. Then

1) $\tilde{f}=g_{z, \tilde{\pi}} f g_{z, \pi}^{-1}$ is holomorphic at $\lambda=z, \bar{z}$,

2) $\tilde{f} g_{z, \pi}=g_{z, \tilde{\pi} f}$.

As a consequence of the factorization Theorem 4.1 and permutability formula 4.2 we have

Corollary 4.5. Let $f \in \mathcal{S}_{r}\left(S^{2}, G L(n)\right)$, and $C(f)$ be the set of poles of $f$. Suppose $C(f) \subset \mathbb{C}_{+}=\{r+i s \mid s>0\}, C_{1}, C_{2}$ are proper disjoint subsets of $C(f)$, and $C(f)=C_{1} \cup C_{2}$. Then there exist unique $f_{1}, f_{2} \in$ $\mathcal{S}_{r}\left(S^{2}, G L(n)\right)$ so that $f=f_{1} f_{2}$ and $C\left(f_{i}\right)=C_{i}$ for $i=1,2$.

Corollary 4.6. Let $z_{i} \in \mathbb{C}_{+}$for $1 \leq i \leq k$, and $f \in \mathcal{S}_{r}\left(S^{2}, G L(n)\right)$ with pole data $\left(z_{1}, \ldots, z_{k}, n_{1}, \ldots, n_{k}\right)$. Then:

1) There exist unique $f_{i} \in \mathcal{S}_{r}\left(S^{2}, G L(n)\right)$ with pole data $\left(z_{i}, n_{i}\right)$ and $F_{i} \in \mathcal{S}_{r}\left(S^{2}, G L(n)\right)$ that is holomorphic and non-degenerate at $z_{i}, \bar{z}_{i}$ such that $f=F_{i} f_{i}$ for each $i$.

2) There exist $g_{j} \in \mathcal{S}_{r}\left(S^{2}, G L(n)\right)$ with pole data $\left(z_{j}, n_{j}\right)$ for $1 \leq j \leq$ $k$ so that $f=g_{1} \cdots g_{k}$.

Next we give a refinement of the factorization for $\phi \in \mathcal{S}_{r}\left(S^{2}, G L(n)\right)$ with pole data $(z, k)$. First we need a lemma.

Lemma 4.7. Let $\pi_{1}$ and $\pi_{2}$ be two Hermitian projections of $\mathbb{C}^{n}$ onto $V_{1}, V_{2}$ respectively.

1) If $V_{1} \perp V_{2}$, then

$$
g_{z, \pi_{2}} g_{z, \pi_{1}}=\frac{\lambda-\bar{z}}{\lambda-z} g_{z, \tau}
$$

where $\tau=\pi_{2}+\pi_{1}$ is the projection onto $V_{2} \oplus V_{1}$. 
2) Suppose $V_{2}^{1}:=V_{2} \cap V_{1}^{\perp} \neq 0$. Let $\tau_{2}$ and $\tau_{1}$ be the projections onto $V_{2} \cap\left(V_{2}^{1}\right)^{\perp}$ and $V_{1} \oplus V_{2}^{1}$ respectively. Then $\operatorname{Im} \tau_{2} \cap \operatorname{Im} \tau_{1}^{\perp}=0$ and

$$
g_{z, \pi_{2}} g_{z, \pi_{1}}=g_{z, \tau_{2}} g_{z, \tau_{1}} \text {. }
$$

Proof. A direct computation gives (4.1) and (4.2). Compute directly to see

$$
\begin{aligned}
\left(\operatorname{Im} \tau_{2}\right) \cap\left(\operatorname{Im} \tau_{1}^{\perp}\right) & =\left(V_{2} \cap\left(V_{2}^{1}\right)^{\perp}\right) \cap\left(V_{1} \oplus V_{2}^{1}\right)^{\perp} \\
& =\left(V_{2} \cap\left(V_{2}^{1}\right)^{\perp}\right) \cap\left(V_{1}^{\perp} \cap\left(V_{2}^{1}\right)^{\perp}\right) \\
& =\left(V_{2} \cap V_{1}^{\perp}\right) \cap\left(V_{2}^{1}\right)^{\perp}=V_{2}^{1} \cap\left(V_{2}^{1}\right)^{\perp}=0 .
\end{aligned}
$$

q.e.d.

Proposition 4.8. Suppose $\pi_{1}, \ldots, \pi_{k}$ are Hermitian projections of $\mathbb{C}^{n}$ and $\operatorname{Im} \pi_{j} \cap \operatorname{Im} \pi_{j-1}^{\perp}=0$ for all $2 \leq j \leq k$. Let $n_{j}=$ the rank of $\pi_{j}$. Then

1) $n_{1} \geq n_{2} \geq \cdots \geq n_{k}$,

2) $\operatorname{Ker}\left(\pi_{k}^{\perp} \cdots \pi_{1}^{\perp}\right)=\operatorname{Im} \pi_{1}$,

3) $\operatorname{dim}\left(\operatorname{Im}\left(\pi_{k}^{\perp} \cdots \pi_{1}^{\perp}\right)\right)=n-n_{1}$.

Proof. Denote $V_{j}=\operatorname{Im} \pi_{j}, 1 \leq j \leq k$. The kernel of $\pi_{j}^{\perp}: V_{j-1}^{\perp} \rightarrow V_{j}^{\perp}$ is $V_{j} \cap V_{j-1}^{\perp}=0$. So $\pi_{j}^{\perp}$ is injective on $V_{j-1}^{\perp}$. q.e.d.

Definition 4.9. Suppose $\phi \in \mathcal{S}_{r}\left(S^{2}, G L(n)\right)$ has pole data $(z, k)$. A factorization of $\phi$ is called minimal if

$$
\phi=\left(\frac{\lambda-\bar{z}}{\lambda-z}\right)^{k-l} g_{z, \pi_{l}} \cdots g_{z, \pi_{1}}
$$

with $\pi_{j} \neq 0$, I, and $\operatorname{Im} \pi_{j} \cap \operatorname{Im} \pi_{j-1}^{\perp}=0$ for $j=2, \cdots l$.

Theorem 4.10. If $\phi \in \mathcal{S}_{r}\left(S^{2}, G L(n)\right)$ has pole data $(z, k)$, then $\phi$ has a unique minimal factorization.

Proof. By Uhlenbeck's factorization Theorem 4.1, we can factor

$$
\phi_{k}=g_{z, \pi_{k}} \cdots g_{z, \pi_{1}} .
$$

We first prove the existence of minimal factorization by induction on $k$. For $k=1,2$, the theorem is true. Suppose the theorem is true for $k-1$. Induction hypothesis implies that

$$
g_{z, \pi_{k-1}} \cdots g_{z, \pi_{1}}=\left(\frac{\lambda-\bar{z}}{\lambda-z}\right)^{m} g_{z, \tau_{k-1-m}} \cdots g_{z, \tau_{1}}
$$

so that the right hand side is a minimal factorization. If $m \geq 1$, then by induction hypothesis $g_{z, \pi_{k}} g_{z, \tau_{k-1-m}} \cdots g_{z, \tau_{1}}$ has a minimal factorization. So does $\phi_{k}$. If $m=0$, then there are two cases:

1) If $\operatorname{Im} \pi_{k} \cap \operatorname{Im} \tau_{k-1}^{\perp}=0$, then $g_{z, \pi_{k}} g_{z, \tau_{k-1}} \cdots g_{z, \tau_{1}}$ is a minimal factorization for $\phi_{k}$. 
2) If $V:=\operatorname{Im} \pi_{k} \cap \operatorname{Im} \tau_{k-1}^{\perp} \neq 0$, then by Lemma 4.7 (2) we can write

$$
g_{z, \pi_{k}} g_{z, \tau_{k-1}}=g_{z, \tilde{\pi}_{k}} g_{z, \tilde{\tau}_{k-1}}
$$

such that $\operatorname{Im} \tilde{\pi}_{k} \cap \operatorname{Im} \tilde{\tau}_{k-1}^{\perp}=0$, where $\operatorname{Im} \tilde{\pi}_{k}=\operatorname{Im} \pi_{k} \cap V^{\perp}$, and $\operatorname{Im} \tilde{\tau}_{k-1}=\operatorname{Im} \tau_{k-1} \oplus V$. Since $V \neq 0, \operatorname{rk}\left(\tilde{\pi}_{k}\right)<\operatorname{rk}\left(\pi_{k}\right)$. By induction hypothesis, $g_{z, \tilde{\tau}_{k-1}} g_{z, \tau_{k-2}} \cdots g_{z, \tau_{1}}$ has a minimal factorization

$$
g_{z, \hat{\tau}_{k-1}} \cdots g_{z, \hat{\tau}_{1}} \text {. }
$$

If $\operatorname{Im} \tilde{\pi}_{k} \cap \operatorname{Im} \hat{\tau}_{k-1}^{\perp} \neq 0$, then we use Lemma 4.7 again to reduce the rank of $\tilde{\pi}_{k}$. So after finitely many times, we can obtain a minimal factorization of $\phi_{k}$.

Next we use induction on $k$ to prove the uniqueness of minimal factorization. The case $k=1$ is obvious. Suppose all $\phi$ with pole data $(z, k)$ and $k<K$ have unique minimal factorizations. Consider two minimal factorizations

$$
\left(\frac{\lambda-\bar{z}}{\lambda-z}\right)^{K-l} g_{z, \pi_{l}} \cdots g_{z, \pi_{1}}=\left(\frac{\lambda-\bar{z}}{\lambda-z}\right)^{K-m} g_{z, \tau_{m}} \cdots g_{z, \tau_{1}} .
$$

Compare the coefficients of $\frac{1}{(\lambda-z)^{K}}$ to get

$$
(z-\bar{z})^{K-l} \pi_{l}^{\perp} \cdots \pi_{1}^{\perp}=(z-\bar{z})^{K-m} \tau_{m}^{\perp} \cdots \tau_{1}^{\perp} .
$$

By Proposition 4.8 (2), the kernels of the left hand side and the right hand side operators are $\operatorname{Im} \pi_{1}$ and $\operatorname{Im} \tau_{1}$ respectively. Hence $\pi_{1}=\tau_{1}$. Then induction hypothesis gives the uniqueness.

\section{Ward solitons with pole data $(z, k)$}

Ward noted that the limit of extended 2-soliton solutions with poles at $i+\epsilon$ and $i-\epsilon$ as $\epsilon \rightarrow 0$ gives time dependent Ward maps, and there are 2 -solitons with non-trivial scattering (cf. $[\mathbf{1 4}, \mathbf{6}, \mathbf{8}]$ ). In this section, we use a systematic limiting method and algebraic BTs to construct extended Ward $k$-solitons with pole data $(z, k)$.

First we give the Example of Ward:

Example 5.1. Ward 2-solitons with non-trivial scattering.

Let $\alpha \in \mathbb{C} \backslash \mathbb{R}$, and $f, g$ two rational functions on $\mathbb{C}$. Choose $z_{1}=\alpha+\epsilon$, $z_{2}=\alpha-\epsilon, f_{1}=f+\epsilon g$, and $f_{2}=f-\epsilon g$ in Example 3.2. Expand the formula for $\tilde{v}_{2}$ given by (3.4) in $\epsilon$ to see

$$
\tilde{v}_{2}=C_{1}\left(\begin{array}{c}
1 \\
f(w)
\end{array}\right)+C_{2}\left(\begin{array}{c}
f(w) \\
-1
\end{array}\right)+O(\epsilon)
$$

where $w=x+\alpha u+\alpha^{-1} v$, and

$$
C_{1}=\left(1+|f(w)|^{2}\right), \quad C_{2}=(\bar{\alpha}-\alpha)\left(\left(u-\alpha^{-2} v\right) f^{\prime}(w)+g(w)\right) .
$$


Let $\hat{\pi}_{2}$ denote the projection onto the complex line spanned by

$$
\hat{v}_{2}=C_{1}\left(\begin{array}{c}
1 \\
f(w)
\end{array}\right)+C_{2}\left(\begin{array}{c}
f(w) \\
-1
\end{array}\right) .
$$

Then as $\epsilon \rightarrow 0, \tilde{\pi}_{2}$ tends to $\hat{\pi}_{2}$, the extended solution $\tilde{\psi}$ tends to

$$
\hat{\psi}=g_{\alpha, \hat{\pi}_{2}} g_{\alpha, \pi_{1}}
$$

and the Ward 2-soliton tends to

$$
\hat{J}=\bar{b}\left(\pi_{1}+b \pi_{1}^{\perp}\right)\left(\hat{\pi}_{2}+b \hat{\pi}_{2}^{\perp}\right),
$$

where $b=\alpha / \bar{\alpha}$. In particular, if $\alpha=i$, then we get an extended solution

$$
\hat{\psi}_{2}=g_{i, \hat{\pi}_{2}} g_{i, \pi_{1}}
$$

where $\pi_{1}(x, y, t)$ is the projection onto $\mathbb{C}\left(\begin{array}{c}1 \\ f(w)\end{array}\right)$ and $\hat{\pi}_{2}$ the projection onto the complex line spanned by

$$
\hat{v}_{2}=\left(1+|f(w)|^{2}\right)\left(\begin{array}{c}
1 \\
f(w)
\end{array}\right)-2 i\left(t f^{\prime}(w)+g(w)\right)\left(\begin{array}{c}
\overline{f(w)} \\
-1
\end{array}\right)
$$

and $w=x+i y$. The limiting Ward map $\hat{J}=-\left(\pi_{1}-\pi_{1}^{\perp}\right)\left(\hat{\pi}_{2}-\hat{\pi}_{2}^{\perp}\right)$ is a 2 -soliton with non-trivial scattering, and the extended solution $\hat{\psi}$ has a double pole at $\lambda=i$.

Using similar limiting method, Ioannidou constructed extended Ward 3 -solitons with a triple pole at $\lambda=i$ (see [6]).

Below we apply algebraic BTs and an order $k$ limiting method to construct $k$-solitons, whose extended solutions have pole data $(z, k)$ for any $z \in \mathbb{C} \backslash \mathbb{R}$ and $k \geq 2$. To present this method more clearly, we work on the $S U(2)$ case first. At the end of this section, we will briefly explain how to generalize this method to the $U(n)$ case.

Let $z \in \mathbb{C} \backslash \mathbb{R}$ be a constant, and $\left\{a_{j}(w)\right\}_{j=0}^{\infty}$ a sequence of rational functions in one complex variable. Assume that $a_{0}(w)$ is not a constant function. Let $a_{j}^{(i)}(w)$ denote the $i$-th derivative of $a_{j}$ with respect to $w$. For any $\epsilon \in \mathbb{C}$ with $|\epsilon|$ small, let

$$
\begin{aligned}
w & =x+z u+z^{-1} v, \\
w_{\epsilon} & =x+(z+\epsilon) u+(z+\epsilon)^{-1} v,
\end{aligned}
$$

and

$$
f_{k, \epsilon}=\sum_{j=0}^{k} a_{j}\left(w_{\epsilon}\right) \epsilon^{j} .
$$

A direct computation gives the following Taylor expansions in $\epsilon$ : 
1)

$$
\begin{aligned}
w_{\epsilon}-w & =\epsilon u+\left((z+\epsilon)^{-1}-z^{-1}\right) v \\
& =\left(u-z^{-2} v\right) \epsilon+\sum_{l=2}^{\infty} z^{-l-1} v(-\epsilon)^{l} .
\end{aligned}
$$

2)

$$
a_{j}\left(w_{\epsilon}\right)=\sum_{l=0}^{\infty} \frac{a_{j}^{(l)}(w)}{l !}\left(w_{\epsilon}-w\right)^{l}:=\sum_{l=0}^{\infty} b_{j, l} \epsilon^{l},
$$

where $b_{j, l}=b_{j, l}(x, u, v)$ can be computed directly:

$$
\left\{\begin{aligned}
b_{j, 0}= & a_{j}(w), \\
b_{j, 1}= & \left(u-z^{-2} v\right) a_{j}^{\prime}(w) \\
b_{j, 2}= & \frac{\left(u-z^{-2} v\right)^{2}}{2} a_{j}^{\prime \prime}(w)+\left(z^{-3} v\right) a_{j}^{\prime}(w), \\
b_{j, 3}= & \frac{\left(u-z^{-2} v\right)^{3}}{3 !} a_{j}^{\prime \prime \prime}(w)+\left(u-z^{-2} v\right)\left(z^{-3} v\right) a_{j}^{\prime \prime}(w)-\left(z^{-4} v\right) a_{j}^{\prime}(w), \\
b_{j, 4}= & \frac{\left(u-z^{-2} v\right)^{4}}{{ }^{4 !}} a_{j}^{(4)}(w)+\frac{a_{j}^{\prime \prime \prime}(w)}{2}\left(u-z^{-2} v\right)^{2}\left(z^{-3} v\right) \\
& +\frac{a_{j}^{\prime \prime}(w)}{2}\left(z^{-6} v^{2}-2\left(u-z^{-2} v\right)\left(z^{-4} v\right)\right)+\left(z^{-5} v\right) a_{j}^{\prime}(w), \\
\ldots &
\end{aligned}\right.
$$

3)

$$
\begin{aligned}
f_{k, \epsilon} & =\sum_{j=0}^{k} a_{j}\left(w_{\epsilon}\right) \epsilon^{j}=\sum_{j=0}^{k} \sum_{l=0}^{\infty} b_{j, l} \epsilon^{j+l} \\
& :=c_{0}+c_{1} \epsilon+\cdots+c_{k} \epsilon^{k}+O\left(\epsilon^{k+1}\right),
\end{aligned}
$$

where $c_{l}=c_{l}(x, u, v)=\sum_{j=0}^{l} b_{j, l-j}$ are given below:

$$
\left\{\begin{aligned}
c_{0}= & a_{0}(w), \\
c_{1}= & \left(u-z^{-2} v\right) a_{0}^{\prime}(w)+a_{1}(w), \\
c_{2}= & \frac{\left(u-z^{-2} v\right)^{2}}{2} a_{0}^{\prime \prime}(w)+\left(z^{-3} v\right) a_{0}^{\prime}(w)+\left(u-z^{-2} v\right) a_{1}^{\prime}(w)+a_{2}(w), \\
c_{3}= & \frac{\left(u-z^{-2} v\right)^{3}}{3 !} a_{0}^{\prime \prime \prime}(w)+\left(u-z^{-2} v\right)\left(z^{-3} v\right) a_{0}^{\prime \prime}(w)-\left(z^{-4} v\right) a_{0}^{\prime}(w) \\
& +\frac{\left(u-z^{-2} v\right)^{2}}{2} a_{1}^{\prime \prime}(w)+\left(z^{-3} v\right) a_{1}^{\prime}(w)+\left(u-z^{-2} v\right) a_{2}^{\prime}(w)+a_{3}(w), \\
c_{4}= & \frac{\left(u-z^{-2} v\right)^{4}}{4 !} a_{0}^{(4)}(w)+\frac{a_{0}^{\prime \prime \prime}(w)}{2}\left(u-z^{-2} v\right)^{2}\left(z^{-3} v\right) \\
& +\frac{a_{0}^{\prime \prime}(w)}{2}\left(z^{-6} v^{2}-2\left(u-z^{-2} v\right)\left(z^{-4} v\right)\right)+\left(z^{-5} v\right) a_{0}^{\prime}(w) \\
& +\frac{\left(u-z^{-2} v\right)^{3}}{3 !} a_{1}^{\prime \prime \prime}(w)+\left(u-z^{-2} v\right)\left(z^{-3} v\right) a_{1}^{\prime \prime}(w)-\left(z^{-4} v\right) a_{1}^{\prime}(w) \\
& +\frac{\left(u-z^{-2} v\right)^{2}}{2} a_{2}^{\prime \prime}(w)+\left(z^{-3} v\right) a_{2}^{\prime}(w)+\left(u-z^{-2} v\right) a_{3}^{\prime}(w)+a_{4}(w), \\
\cdots &
\end{aligned}\right.
$$

From the above computation, we see that $c_{j}$ 's are rational functions in $x, u, v$, and hence are rational in $x, y$ and $t$. 
For $k \geq 1$, let

$$
v_{k, \epsilon}=\left(\begin{array}{c}
1 \\
f_{k-1, \epsilon}
\end{array}\right)
$$

$\pi_{k, \epsilon}$ the Hermitian projection of $\mathbb{C}^{2}$ onto $\mathbb{C} v_{k, \epsilon}$. Define $\psi_{k, \epsilon}$ and $\hat{\psi}_{k}$ by induction as follows:

$$
\begin{aligned}
& \psi_{1, \epsilon}=g_{z+\epsilon, \pi_{1, \epsilon}}, \quad \text { and } \quad \hat{\psi}_{1}=\lim _{\epsilon \rightarrow 0} \psi_{1, \epsilon}, \\
& \psi_{k, \epsilon}=g_{z+\epsilon, \pi_{k, \epsilon}} * \hat{\psi}_{k-1}, \quad \text { and } \quad \hat{\psi}_{k}=\lim _{\epsilon \rightarrow 0} \psi_{k, \epsilon} .
\end{aligned}
$$

Let

$$
\tilde{v}_{k, \epsilon}=\hat{\psi}_{k-1}(z+\epsilon)\left(v_{k, \epsilon}\right) .
$$

By Theorem 3.1,

$$
\psi_{k, \epsilon}=g_{z+\epsilon, \tilde{\pi}_{k, \epsilon}} \hat{\psi}_{k-1},
$$

where $\tilde{\pi}_{k, \epsilon}$ is the projection onto $\mathbb{C} \tilde{v}_{k, \epsilon}$.

Theorem 5.2. Let $a_{0}, a_{1}, \ldots$ be a sequence of rational functions from $\mathbb{C}$ to $\mathbb{C}$, and let $v_{k, \epsilon}, \pi_{k, \epsilon}, \psi_{k, \epsilon}, \hat{\psi}_{k}$ and $\tilde{v}_{k, \epsilon}$ be defined as above. Then we have

(1) $\tilde{v}_{k, \epsilon}=\hat{v}_{k}+\epsilon y_{k, 1}+\epsilon^{2} y_{k, 2}+\cdots$, where

$$
\begin{gathered}
\hat{v}_{k}=\left(\begin{array}{c}
1 \\
c_{0}
\end{array}\right)+\sum_{j=1}^{k-1}(z-\bar{z})^{j} P_{k-1, j}\left(\begin{array}{c}
0 \\
c_{j}
\end{array}\right), \\
P_{l, j}=\sum_{l \geq i_{1}>\cdots>i_{j} \geq 1} \hat{\pi}_{i_{1}}^{\perp} \cdots \hat{\pi}_{i_{j}}^{\perp} .
\end{gathered}
$$

Moreover, all entries of $\hat{v}_{k}$ are rational functions in $x, y$ and $t$.

(2) $\hat{\psi}_{k}=g_{z, \hat{\pi}_{k}} \cdots g_{z, \hat{\pi}_{1}}$ is a minimal factorization and an extended Ward map with only a pole at $\lambda=z$ of multiplicity $k$, where $\hat{\pi}_{k}$ is the Hermitian projection of $\mathbb{C}^{2}$ onto $\mathbb{C} \hat{v}_{k}$. Moreover, $\hat{\pi}_{k}$ is smooth, and for each fixed $t, \lim _{|x|^{2}+|y|^{2} \rightarrow \infty} \hat{\pi}_{k}(x, y, t)$ exists.

(3) The Ward map associated to $\hat{\psi}_{k}$,

$$
J_{k}=\frac{1}{|z|^{k}}\left(\bar{z} \hat{\pi}_{1}+z \hat{\pi}_{1}^{\perp}\right)\left(\bar{z} \hat{\pi}_{2}+z \hat{\pi}_{2}^{\perp}\right) \cdots\left(\bar{z} \hat{\pi}_{k}+z \hat{\pi}_{k}^{\perp}\right),
$$

is smooth and satisfies the boundary condition (1.8), and all entries of $J_{k}$ are rational functions in $x, y$ and $t$.

Proof. We prove the theorem by induction on $k$. For $k=1$, the theorem is clearly true. Suppose the theorem is true for $k$. We will prove that (1)-(3) hold for $k+1$. 
(1) By Theorem 3.1 and induction hypothesis, we have

$$
\begin{aligned}
& \tilde{v}_{k+1, \epsilon}=\hat{\psi}_{k}(z+\epsilon) v_{k+1, \epsilon} \\
& =\left(\mathrm{I}+\frac{z-\bar{z}}{\epsilon} \hat{\pi}_{k}^{\perp}\right) \hat{\psi}_{k-1}(z+\epsilon)\left(\begin{array}{c}
1 \\
f_{k, \epsilon}
\end{array}\right) \\
& =\left(\mathrm{I}+\frac{z-\bar{z}}{\epsilon} \hat{\pi}_{k}^{\perp}\right) \hat{\psi}_{k-1}(z+\epsilon)\left(\begin{array}{c}
1 \\
f_{k-1, \epsilon}+a_{k}\left(w_{\epsilon}\right) \epsilon^{k}
\end{array}\right) \\
& =\left(\mathrm{I}+\frac{z-\bar{z}}{\epsilon} \hat{\pi}_{k}^{\perp}\right)\left(\hat{\psi}_{k-1}(z+\epsilon)\left(\begin{array}{c}
1 \\
f_{k-1, \epsilon}
\end{array}\right)+\hat{\psi}_{k-1}(z+\epsilon)\left(\begin{array}{c}
0 \\
a_{k}\left(w_{\epsilon}\right) \epsilon^{k}
\end{array}\right)\right) \\
& =\left(\mathrm{I}+\frac{z-\bar{z}}{\epsilon} \hat{\pi}_{k}^{\perp}\right)\left(\hat{v}_{k}+\epsilon y_{k, 1}+\epsilon(z-\bar{z})^{k-1} P_{k-1, k-1}\left(\begin{array}{c}
0 \\
a_{k}(w)
\end{array}\right)+O\left(\epsilon^{2}\right)\right) \\
& =\hat{v}_{k}+(z-\bar{z}) \hat{\pi}_{k}^{\perp}\left(y_{k, 1}+(z-\bar{z})^{k-1} P_{k-1, k-1}\left(\begin{array}{c}
0 \\
a_{k}(w)
\end{array}\right)\right)+O(\epsilon) .
\end{aligned}
$$

In the last step we have used $\hat{v}_{k} \in \operatorname{Im} \hat{\pi}_{k}$. Thus all terms of negative powers of $\epsilon$ vanish in the Laurent series expansion of $\tilde{v}_{k+1, \epsilon}$ in $\epsilon$.

The Laurent series expansion of $\hat{\psi}_{k}(z+\epsilon)$ in $\epsilon$ is

$$
\begin{aligned}
\hat{\psi}_{k}(z+\epsilon) & =\left(\mathrm{I}+\frac{z-\bar{z}}{\epsilon} \hat{\pi}_{k}^{\perp}\right) \cdots\left(\mathrm{I}+\frac{z-\bar{z}}{\epsilon} \hat{\pi}_{1}^{\perp}\right) \\
& =\mathrm{I}+\frac{z-\bar{z}}{\epsilon} P_{k, 1}+\cdots+\frac{(z-\bar{z})^{k}}{\epsilon^{k}} P_{k, k} .
\end{aligned}
$$

Substituting (5.5) to $\tilde{v}_{k+1, \epsilon}$ and using the fact that the Laurent series expansion of $\tilde{v}_{k+1, \epsilon}$ has no $\epsilon^{-j}$ terms with $j>0$, we have

$$
\begin{aligned}
\tilde{v}_{k+1, \epsilon} & =\hat{\psi}_{k}(z+\epsilon) v_{k+1, \epsilon}=\hat{\psi}_{k}(z+\epsilon)\left(\begin{array}{c}
1 \\
f_{k, \epsilon}
\end{array}\right) \\
& =\hat{\psi}_{k}(z+\epsilon)\left(\left(\begin{array}{c}
1 \\
c_{0}
\end{array}\right)+\epsilon\left(\begin{array}{c}
0 \\
c_{1}
\end{array}\right)+\cdots+\epsilon^{k}\left(\begin{array}{c}
0 \\
c_{k}
\end{array}\right)+O\left(\epsilon^{k+1}\right)\right) \\
& =\left(\begin{array}{c}
1 \\
c_{0}
\end{array}\right)+(z-\bar{z}) P_{k, 1}\left(\begin{array}{c}
0 \\
c_{1}
\end{array}\right)+\cdots+(z-\bar{z})^{k} P_{k, k}\left(\begin{array}{c}
0 \\
c_{k}
\end{array}\right)+O(\epsilon) .
\end{aligned}
$$

Therefore we obtain

$$
\hat{v}_{k+1}=\left(\begin{array}{c}
1 \\
c_{0}
\end{array}\right)+\sum_{j=1}^{k}(z-\bar{z})^{j} P_{k, j}\left(\begin{array}{c}
0 \\
c_{j}
\end{array}\right) .
$$

By induction hypothesis, $\hat{\pi}_{1}, \ldots, \hat{\pi}_{k}$ are smooth, and all of their entries are rational in $x, y$ and $t$. Thus $P_{k, 1}, \ldots, P_{k, k}$ have the same analytic properties as $\pi_{j}$ 's. Together with the analytic properties of $c_{j}$ 's, we see that all entries of $\hat{v}_{k+1}$ are rational in $x, y$ and $t$.

(2) By (1), we have

$$
\lim _{\epsilon \rightarrow 0} \tilde{\pi}_{k+1, \epsilon}=\hat{\pi}_{k+1}
$$


where $\hat{\pi}_{k+1}$ is the projection onto $\mathbb{C} \hat{v}_{k+1}$. Since all entries of $\hat{v}_{k+1}$ are rational in $x, y$ and $t, \hat{\pi}_{k+1}$ is smooth, and for each fixed $t$,

$$
\lim _{|x|^{2}+|y|^{2} \rightarrow \infty} \hat{\pi}_{k+1}(x, y, t)
$$

exists.

Next we claim that $\hat{\psi}_{k+1}$ is an extended Ward map with a pole at $\lambda=z$ of multiplicity $k+1$. To see this, first note that

$$
\begin{aligned}
\hat{\psi}_{k+1} & =\lim _{\epsilon \rightarrow 0} g_{z+\epsilon, \pi_{k+1, \epsilon}} * \hat{\psi}_{k}=\lim _{\epsilon \rightarrow 0} g_{z+\epsilon, \tilde{\pi}_{k+1, \epsilon}} \hat{\psi}_{k} \\
& =g_{z, \hat{\pi}_{k+1}} g_{z, \hat{\pi}_{k}} \cdots g_{z, \hat{\pi}_{1}}=\left(\mathrm{I}+\frac{z-\bar{z}}{\lambda-z} \hat{\pi}_{k+1}^{\perp}\right) \cdots\left(\mathrm{I}+\frac{z-\bar{z}}{\lambda-z} \hat{\pi}_{1}^{\perp}\right) \\
& =\mathrm{I}+\sum_{j=1}^{k+1} \frac{(z-\bar{z})^{j}}{(\lambda-z)^{j}} P_{k+1, j} .
\end{aligned}
$$

By Theorem 3.1, $g_{z+\epsilon, \pi_{k+1, \epsilon}} * \hat{\psi}_{k}$ is an extended Ward map for small $|\epsilon|>0$. By continuity, so is $\hat{\psi}_{k+1}$. The coefficient of $(\lambda-z)^{-k-1}$ of $\hat{\psi}_{k+1}$ is $(z-\bar{z})^{k+1} P_{k+1, k+1}$. To show that $\hat{\psi}_{k+1}$ has a pole at $\lambda=z$ of multiplicity $k+1$, it suffices to show that $P_{k+1, k+1}=\hat{\pi}_{k+1}^{\perp} \hat{\pi}_{k}^{\perp} \cdots \hat{\pi}_{1}^{\perp} \neq 0$. For this purpose, we write

$$
P_{k, j}=P_{k-1, j}+\hat{\pi}_{k}^{\perp} P_{k-1, j-1} .
$$

So (5.6) for $k+1$ can be written as

$$
\begin{aligned}
& \hat{v}_{k+1} \\
& =\left(\begin{array}{c}
1 \\
c_{0}
\end{array}\right)+\sum_{j=1}^{k-1}(z-\bar{z})^{j} P_{k-1, j}\left(\begin{array}{c}
0 \\
c_{j}
\end{array}\right)+\hat{\pi}_{k}^{\perp} \sum_{j=0}^{k-1}(z-\bar{z})^{j+1} P_{k-1, j}\left(\begin{array}{c}
0 \\
c_{j+1}
\end{array}\right) \\
& =\hat{v}_{k}+(z-\bar{z}) \hat{\pi}_{k}^{\perp} \sum_{j=0}^{k-1}(z-\bar{z})^{j} P_{k-1, j}\left(\begin{array}{c}
0 \\
c_{j+1}
\end{array}\right) .
\end{aligned}
$$

By the induction hypothesis, $\hat{\psi}_{k}=g_{z, \hat{\pi}_{k}} \cdots g_{z, \hat{\pi}_{1}}$ is a minimal factorization. So $\left(\operatorname{Im} \pi_{j+1}\right) \cap\left(\operatorname{Im} \pi_{j}^{\perp}\right)=0$ for $1 \leq j \leq k-1$. By induction hypothesis $\hat{v}_{k} \neq 0$. The above formula implies that $\operatorname{Im} \hat{\pi}_{k+1} \cap \operatorname{Im} \hat{\pi}_{k}^{\perp}=0$. Hence $\hat{\psi}_{k+1}$ is a minimal factorization. By Proposition 4.8, $\hat{\pi}_{k+1}^{\perp} \hat{\pi}_{k}^{\perp} \cdots \hat{\pi}_{1}^{\perp} \neq 0$. Thus (2) holds for $k+1$.

(3) The expression for $J_{k+1}=\hat{\psi}_{k+1}(0)^{-1}$ is straightforward. Since all $\pi_{j}$ 's are smooth, and for each fixed $t, \lim _{|x|^{2}+|y|^{2} \rightarrow \infty} \pi_{j}(x, y, t)$ exists, $J_{k+1}$ is also smooth and satisfies the boundary condition (1.8). The entries of $J_{k+1}$ are rational in $x, y$ and $t$ because all $\pi_{j}$ 's have this property. q.e.d.

We give some explicit formulas for $\hat{v}_{k}$ with $k$ small: 
1) $\hat{v}_{1}=\left(\begin{array}{c}1 \\ c_{0}\end{array}\right)$.

2) $\hat{v}_{2}=\hat{v}_{1}+(z-\bar{z}) \hat{\pi}_{1}^{\perp}\left(\begin{array}{c}0 \\ c_{1}\end{array}\right)$. The corresponding Ward map is with the one in Example 5.1 if $z=\alpha$ and $\left(a_{0}, a_{1}\right)=(f, g)$.

3) $\hat{v}_{3}=\hat{v}_{2}+(z-\bar{z}) \hat{\pi}_{2}^{\perp}\left(\left(\begin{array}{c}0 \\ c_{1}\end{array}\right)+(z-\bar{z}) \hat{\pi}_{1}^{\perp}\left(\begin{array}{c}0 \\ c_{2}\end{array}\right)\right)$.

4)

$$
\begin{aligned}
& \hat{v}_{4}-\hat{v}_{3} \\
& =(z-\bar{z}) \hat{\pi}_{3}^{\perp}\left(\left(\begin{array}{c}
0 \\
c_{1}
\end{array}\right)+(z-\bar{z})\left(\hat{\pi}_{2}^{\perp}+\hat{\pi}_{1}^{\perp}\right)\left(\begin{array}{c}
0 \\
c_{2}
\end{array}\right)+(z-\bar{z})^{2} \hat{\pi}_{2}^{\perp} \hat{\pi}_{1}^{\perp}\left(\begin{array}{c}
0 \\
c_{3}
\end{array}\right)\right) .
\end{aligned}
$$

We briefly explain how to construct Ward solitons with pole data $(z, k)$ for the $U(n)$ case next. Choose a sequence of rational maps $a_{j}$ : $\mathbb{C} \rightarrow \mathbb{C}^{n}, j=0,1,2, \cdots$. Let $\pi_{j, \epsilon}$ be the Hermitian projection of $\mathbb{C}^{n}$ onto $\mathbb{C} \sum_{l=0}^{j-1} a_{l}\left(w_{\epsilon}\right) \epsilon^{l}, w_{\epsilon}=x+(z+\epsilon) u+(z+\epsilon)^{-1} v$, for $1 \leq j \leq k$. Then the same computation and proof as in the $S U(2)$ case imply that $\hat{\psi}_{k}=g_{z, \hat{\pi}_{k}} g_{z, \hat{\pi}_{k-1}} \cdots g_{z, \hat{\pi}_{1}}$ is a minimal factorization and is an extended Ward map with pole data $(z, k)$.

Note that all $\hat{\pi}_{j}$ 's are of rank one in the above construction. But the same limiting method also produces extended Ward solitons of the form

$$
g_{z, \pi_{k}} \cdots g_{z, \pi_{1}}
$$

with $\operatorname{rk}\left(\pi_{1}\right) \geq \cdots \geq \operatorname{rk}\left(\pi_{k}\right)$. To see this, let $n-1 \geq n_{1} \geq \cdots \geq n_{k} \geq 1$ be integers, and $a_{i 1}, \ldots, a_{i n_{i}} \mathbb{C}^{n}$-valued rational maps on $\mathbb{C}$ for $1 \leq i \leq k$. Suppose

$$
a_{11} \wedge \cdots \wedge a_{1 n_{1}} \neq 0
$$

generically, and let $\pi_{1}(w)$ denote the projection of $\mathbb{C}^{n}$ onto the linear span of $a_{11}(w), \ldots, a_{1 n_{1}}(w)$. Let $w=x+z u+z^{-1} v$, and $w_{\epsilon}=x+(z+$ $\epsilon) u+(z+\epsilon)^{-1} v$. Then

$$
\begin{aligned}
\hat{v}_{2 i} & :=\lim _{\epsilon \rightarrow 0} g_{z, \pi_{1}}(z+\epsilon)\left(a_{1 i}\left(w_{\epsilon}\right)+\epsilon a_{2 i}\left(w_{\epsilon}\right)\right) \\
& =a_{1 i}(w)+(z-\bar{z}) \pi_{1}^{\perp}\left(\left(u-z^{-2} v\right) a_{1 i}^{\prime}(w)+a_{2 i}(w)\right), \quad 1 \leq i \leq n_{2} .
\end{aligned}
$$

Let $\pi_{2}$ denote the projection onto the linear span of $\hat{v}_{21}(w), \ldots, \hat{v}_{2 n_{2}}(w)$. Then $g_{z, \pi_{2}} g_{z, \pi_{1}}$ is an extended solution, and $\operatorname{rk}\left(\pi_{2}\right)=n_{2}$. It is easy to see that $\operatorname{Im} \pi_{2} \cap \operatorname{Im} \pi_{1}^{\perp}=0$. Hence $g_{z, \pi_{2}} g_{z, \pi_{1}}$ is a minimal factorization. Similar computations give the construction of extended Ward maps with pole data $(z, k)$ and arbitrary rank data $\left(n_{1}, \ldots, n_{k}\right)$.

\section{Ward solitons with general pole data}

We associate to each extended Ward map with pole data $(z, k)$ a generalized algebraic BT. Using these generalized BTs, we construct extended Ward maps that have general pole data $\left(z_{1}, \ldots, z_{k}, n_{1}, \ldots, n_{k}\right)$. 
We first give a more general algebraic BT (Theorem 3.1 is the case when $k=1)$ :

Theorem 6.1. Let $\phi$ be an extended Ward map with pole data $(z, k)$, and $\psi$ an extended Ward map that is holomorphic and non-degenerate at $\lambda=z, \bar{z}$. Then there exist unique $\tilde{\phi}$ and $\tilde{\psi}$ such that $\tilde{\phi} \psi=\tilde{\psi} \phi$, where $\tilde{\phi}$ has pole data $(z, k)$, and $\tilde{\psi}$ is holomorphic and non-degenerate at $\lambda=z, \bar{z}$. Moreover,

$$
\psi_{k}=\tilde{\phi} \psi=\tilde{\psi} \phi
$$

is a new extended Ward map and $\tilde{\phi}, \tilde{\psi}$ are constructed algebraically.

Proof. It follows from Theorem 4.1 that we can factor $\phi$ as product of $k$ simple elements.

$$
\phi=g_{z, \pi_{k}} \cdots g_{z, \pi_{1}} .
$$

Let $\tilde{\pi}_{1}$ be the projection onto $\psi(z) \operatorname{Im} \pi_{1}$, and $\tilde{\psi}_{1}=g_{z, \tilde{\pi}_{1}} \psi g_{z, \pi_{1}}^{-1}$. Residue calculus implies that $\tilde{\psi}_{1}$ is holomorphic at $\lambda=z, \bar{z}$. For $j=2, \ldots, k$, we define $\tilde{\pi}_{j}$ and $\tilde{\psi}_{j}$ recursively by

$$
\operatorname{Im} \tilde{\pi}_{j}=\tilde{\psi}_{j-1}(z) \operatorname{Im} \pi_{j}, \quad \text { and } \quad \tilde{\psi}_{j}=g_{z, \tilde{\pi}_{j}} \tilde{\psi}_{j-1} g_{z, \pi_{j}}^{-1} .
$$

Again $\tilde{\psi}_{j}$ is holomorphic at $\lambda=z, \bar{z}$ for $j=2, \ldots, k$ by residual calculus. Let $\tilde{\phi}=g_{z, \tilde{\pi}_{k}} \cdots g_{z, \tilde{\pi}_{1}}$, and $\tilde{\psi}=\tilde{\psi}_{k}$. By construction, $\tilde{\phi} \psi=\tilde{\psi} \phi$. Next we prove uniqueness. Suppose $\hat{\phi}$ has pole data $(z, k)$ and $\hat{\psi}$ is holomorphic and non-degenerate at $z, \bar{z}$ and $\hat{\phi} \psi=\hat{\psi} \phi$. Then $\phi \psi^{-1}=\tilde{\psi}^{-1} \tilde{\phi}=\hat{\psi}^{-1} \hat{\phi}$. So we have

$$
\hat{\psi} \tilde{\psi}^{-1}=\hat{\phi} \tilde{\phi}^{-1} \text {. }
$$

But the left hand side is holomorphic at $z, \bar{z}$ and the right hand side is holomorphic at $\lambda \in \mathbb{C} \backslash\{z, \bar{z}\}$ and is equal to I at $\lambda=\infty$. Hence it must be the constant identity. This proves $\tilde{\phi}=\hat{\phi}$ and $\tilde{\psi}=\hat{\psi}$.

The same proof of Theorem 3.1 implies that $\psi_{k}=\tilde{\psi} \phi=\tilde{\phi} \psi$ is an extended Ward map.

q.e.d.

We use $\phi * \psi$ to denote the new extended solution $\psi_{k}=\tilde{\phi} \psi$ constructed in the above theorem, and call

$$
\psi \mapsto \phi * \psi
$$

the generalized Bäcklund transformation generated by $\phi$.

The proof of Theorem 6.1 implies that if $\psi$ and $\phi$ are extended Ward maps with pole data $\left(z_{1}, n_{1}\right)$ and $\left(z_{2}, n_{2}\right)$ respectively and $z_{1} \neq z_{2}, \bar{z}_{2}$, then $\phi * \psi=\psi * \phi$. The same argument gives the following corollary:

Corollary 6.2. Let $z_{1}, \ldots, z_{r} \in \mathbb{C} \backslash \mathbb{R}$ such that $z_{i} \neq z_{j}, \bar{z}_{j}$ for all $i \neq$ $j$, and $\phi_{j}$ an extended Ward map with pole data $\left(z_{j}, n_{j}\right)$ for $1 \leq j \leq r$. Let $\sigma$ be a permutation of $\{1, \ldots, k\}$. Then

$$
\phi_{\sigma(1)} *\left(\phi_{\sigma(2)} *\left(\cdots * \phi_{\sigma(r)}\right) \cdots\right)=\phi_{1} *\left(\phi_{2} *\left(\cdots * \phi_{r}\right) \cdots\right) .
$$


Example 6.3. Extended Ward 4-solitons into $S U(2)$ with two double poles.

Choose $z_{1}, z_{2} \in \mathbb{C} \backslash \mathbb{R}$ with $z_{1} \neq z_{2}, \bar{z}_{2}$, and rational functions $a_{0}(w)$, $a_{1}(w)$ and $b_{0}(w), b_{1}(w)$. Let $w_{i}=x+z_{i} u+z_{i}^{-1} v, i=1,2$. By the construction of section 5 , we have two extended Ward solitons

$$
\phi=g_{z_{2}, \pi_{2}} g_{z_{2}, \pi_{1}}, \quad \psi=g_{z_{1}, \tau_{2}} g_{z_{1}, \tau_{1}},
$$

where

$$
\begin{gathered}
\operatorname{Im} \pi_{1}=\mathbb{C} v_{1}=\mathbb{C}\left(\begin{array}{c}
1 \\
b_{0}\left(w_{2}\right)
\end{array}\right), \quad \operatorname{Im} \pi_{2}=\mathbb{C} v_{2}, \\
v_{2}=\left(\begin{array}{c}
1 \\
b_{0}\left(w_{2}\right)
\end{array}\right)+\left(z_{2}-\bar{z}_{2}\right) \pi_{1}^{\perp}\left(\begin{array}{c}
0 \\
\left(u-z_{2}^{-2} v\right) b_{0}^{\prime}\left(w_{2}\right)+b_{1}\left(w_{2}\right)
\end{array}\right) \\
=\left(\begin{array}{c}
1 \\
b_{0}\left(w_{2}\right)
\end{array}\right)-\frac{\left(z_{2}-\bar{z}_{2}\right)\left(\left(u-z_{2}^{-2} v\right) b_{0}^{\prime}\left(w_{2}\right)+b_{1}\left(w_{2}\right)\right)}{1+\left|b_{0}\left(w_{2}\right)\right|^{2}}\left(\begin{array}{c}
\overline{b_{0}\left(w_{2}\right)} \\
-1
\end{array}\right),
\end{gathered}
$$

and

$$
\begin{gathered}
\operatorname{Im} \tau_{1}=\mathbb{C} q_{1}=\mathbb{C}\left(\begin{array}{c}
1 \\
a_{0}\left(w_{1}\right)
\end{array}\right), \quad \operatorname{Im} \tau_{2}=\mathbb{C} q_{2}, \\
q_{2}=\left(\begin{array}{c}
1 \\
a_{0}\left(w_{1}\right)
\end{array}\right)+\left(z_{1}-\bar{z}_{1}\right) \tau_{1}^{\perp}\left(\begin{array}{c}
0 \\
\left(u-z_{1}^{-2} v\right) a_{0}^{\prime}\left(w_{1}\right)+a_{1}\left(w_{1}\right)
\end{array}\right) \\
=\left(\begin{array}{c}
1 \\
a_{0}\left(w_{1}\right)
\end{array}\right)-\frac{\left(z_{1}-\bar{z}_{1}\right)\left(\left(u-z_{1}^{-2} v\right) a_{0}^{\prime}\left(w_{1}\right)+a_{1}\left(w_{1}\right)\right)}{1+\left|a_{0}\left(w_{1}\right)\right|^{2}}\left(\begin{array}{c}
\overline{a_{0}\left(w_{1}\right)} \\
-1
\end{array}\right) .
\end{gathered}
$$

Apply generalized Bäcklund transformation $\phi * \psi$ to get an extended solution with two double poles at $z_{1}, z_{2}$. By Theorem 6.1,

$$
\phi * \psi=g_{z_{2}, \tilde{\pi}_{2}} g_{z_{2}, \tilde{\pi}_{1}} g_{z_{1}, \tau_{2}} g_{z_{1}, \tau_{1}},
$$

where $\operatorname{Im} \tilde{\pi}_{1}=\mathbb{C} \tilde{v}_{1}=\mathbb{C} \psi\left(z_{2}\right) v_{1}$, and $\operatorname{Im} \tilde{\pi}_{2}=\mathbb{C} \tilde{v}_{2}=\mathbb{C} \tilde{\psi}_{1}\left(z_{2}\right) v_{2}$. Compute the following limit,

$$
\left(z_{2}-\bar{z}_{2}\right) \tilde{\psi}_{1}\left(z_{2}\right)=\lim _{\lambda \rightarrow z_{2}}\left(\lambda-\bar{z}_{2}\right) g_{z_{2}, \tilde{\pi}_{1}}(\lambda) \psi(\lambda) g_{z_{2}, \pi_{1}}(\lambda)^{-1},
$$

to see

$$
\tilde{\psi}_{1}\left(z_{2}\right)=\psi\left(z_{2}\right) \pi_{1}+\tilde{\pi}_{1}^{\perp}\left(\psi\left(z_{2}\right)+\left(z_{2}-\bar{z}_{2}\right) \frac{\partial \psi\left(z_{2}\right)}{\partial \lambda} \pi_{1}\right) .
$$

The associated Ward 4-soliton is given by

$$
J_{4}=\frac{1}{\left|z_{1} z_{2}\right|^{2}}\left(\bar{z}_{1} \tau_{1}+z_{1} \tau_{1}^{\perp}\right)\left(\bar{z}_{1} \tau_{2}+z_{1} \tau_{2}^{\perp}\right)\left(\bar{z}_{2} \tilde{\pi}_{1}+z_{2} \tilde{\pi}_{1}^{\perp}\right)\left(\bar{z}_{2} \tilde{\pi}_{2}+z_{2} \tilde{\pi}_{2}^{\perp}\right) .
$$

Let

$$
\mathbb{C}_{ \pm}=\{a \pm i b \mid b>0\}
$$

denote the upper and lower half plane of $\mathbb{C}$. We claim that to construct Ward solitons with general pole data, we may assume all the poles lie in the upper half plane $\mathbb{C}_{+}$. This claim follows from two remarks below: 
(1) A direct computation implies that

$$
\frac{\lambda-z}{\lambda-\bar{z}} g_{z, \pi}(\lambda)=\mathrm{I}+\frac{\bar{z}-z}{\lambda-\bar{z}} \pi=g_{\bar{z}, \pi^{\perp}} .
$$

(2) Let $\psi$ be an extended Ward soliton. By Theorem 4.1, we can factor

$$
\psi=g_{z_{1}, \pi_{1}} \cdots g_{z_{r}, \pi_{r}} .
$$

Suppose $z_{1}, \ldots, z_{k} \in \mathbb{C}_{-}$and the rest of the poles lie in $\mathbb{C}_{+}$. Let

$$
f(\lambda)=\prod_{j=1}^{k} \frac{\lambda-z_{j}}{\lambda-\bar{z}_{j}} .
$$

Then $f \psi$ still satisfies (1.4), and hence is an extended solution. But $f \psi$ has poles at $\bar{z}_{1}, \ldots, \bar{z}_{k}, z_{k+1}, \ldots, z_{r}$, which all lie in $\mathbb{C}_{+}$. The Ward maps corresponding to $\psi$ and $f \psi$ are $J=\psi(0)^{-1}$ and $J_{1}=\psi(0)^{-1} / f(0)$ respectively. Note $f(0)$ is a constant complex number of length 1 . So we do not lose any Ward maps by assuming that all poles lie in $\mathbb{C}_{+}$.

Corollary 6.4. Given distinct $z_{1}, \ldots, z_{r} \in \mathbb{C}_{+}$and positive integers $n_{1}, \ldots, n_{r}$, there is a family of Ward solitons whose extended solutions have pole data $\left(z_{1}, \ldots, z_{r}, n_{1}, \ldots, n_{r}\right)$.

Proof. Let $\phi_{z_{j}, n_{j}}=g_{z_{j}, \pi_{n_{j}}} \cdots g_{z_{j}, \pi_{1}}$ be an extended Ward soliton with pole data $\left(z_{j}, n_{j}\right)$ constructed in Section 5. Apply Theorem 6.1 repeatedly to $\phi_{z_{1}, n_{1}}$ to get the extended solution

$$
\phi=\phi_{z_{r}, n_{r}} *\left(\cdots *\left(\phi_{z_{2}, n_{2}} * \phi_{z_{1}, n_{1}}\right) \cdots\right) .
$$

Then $\phi$ has pole data $\left(z_{1}, \ldots, z_{r}, n_{1}, \ldots, n_{r}\right)$.

q.e.d.

In the rest of the section, we prove that a general Ward soliton can be constructed by applying generalized Bäcklund transformations to an extended Ward soliton with pole data $(z, k)$.

Theorem 6.5. Suppose $\psi$ is an extended solution of the Ward equation, and $\psi=f_{1} f_{2}$, such that

1) $\lambda \mapsto f_{2}(x, y, t, \lambda)$ is an element of the group $\mathcal{S}_{r}\left(S^{2}, G L(n)\right)$ and has poles only at $z_{1}, \ldots, z_{k}$,

2) $f_{1}$ is holomorphic and non-degenerate at $\lambda=z_{i}$ and $\bar{z}_{i}$ for $1 \leq i \leq$ $k$.

Then $f_{2}$ is also an extended solution of the Ward equation.

Proof. Let $P f=\lambda f_{x}-f_{u}, Q f=\lambda f_{v}-f_{x}$, and $D=\left\{z_{i}, \bar{z}_{i} \mid 1 \leq i \leq k\right\}$. Use $f_{2}=f_{1}^{-1} \psi$ to compute directly to get

$$
A_{2}:=\left(P f_{2}\right) f_{2}^{-1}=P\left(f_{1}^{-1}\right) f_{1}+f_{1}^{-1}(P \psi) \psi^{-1} f_{1} .
$$

Since $\psi$ is an extended solution, $A=(P \psi) \psi^{-1}$ is independent of $\lambda$. Because $f_{1}$ is holomorphic and non-degenerate at points in $D$, the right hand side of $A_{2}$ is holomorphic at points in $D$. But $f_{2}$ is assumed to be 
holomorphic in $\mathbb{C} \backslash D$, so $\left(P f_{2}\right) f_{2}^{-1}$ is holomorphic for $\lambda \in \mathbb{C} \backslash D$. So $A_{2}(x, y, t, \lambda)$ is holomorphic for all $\lambda \in \mathbb{C}$. But $f_{2} \in \mathcal{S}_{r}\left(S^{2}, G L(n)\right)$ implies that $\left(P f_{2}\right) f_{2}^{-1}$ is holomorphic at $\lambda=\infty$. Hence $A_{2}$ is independent of $\lambda$. Similarly, $\left(Q f_{2}\right) f_{2}^{-1}$ is independent of $\lambda$. This proves that $f_{2}$ is an extended solution of the Ward equation.

q.e.d.

Corollary 6.6. Suppose $\psi=\phi_{1} \cdots \phi_{r}$ is an extended Ward soliton such that $\phi_{j}$ has pole data $\left(z_{j}, n_{j}\right)$ and $z_{1}, \ldots, z_{r} \in \mathbb{C}_{+}$are distinct. Then for $1 \leq j \leq r-1$,

1) $\psi_{j}=\phi_{j+1} \ldots \phi_{r}$ is also an extended Ward soliton,

2) there exists a unique extended Ward soliton $\tilde{\phi}_{j}$ with pole data $\left(z_{j}, n_{j}\right)$ so that $\psi_{j-1}=\tilde{\phi}_{j} * \psi_{j}$,

3) $\psi$ can be constructed by applying the generalized algebraic Bäcklund transformations repeatedly to $\phi_{r}$.

Proposition 6.7. If $\psi$ is an extended Ward soliton with pole data $\left(z_{1}, \ldots, z_{r}, n_{1}, \ldots, n_{r}\right)$, then for $1 \leq j \leq r$, there exists a unique extended Ward soliton $\phi_{j}$ with pole data $\left(z_{j}, n_{j}\right)$ so that

$$
\psi=\phi_{1} *\left(\phi_{2} *\left(\cdots * \phi_{r}\right) \cdots\right) .
$$

Proof. By Uhlenbeck's factorization Theorem 4.1 and the permutability Theorem 4.2 , we can factor $\psi$ as

$$
\psi=f_{2} \cdots f_{r} f_{1}=g_{1}\left(g_{2} \cdots g_{r}\right)
$$

such that $f_{j}, g_{j} \in \mathcal{S}_{r}\left(S^{2}, G L(n)\right)$ have pole data $\left(z_{j}, n_{j}\right)$. We prove the proposition by induction on $r$. If $r=1$, the proposition is automatically true. Suppose the proposition is true for $r=n-1$. Then by Theorem 6.5 both $f_{1}$ and $g_{2} \cdots g_{r}$ are extended solutions. By induction hypothesis, there exist extended Ward maps $h_{2}, \ldots, h_{r}$ with pole data $\left(z_{2}, n_{2}\right), \ldots,\left(z_{r}, n_{r}\right)$ respectively such that

$$
g_{2} \cdots g_{r}=h_{2} *\left(h_{3} *\left(\cdots * h_{r}\right) \cdots\right) .
$$

Equation (6.2) implies that $\psi=f_{1} *\left(g_{2} \cdots g_{r}\right)$, which is equal to

$$
f_{1} *\left(h_{2} *\left(\cdots * h_{r}\right) \cdots\right) \text {. }
$$

q.e.d.

\section{Analytic BT and Holomorphic vector bundles}

In this section, we generalize some of Uhlenbeck's results on unitons to Ward solitons. In particular, we

1) derive the analytic Bäcklund transformation $\mathrm{BT}_{z, \psi}$ (given in the introduction) for the Ward equation,

2) associate to each Ward soliton and complex number $z \in \mathbb{C} \backslash \mathbb{R}$ a holomorphic structure on the trivial $\mathbb{C}^{n}$-bundle over $S^{2}$, 
3) prove that to find solutions $\pi$ of $\mathrm{BT}_{z, \psi}$ is equivalent to finding a one parameter family of holomorphic subbundles of the $\mathbb{C}^{n}$-bundle with respect to the holomorphic structure given in (2) that satisfy certain first order PDE system.

Suppose $\psi(\lambda)(x, y, t)=\psi(x, y, t, \lambda)$ is an extended solution of the Ward equation with $A=\left(\lambda \psi_{x}-\psi_{u}\right) \psi^{-1}$, and $B=\left(\lambda \psi_{v}-\psi_{x}\right) \psi^{-1}$. Motivated by the construction of Bäcklund transformations for soliton equations, we seek a new extended solution of the form $\psi_{1}(\lambda)=g_{z, \pi}(\lambda) \psi(\lambda)$ for some smooth map $\pi$ from $\mathbb{R}^{2,1}$ to the space of rank $k$ Hermitian projections of $\mathbb{C}^{n}$. The condition $\psi_{1}$ satisfies (1.4) implies that

$$
\left\{\begin{array}{l}
\left(\lambda g_{x}-g_{u}\right) g^{-1}+g A g^{-1}=\tilde{A} \\
\left(\lambda g_{v}-g_{x}\right) g^{-1}+g B g^{-1}=\tilde{B}
\end{array}\right.
$$

for some $\tilde{A}$ and $\tilde{B}$ independent of $\lambda$, where $g=g_{z, \pi}=\mathrm{I}+\frac{z-\bar{z}}{\lambda-z} \pi^{\perp}$. The reality condition implies that $g_{z, \pi}(\lambda)^{-1}=\mathrm{I}+\frac{\bar{z}-z}{\lambda-\bar{z}} \pi^{\perp}$. Since the right hand side of (7.1) is holomorphic in $\lambda \in \mathbb{C}$, the residue of the left hand side at $\lambda=z$ must be zero, which gives

$$
\left\{\begin{array}{l}
\left(z \pi_{x}^{\perp}-\pi_{u}^{\perp}\right) \pi+\pi^{\perp} A \pi=0, \\
\left(z \pi_{v}^{\perp}-\pi_{x}^{\perp}\right) \pi+\pi^{\perp} B \pi=0 .
\end{array}\right.
$$

But $\pi^{\perp} \pi=0$ implies that

$$
-\pi^{\perp}\left(z \pi_{x}-\pi_{u}\right)=\left(z \pi_{x}^{\perp}-\pi_{u}^{\perp}\right) \pi .
$$

So we get the following system of first order partial differential equations for $\pi$ :

$$
\left\{\begin{array}{l}
\pi^{\perp}\left(z \pi_{x}-\pi_{u}-A \pi\right)=0 \\
\pi^{\perp}\left(z \pi_{v}-\pi_{x}-B \pi\right)=0 .
\end{array}\right.
$$

The residue at $\lambda=\bar{z}$ is zero gives the same system (7.2), which is the analytic Bäcklund transformation (BT) for the Ward equation. So we have proved the following:

Proposition 7.1. Let $\psi$ be an extended solution of the Ward equation, $A=\left(\lambda \psi_{x}-\psi_{u}\right) \psi^{-1}$, and $B=\left(\lambda \psi_{v}-\psi_{x}\right) \psi^{-1}$. Given a smooth map $\pi$ from $\mathbb{R}^{2,1}$ to the space of Hermitian projections, $g_{z, \pi} \psi$ is an extended solution of the Ward equation if and only if $\pi$ satisfies (7.2).

Ioannidou and Zakrzewski proved the above proposition for $z=i$ in [8]. However, no general solutions of the system (7.2) were given.

As a consequence of Theorem 3.1 and Proposition 7.1 we have

Proposition 7.2. Suppose $\psi(x, u, v, \lambda)$ is an extended Ward map and is holomorphic and non-degenerate at $\lambda=z$, and $f_{1}, \ldots, f_{k}$ are meromorphic maps from $\mathbb{C}$ to $\mathbb{C}^{n}$ that are linearly independent except at finitely many points of $\mathbb{C}$. Let $w=x+z u+z^{-1} v, \tilde{f}_{i}=\psi(\cdots, z)\left(f_{i}\right)$, and 
$\tilde{\pi}$ be the Hermitian projection of $\mathbb{C}^{n}$ onto the span of $\tilde{f}_{1}, \ldots, \tilde{f}_{k}$. Then $\tilde{\pi}$ is a solution of $\mathrm{BT}_{z, \psi}$, or equivalently, $g_{z, \tilde{\pi}} \psi$ is again an extended solution.

Thus if $\psi$ is holomorphic and non-degenerate at $\lambda=z$, then the above proposition gives an algebraic method to construct solutions of $\mathrm{BT}_{z, \psi}$.

When $z=i, \pi$ is independent of $t$, and $\psi$ is an extended $k$-uniton, (7.2) is the singular BT used by Uhlenbeck in [10] to add one more uniton to the given $k$-uniton. She also proved that a solution of (7.2) for a uniton can be interpreted as a holomorphic subbundle that satisfies an algebraic constraint. In this section, we show that a solution $\pi$ of the analytic BT (7.2) for Ward map can also be interpreted in terms of holomorphic subbundle, but it now must satisfy a first order PDE constraint. We explain this next.

If we make a suitable linear change of coordinates of $\mathbb{R}^{2,1}$, then the operator $z \partial_{x}-\partial_{u}$ becomes a $\bar{\partial}$ operator. To see this, let $z=\alpha+i \beta$. Then

$$
w=x+z u+z^{-1} v=\left(x+\alpha u+\frac{\alpha v}{\alpha^{2}+\beta^{2}}\right)+i\left(\beta u-\frac{\beta v}{\alpha^{2}+\beta^{2}}\right) .
$$

Make a coordinate change:

$$
\left\{\begin{array}{l}
p=x+\alpha u+\frac{\alpha v}{\alpha^{2}+\beta^{2}} \\
q=\beta u-\frac{\beta v}{\alpha^{2}+\beta^{2}} \\
r=v
\end{array}\right.
$$

Then

$$
\left\{\begin{array}{l}
\partial_{x}=\partial_{p}, \\
\partial_{u}=\alpha \partial_{p}+\beta \partial_{q}, \\
\partial_{v}=\frac{\alpha}{\alpha^{2}+\beta^{2}} \partial_{p}-\frac{\beta}{\alpha^{2}+\beta^{2}} \partial_{q}+\partial_{r} .
\end{array}\right.
$$

A direct computation gives

$$
\begin{aligned}
z \partial_{x}-\partial_{u} & =(\alpha+i \beta) \partial_{p}-\left(\alpha \partial_{p}+\beta \partial_{q}\right)=i \beta \partial_{p}-\beta \partial_{q} \\
& =i \beta\left(\partial_{p}+i \partial_{q}\right)=2 i \beta \partial_{\bar{w}}, \\
z \partial_{v}-\partial_{x} & =(\alpha+i \beta)\left(\frac{\alpha}{\alpha^{2}+\beta^{2}} \partial_{p}-\frac{\beta}{\alpha^{2}+\beta^{2}} \partial_{q}+\partial_{r}\right)-\partial_{p} \\
& =\frac{2 i \beta}{\alpha-i \beta}\left(\partial_{\bar{w}}-\frac{\alpha^{2}+\beta^{2}}{2 i \beta} \partial_{r}\right) .
\end{aligned}
$$

Use $z=\alpha+i \beta$ and substitute the above formulas into the analytic BT (7.2) to get

$$
\left\{\begin{array}{l}
\pi^{\perp}\left(\partial_{\bar{w}}-\frac{A}{z-\bar{z}}\right) \pi=0, \\
\pi^{\perp}\left(\partial_{\bar{w}}+\frac{|z|^{2}}{z-\bar{z}} \partial_{r}-\frac{\bar{z}}{z-\bar{z}} B\right) \pi=0 .
\end{array}\right.
$$


Let

Then

$$
\left\{\begin{array}{l}
\mathcal{L}=\partial_{\bar{w}}-\frac{A}{z-\bar{z}}, \\
\mathcal{M}=\partial_{\bar{w}}+\frac{|z|^{2}}{z-\bar{z}} \partial_{r}-\frac{\bar{z} B}{z-\bar{z}} .
\end{array}\right.
$$

$$
\mathcal{M}-\mathcal{L}=\frac{|z|^{2}}{z-\bar{z}} \partial_{r}+\frac{1}{z-\bar{z}}(A-\bar{z} B) .
$$

So system (7.4) is equivalent to

$$
\left\{\begin{array}{l}
\pi^{\perp}\left(\partial_{\bar{w}}-\frac{A}{z-\bar{z}}\right) \pi=0 \\
\pi^{\perp}\left(\partial_{r}+\frac{1}{|z|^{2}}(A-\bar{z} B)\right) \pi=0
\end{array}\right.
$$

Thus we have shown

Proposition 7.3. Let $\psi, A, B$ be as in Proposition 7.1. Then $g_{z, \pi} \psi$ is an extended solution of the Ward equation if and only if $\pi$ satisfies (7.5).

The first equation of (7.5) has an interpretation in terms of holomorphic subbundle. To explain this, we first review some notation of holomorphic vector bundles over $S^{2}$ (cf. [10], [15]). A map $\eta$ defined and is smooth on $S^{2}$ except on a finite subset $D$ is said to be of pole type if at each $p_{0} \in D$ there exists a local complex coordinate $(\mathcal{O}, w)$ of $S^{2}$ at $p_{0}$ with $w\left(p_{0}\right)=0$ such that the map $\eta(w)=w^{-m} \eta_{0}(w)$ for all $w \in \mathcal{O}$, where $m$ is some positive integer and $\eta_{0}$ is smooth in a neighborhood of 0 . Point $p_{0}$ is called a pole of $\eta$.

Given a smooth map $f: S^{2} \rightarrow U(n)$ and a constant $c$, let $A=c f_{\bar{w}} f^{-1}$; then $\partial_{\bar{w}}-A$ gives a holomorphic structure on the trivial bundle $\underline{\mathbb{C}^{n}}=$ $S^{2} \times \mathbb{C}^{n}$ over $S^{2}$. A local section $\xi$ of $\underline{\mathbb{C}^{n}}$ is holomorphic in the complex structure $\partial_{\bar{w}}-A$ if

$$
\partial_{\bar{w}} \xi-A \xi=0 .
$$

A meromorphic section of a holomorphic vector bundle is a section of pole type and is holomorphic away from the poles. It is known that the space of meromorphic sections of a rank $k$ holomorphic vector bundle $E$ over $S^{2}$ is of dimension $k$ over the field $\mathcal{R}\left(S^{2}\right)$ of meromorphic functions on $S^{2}$. In other words, there exist $k$ meromorphic sections $\eta_{1}, \ldots, \eta_{k}$ such that if $\eta$ is a meromorphic section of $E$ then there exist $f_{1}, \ldots, f_{k}$ in $\mathcal{R}\left(S^{2}\right)$ so that $\eta=\sum_{j=1}^{k} f_{j} \eta_{j}$. We call such $\left\{\eta_{1}, \ldots, \eta_{k}\right\}$ a meromorphic frame of $E$.

The following is known (cf. $[\mathbf{1 0}, \mathbf{1 5}])$ :

Proposition 7.4. Given a smooth map $\pi: S^{2} \rightarrow \operatorname{Gr}\left(k, \mathbb{C}^{n}\right)$, let $\Pi$ denote the subbundle of $\underline{\mathbb{C}^{n}}$ whose fiber over $p \in S^{2}$ is $\operatorname{Im}(\pi(p))$. Then the following two statements are equivalent:

1) $\pi^{\perp}\left(\partial_{\bar{w}}-A\right) \pi=0$,

2) $\Pi$ is a rank $k$ holomorphic subbundle of $\underline{\mathbb{C}^{n}}$ with respect to $\partial_{\bar{w}}-A$. 
Moreover, if (1) or (2) holds, then there exist maps $\xi_{1}, \ldots, \xi_{k}: S^{2} \rightarrow \mathbb{C}^{n}$ of pole type so that

(a) $\xi_{1}(p), \ldots, \xi_{k}(p)$ span $\operatorname{Im}(\pi(p))$ for all $p \in S^{2}$ except at finitely many points,

(b) each $\xi_{j}$ is a solution of $\partial_{\bar{w}} \eta-A \eta=0$,

(c) every meromorphic section $\eta$ of $\Pi$ is of the form $\sum_{j=1}^{k} f_{j} \xi_{j}$ for some $f_{1}, \ldots, f_{k} \in \mathcal{R}\left(S^{2}\right)$.

As a consequence of the discussion above, we have

Corollary 7.5. Let $\psi$ be an extended Ward soliton with $A=\left(\lambda \psi_{x}-\right.$ $\left.\psi_{u}\right) \psi^{-1}$ and $B=\left(\lambda \psi_{v}-\psi_{x}\right) \psi^{-1}$, and $(p, q, r)$ the coordinate system on $\mathbb{R}^{2,1}$ defined by (7.3). Let $\pi: \mathbb{R}^{2,1} \rightarrow \operatorname{Gr}\left(k, \mathbb{C}^{n}\right)$ be a smooth map that extends to $S^{2} \times \mathbb{R}$. Then the following statements are equivalent:

1) $\pi$ is a solution of $(7.2)$.

2) For each fixed $r$, the subbundle $\Pi(r)$ associated to $\pi(\cdot, \cdot, r)$ is a holomorphic subbundle of $\underline{\mathbb{C}^{n}}$ in the complex structure $\partial_{\bar{w}}-\frac{A}{z-\bar{z}}$ and satisfies $\pi^{\perp}\left(\partial_{r}+\frac{1}{|z|^{2}}(A-\bar{z} B)\right) \pi=0$.

3) There exist maps $\xi_{1}, \ldots, \xi_{k}: S^{2} \times \mathbb{R} \rightarrow \mathbb{C}^{n}$ satisfying the following conditions:

a) $\left\{\xi_{1}(\cdot, r), \ldots, \xi_{k}(\cdot, r)\right\}$ is a meromorphic frame of $\Pi(r)$,

b) $\partial_{r} \xi_{j}+\frac{1}{|z|^{2}}(A-\bar{z} B) \xi_{j}$ is a section of $\Pi$ of pole type for all $1 \leq j \leq k$.

\section{Construction of all Ward solitons}

The goal of this section is to show that all Ward solitons can be constructed by the methods given in sections 3,5 and 6 (using algebraic BTs, limiting method, and generalized algebraic BTs). By Proposition 6.7 , it suffices to show that for any given $z \in \mathbb{C}_{+}$and $k \in \mathbb{N}$, we can construct all extended Ward solitons of pole data $(z, k)$.

First we prove

Theorem 8.1. If $\phi_{k}$ is an extended solution of the Ward equation and $\phi_{k}=g_{z, \pi_{k}} \cdots g_{z, \pi_{1}}$ is the minimal factorization, then the tails of $\phi_{k}$, $\phi_{l}=g_{z, \pi_{l}} \cdots g_{z, \pi_{1}}, l=1, \ldots, k-1$, are also extended solutions.

Proof. We prove the theorem by induction on $l$. Since $\phi_{k}$ is an extended solution,

$$
\left\{\begin{array}{l}
A_{k}=\left(\lambda \partial_{x} \phi_{k}-\partial_{u} \phi_{k}\right) \phi_{k}^{-1}, \\
B_{k}=\left(\lambda \partial_{v} \phi_{k}-\partial_{x} \phi_{k}\right) \phi_{k}^{-1},
\end{array}\right.
$$

are independent of $\lambda$. We want to prove $g_{z, \pi_{1}}$ is an extended solution. Compute the Laurent series expansion of $\phi_{k}$ at $\lambda=z$ to get

$$
\phi_{k}=\mathrm{I}+\frac{z-\bar{z}}{\lambda-z} P_{1}+\cdots+\left(\frac{z-\bar{z}}{\lambda-z}\right)^{k} P_{k},
$$


where

$$
P_{j}=\sum_{k \geq i_{1}>\cdots>i_{j} \geq 1} \pi_{i_{1}}^{\perp} \cdots \pi_{i_{j}}^{\perp} .
$$

Compute the Laurent series expansion of both sides of

$$
\lambda \partial_{x} \phi_{k}-\partial_{u} \phi_{k}=A_{k} \phi_{k}
$$

at $\lambda=z$, and compare the coefficients of $(\lambda-z)^{-k}$ to see

$$
\left(z \partial_{x}-\partial_{u}\right) P_{k}=A_{k} P_{k}
$$

where $P_{k}=\pi_{k}^{\perp} \cdots \pi_{1}^{\perp}$. Multiply $\pi_{1}$ from right to both sides of (8.4) to see

$$
\pi_{k}^{\perp} \cdots \pi_{2}^{\perp}\left(\left(z \partial_{x}-\partial_{u}\right) \pi_{1}^{\perp}\right) \pi_{1}=0 .
$$

But $\pi_{1}^{\perp} \pi_{1}=0$ implies

$$
L_{z}\left(\pi_{1}^{\perp}\right) \pi_{1}+\pi_{1}^{\perp} L_{z}\left(\pi_{1}\right)=0,
$$

where $L_{z}=z \partial_{x}-\partial_{u}$. So we have

$$
\pi_{k}^{\perp} \cdots \pi_{1}^{\perp} L_{z}\left(\pi_{1}\right)=0 .
$$

By assumption, $\phi_{k}=g_{z, \pi_{k}} \cdots g_{z, \pi_{1}}$ is a minimal factorization, i.e., $\operatorname{Im} \pi_{j} \cap$ $\operatorname{Im} \pi_{j-1}^{\perp}=0$. So by Lemma 4.8,

$$
\operatorname{Ker}\left(\pi_{k}^{\perp} \cdots \pi_{1}^{\perp}\right)=\operatorname{Im} \pi_{1} .
$$

Hence equation (8.5) implies that

$$
\operatorname{Im}\left(L_{z}\left(\pi_{1}\right)\right) \subset \operatorname{Im} \pi_{1} .
$$

So

$$
\pi_{1}^{\perp}\left(z \partial_{x} \pi_{1}-\partial_{u} \pi_{1}\right)=0 .
$$

Use the second equation of (8.1) and similar argument to prove that

$$
\pi_{1}^{\perp}\left(z \partial_{v} \pi_{1}-\partial_{x} \pi_{1}\right)=0 .
$$

The above two equalities imply that $\phi_{1}=g_{z, \pi_{1}}$ is an extended solution of Ward equation.

Assume that $\phi_{l}$ is an extended solution, and let

$$
A_{l}=\left(\lambda \partial_{x} \phi_{l}-\partial_{u} \phi_{l}\right) \phi_{l}^{-1}, \quad B_{l}=\left(\lambda \partial_{v} \phi_{l}-\partial_{x} \phi_{l}\right) \phi_{l}^{-1} .
$$

We want to show that $\phi_{l+1}$ is an extended solution too. Write $\phi_{k}=$ $\psi_{k-l} \phi_{l}$, where $\psi_{k-l}=g_{z, \pi_{k}} \cdots g_{z, \pi_{l+1}}$. Substitute $\phi_{k}=\psi_{k-l} \phi_{l}$ to (8.3) to get

$$
\left(\lambda \partial_{x} \psi_{k-l}-\partial_{u} \psi_{k-l}\right) \phi_{l}+\psi_{k-l}\left(\lambda \partial_{x} \phi_{l}-\partial_{u} \phi_{l}\right)=A_{k} \psi_{k-l} \phi_{l} .
$$

Multiply $\phi_{l}^{-1}$ from the right to both sides and use $\lambda \partial_{x} \phi_{l}-\partial_{u} \phi_{l}=A_{l} \phi_{l}$ to get

$$
\left(\lambda \partial_{x} \psi_{k-l}-\partial_{u} \psi_{k-l}\right)+\psi_{k-l} A_{l}=A_{k} \psi_{k-l}
$$


Compute the Laurent series expansion of both sides at $\lambda=z$, and compare the coefficients of $(\lambda-z)^{-(k-l)}$ to get

$$
\left(z \partial_{x}-\partial_{u}\right)\left(\pi_{k}^{\perp} \cdots \pi_{l+1}^{\perp}\right)+\pi_{k}^{\perp} \cdots \pi_{l+1}^{\perp} A_{l}=A_{k} \pi_{k}^{\perp} \cdots \pi_{l+1}^{\perp} .
$$

Multiply $\pi_{l+1}$ from right to both sides to get

$$
\pi_{k}^{\perp} \cdots \pi_{l+1}^{\perp}\left(z \partial_{x} \pi_{l+1}-\partial_{u} \pi_{l+1}-A_{l} \pi_{l+1}\right)=0 .
$$

By Lemma 4.8, we have

$$
\operatorname{Im}\left(z \partial_{x} \pi_{l+1}-\partial_{u} \pi_{l+1}-A_{l} \pi_{l+1}\right) \subset \operatorname{Ker}\left(\pi_{k}^{\perp} \cdots \pi_{l+1}^{\perp}\right)=\operatorname{Im} \pi_{l+1} .
$$

Thus we have

$$
\pi_{l+1}^{\perp}\left(z \partial_{x} \pi_{l+1}-\partial_{u} \pi_{l+1}-A_{l} \pi_{l+1}\right)=0 .
$$

Likewise, from $B_{k}$ we can obtain

$$
\pi_{l+1}^{\perp}\left(z \partial_{v} \pi_{l+1}-\partial_{x} \pi_{l+1}-B_{l} \pi_{l+1}\right)=0 .
$$

The above two equalities imply that $\pi_{l+1}$ is a solution of the analytic BT (7.2) with $(A, B)=\left(A_{l}, B_{l}\right)$, hence $\phi_{l+1}=g_{z, \pi_{l+1}} \phi_{l}$ is an extended solution. Thus we complete the proof by induction.

Theorem 8.1 tells us that any extended solution of pole data $(z, k)$ is obtained by solving the analytic BT (7.2) of an extended 1-soliton, then of an extended 2-soliton, ... etc.

The following two lemmas prove that to solve the analytic BT (7.2), which is a system of non-linear equations, it suffices to solve a certain first order linear system.

Lemma 8.2. Let $\psi$ be an extended solution of the Ward equation, and $A=\left(\lambda \psi_{x}-\psi_{u}\right) \psi^{-1}$, and $B=\left(\lambda \psi_{v}-\psi_{x}\right) \psi^{-1}$. If $\pi$ is a local solution of $(7.2)$, then there exists a local $\mathcal{M}_{n \times r}^{0}(\mathbb{C})$-valued smooth map $V$ so that columns of $V$ span $\operatorname{Im} \pi$ and $V$ satisfies

$$
\left\{\begin{array}{l}
z V_{x}-V_{u}-A V=0 \\
z V_{v}-V_{x}-B V=0 .
\end{array}\right.
$$

Conversely, if $V$ is a solution of (8.6), then the Hermitian projection $\pi$ of $\mathbb{C}^{n}$ onto $V$ is a solution of (7.2). In fact, $\pi=V\left(V^{*} V\right)^{-1} V^{*}$.

Proof. Choose a local $\mathcal{M}_{n \times r}^{0}(\mathbb{C})$-valued smooth map $V$ such that columns of $V$ span $\operatorname{Im} \pi$. So $\pi=V\left(V^{*} V\right)^{-1} V^{*}$. Substitute this into (7.2) to see

$$
\left\{\begin{array}{l}
\pi^{\perp}\left(z V_{x}-V_{u}-A V\right)\left(V^{*} V\right)^{-1} V^{*}=0 \\
\pi^{\perp}\left(z V_{v}-V_{x}-B V\right)\left(V^{*} V\right)^{-1} V^{*}=0
\end{array}\right.
$$

Multiply $V$ from the right to see

$$
\left\{\begin{array}{l}
\pi^{\perp}\left(z V_{x}-V_{u}-A V\right)=0 \\
\pi^{\perp}\left(z V_{v}-V_{x}-B V\right)=0 .
\end{array}\right.
$$


This implies that $\operatorname{Im}\left(z V_{x}-V_{u}-A V\right)$ and $\operatorname{Im}\left(z V_{v}-V_{x}-B V\right)$ lie in $\operatorname{Im} \pi$. So

$$
\left\{\begin{array}{l}
z V_{x}-V_{u}-A V=V h, \\
z V_{v}-V_{x}-B V=V k,
\end{array}\right.
$$

for some $r \times r$ matrix-valued maps $h, k$.

Claim that there exists a smooth $G L(r, \mathbb{C})$-valued map $\phi$ so that $\tilde{V}=V \phi$ satisfies (8.6). To see this, let

$$
L_{1}=z \partial_{x}-\partial_{u}, \quad L_{2}=z \partial_{v}-\partial_{x} .
$$

Since $L_{1}, L_{2}$ are constant coefficient linear operators, they commute. A direct computation shows that $V \phi$ satisfies (8.6) if and only if $\phi$ satisfies

$$
\left\{\begin{array}{l}
L_{1} \phi=-h \phi \\
L_{2} \phi=-k \phi
\end{array}\right.
$$

Equation (8.9) is solvable if and only if $h, k$ satisfy

$$
-L_{2}(h)+h k=-L_{1}(k)+k h .
$$

This condition for $h, k$ comes from equating $L_{1} L_{2} \phi=L_{2} L_{1} \phi$.

Write (8.8) in terms of $L_{1}, L_{2}$ to get

$$
\left\{\begin{array}{l}
L_{1}(V)=A V+V h, \\
L_{2}(V)=B V+V k .
\end{array}\right.
$$

Since $L_{1}, L_{2}$ commute, we have

$$
\begin{aligned}
L_{2} L_{1}(V) & =L_{2}(A) V+A L_{2}(V)+L_{2}(V) h+V L_{2}(h) \\
& =\left(L_{2}(A)+A B\right) V+A V k+(B V+V k) h+V L_{2}(h) \\
=L_{1} L_{2}(V) & =L_{1}(B) V+B L_{1}(V)+L_{1}(V) k+V L_{1}(k) \\
& =\left(L_{1}(B)+B A\right) V+B V h+(A V+V h) k+V L_{1}(k) .
\end{aligned}
$$

Hence

$$
\left(L_{2}(A)-L_{1}(B)+[A, B]\right) V=V\left(L_{1}(k)-L_{2}(h)+[h, k]\right) .
$$

Since $\psi$ is an extended Ward map, $A, B$ satisfies (1.4), which implies that

$$
L_{2}(A)-L_{1}(B)+[A, B]=0 .
$$

By (8.11) and (8.12), $h, k$ satisfy (8.10). Thus we can find local smooth solution $\phi$ for (8.9) and $\tilde{V}=V \phi$ solves (8.6).

The converse is clearly true.

q.e.d.

It is easy to see that solutions of (8.6) are unique up to $V \rightarrow V H$, where $H$ is a map from $\mathbb{R}^{2,1}$ to $G L(r, \mathbb{C})$ satisfying

$$
\left\{\begin{array}{l}
z H_{x}-H_{u}=0, \\
z H_{v}-H_{x}=0
\end{array}\right.
$$


i.e., $H$ depends on $w=x+z u+z^{-1} v$ only.

If we can construct local fundamental solutions to the linear system (8.6), then we can obtain all local extended solutions of the form $g_{z, \pi} \psi$. But in order to construct global Ward maps satisfying the boundary condition (1.8), we need to construct fundamental solutions that are of pole type on each $w$-plane.

If $\psi$ is an extended Ward soliton, holomorphic and non-degenerate at $\lambda=z$, then $\psi(z)$ itself is a fundamental solution to (8.6). So we have:

Proposition 8.3. Let $\psi$ be an extended Ward soliton, and $A=$ $\left(\lambda \psi_{x}-\psi_{u}\right) \psi^{-1}$ and $B=\left(\lambda \psi_{v}-\psi_{x}\right) \psi^{-1}$. If $\psi(x, u, v, \lambda)$ is holomorphic and non-degenerate at $\lambda=z$, then $\psi(\cdots, z)$ is a fundamental solution of (8.6). Consequently, the columns of $\psi(\cdots, z)$ form a holomorphic frame of the trivial $\mathbb{C}^{n}$-bundle over $S^{2}$ with respect to the complex structure $\partial_{\bar{w}}-\frac{A}{z-\bar{z}}$ on each $w$-plane.

Proof. Since $\psi$ is holomorphic and non-degenerate at $\lambda=z,(1.4)$ implies that

$$
\left\{\begin{array}{l}
z \psi_{x}(z)-\psi_{u}(z)-A \psi(z)=0 \\
z \psi_{v}(z)-\psi_{u}(z)-B \psi(z)=0
\end{array}\right.
$$

Hence the columns of $\psi(\cdots, z)$ are smooth solutions of (8.6). So on each $w$-plane, columns of $\psi(z)$ form a holomorphic frame of the trivial $\mathbb{C}^{n}$-bundle over $S^{2}$ with respect to the complex structure $\partial_{\bar{w}}-\frac{A}{z-\bar{z}}$.

q.e.d.

The above proposition implies that Theorem 3.1 gives all extended solutions of the form $g_{z, \pi} \psi$ when $\psi$ is holomorphic and non-degenerate at $\lambda=z$.

If $\psi$ is an extended Ward soliton and has a pole at $\lambda=z$, then we will show below that the limiting method used in section 5 gives fundamental solutions of (8.6) that are of pole type on each $w$-plane.

We need two lemmas first, and their proofs are straight forward.

Lemma 8.4. Let $b_{\epsilon}, \eta_{\epsilon}$ and $\psi_{\epsilon}$ be maps from $\mathbb{R}^{2,1} \times \Omega$ to $g l(n, \mathbb{C})$, where $\Omega$ is an open subset of 0 in $\mathbb{C}$. Suppose $b_{\epsilon}=\sum_{j=0}^{\infty} c_{j} \epsilon^{j}, \psi_{\epsilon}=$ $\sum_{j=0}^{k} P_{j} \epsilon^{-j}$, and $\eta_{\epsilon}=\psi_{\epsilon}\left(b_{\epsilon}\right)$. If $\psi_{\epsilon}\left(b_{\epsilon}\right)$ is smooth at $\epsilon=0$, i.e., $\sum_{j-i=m} P_{j} c_{i}=0$ for all $m>0$, then

1) $\eta:=\lim _{\epsilon \rightarrow 0} \eta_{\epsilon}=\sum_{j=0}^{k} P_{j} c_{j}$,

2) $\left\{\begin{array}{l}z \eta_{x}-\eta_{u}=\lim _{\epsilon \rightarrow 0}(z+\epsilon)\left(\eta_{\epsilon}\right)_{x}-\left(\eta_{\epsilon}\right)_{u} \\ z \eta_{v}-\eta_{x}=\lim _{\epsilon \rightarrow 0}(z+\epsilon)\left(\eta_{\epsilon}\right)_{v}-\left(\eta_{\epsilon}\right)_{x} .\end{array}\right.$

Lemma 8.5. Let $w_{\epsilon}=x+(z+\epsilon) u+(z+\epsilon)^{-1} v, b_{i \epsilon}=\sum_{j=0}^{k_{i}} \epsilon^{j} a_{i j}\left(w_{\epsilon}\right)$ for $i=1,2, \phi$ an extended Ward map, and $f_{1}, f_{2}$ meromorphic functions 
from $\mathbb{C}$ to $\mathbb{C}$. If $\lim _{\epsilon \rightarrow 0} \phi(z+\epsilon)\left(b_{i \epsilon}\right)=\eta_{i}$ exists for $i=1,2$, then

$$
\lim _{\epsilon \rightarrow 0} \phi(z+\epsilon)\left(f_{1}\left(w_{\epsilon}\right) b_{1 \epsilon}+f_{2}\left(w_{\epsilon}\right) b_{2 \epsilon}\right)=f_{1} \eta_{1}+f_{2} \eta_{2} .
$$

Proof. The lemma follows from

$$
\begin{aligned}
& \phi(z+\epsilon)\left(f_{1}\left(w_{\epsilon}\right) b_{1 \epsilon}+f_{2}\left(w_{\epsilon}\right) b_{2 \epsilon}\right) \\
& =f_{1}\left(w_{\epsilon}\right) \phi(z+\epsilon)\left(b_{1 \epsilon}\right)+f_{2}\left(w_{\epsilon}\right) \phi(z+\epsilon)\left(b_{2 \epsilon}\right) \\
& \quad \rightarrow f_{1}(w) \eta_{1}+f_{2}(w) \eta_{2} \quad \text { as } \quad \epsilon \rightarrow 0 .
\end{aligned}
$$

q.e.d.

Theorem 8.6. Let $\phi_{k}$ be an extended Ward soliton with pole data $(z, k)$, and

$$
A_{k}=\left(\lambda\left(\phi_{k}\right)_{x}-\left(\phi_{k}\right)_{u}\right) \phi_{k}^{-1}, \quad B_{k}=\left(\lambda\left(\phi_{k}\right)_{v}-\left(\phi_{k}\right)_{x}\right) \phi_{k}^{-1} .
$$

Then

1) $\phi_{k}$ can be constructed using algebraic BT and the limiting method given in section 5 ,

2) we can use algebraic $B T$ and the limiting method to construct a fundamental solution $\eta$ of (8.6) with $A=A_{k}, B=B_{k}$, and the entries of $\eta$ are rational functions in $x, y$ and $t$.

Proof. We prove the theorem by induction on $k$. For $k=1,(1)$ is obvious. For (2), we first choose $\mathbb{C}^{n}$-valued rational maps $b_{n_{1}+1}, \ldots, b_{n}$ such that $\pi_{1}^{\perp}\left(b_{n_{1}+1}\right), \ldots, \pi_{1}^{\perp}\left(b_{n}\right)$ span $\operatorname{Im} \pi_{1}^{\perp}$, where $n_{1}=\operatorname{rk}\left(\pi_{1}\right)$. Let

$$
u_{j, \epsilon}=g_{z, \pi_{1}}(z+\epsilon)\left(\epsilon b_{j}\left(w_{\epsilon}\right)\right) .
$$

When $|\epsilon|>0$ is small, $g_{z, \pi_{1}}$ is holomorphic and non-degenerate at $\lambda=$ $z+\epsilon$. Hence by Proposition 8.3, we have

$$
\left\{\begin{array}{l}
(z+\epsilon)\left(u_{j, \epsilon}\right)_{x}-\left(u_{j, \epsilon}\right)_{u}-A_{1} u_{j, \epsilon}=0, \\
(z+\epsilon)\left(u_{j, \epsilon}\right)_{v}-\left(u_{j, \epsilon}\right)_{v}-B_{1} u_{j, \epsilon}=0 .
\end{array}\right.
$$

A direct computation implies that

$$
u_{j}=\lim _{\epsilon \rightarrow 0} u_{j, \epsilon}=(z-\bar{z}) \pi_{1}^{\perp}\left(b_{j}(w)\right) .
$$

As $\epsilon \rightarrow 0$, Lemma 8.4 implies that $u_{j}$ satisfies

$$
\left\{\begin{array}{l}
z\left(u_{j}\right)_{x}-\left(u_{j}\right)_{u}-A_{1} u_{j}=0, \\
z\left(u_{j}\right)_{v}-\left(u_{j}\right)_{x}-B_{1} u_{j}=0,
\end{array}\right.
$$

for $n_{1}+1 \leq j \leq n$. So $u_{n_{1}+1}, \ldots, u_{n}$ are rational maps in $x, y, t$ and are linearly independent solutions of (8.6) with $A=A_{1}$ and $B=B_{1}$.

We claim that by choosing a sequence of rational functions carefully, we can construct the rest linearly independent solutions of (8.6). Let 
$a_{1}, \ldots, a_{n_{1}}$ be $\mathbb{C}^{n}$-valued rational maps that span $\operatorname{Im} \pi_{1}$ except at finitely many points. Use formulas in Section 5 to get

$$
\begin{aligned}
& g_{z, \pi_{1}}(z+\epsilon)\left(a_{j}\left(w_{\epsilon}\right)\right) \\
& =\left(\mathrm{I}+\frac{z-\bar{z}}{\epsilon} \pi_{1}^{\perp}\right)\left(a_{j}(w)+\left(u-z^{-2} v\right) a_{j}^{\prime}(w) \epsilon+O\left(\epsilon^{2}\right)\right) .
\end{aligned}
$$

So

$$
\eta_{j}:=\lim _{\epsilon \rightarrow 0} g_{z, \pi_{1}}(z+\epsilon)\left(a_{j}\left(w_{\epsilon}\right)\right)=a_{j}(w)+(z-\bar{z})\left(u-z^{-2} v\right) \pi_{1}^{\perp}\left(a_{j}^{\prime}(w)\right)
$$

is rational in $x, y$ and $t$. By Proposition 8.3 and Lemma $8.4, \eta_{j}$ is a solution of (8.6) with $A=A_{1}$ and $B=B_{1}$. Because $u_{\alpha}$ with $n_{1}+1 \leq \alpha \leq n$ $\operatorname{span} \operatorname{Im} \pi_{1}^{\perp}$ and $\pi_{1}\left(\eta_{1}\right), \ldots, \pi_{1}\left(\eta_{n_{1}}\right)$ span $\operatorname{Im} \pi_{1},\left\{\eta_{1}, \ldots, \eta_{n_{1}}, u_{n_{1}+1}, \ldots u_{n}\right\}$ form a fundamental solution of (8.6). We have proved that the entries of $\eta_{i}$ and $u_{j}$ are rational in $x, y$ and $t$. This proves the claim and the theorem for $k=1$.

Suppose the theorem is true for $k$. We want to prove that (1) holds for $k+1$. We may assume that

$$
\phi_{k+1}=g_{z, \pi_{k+1}} \cdots g_{z, \pi_{1}}
$$

is the minimal factorization. Let $n_{j}=\operatorname{rk}\left(\pi_{j}\right)$. By Theorem 8.1, $\phi_{k}=$ $g_{z, \pi_{k}} \cdots g_{z, \pi_{1}}$ is also an extended solution and a minimal factorization. By induction hypothesis, we can construct a fundamental solution $\eta=$ $\left(\eta_{1}, \ldots, \eta_{n}\right)$ rational in $x, y, t$ for $(8.6)$ with $A=A_{k}$ and $B=B_{k}$. Since $\phi_{k}$ and $g_{z, \pi_{k+1}} \phi_{k}$ are extended solutions, by Proposition $7.1, \pi_{k+1}$ is a solution of (7.2). By Proposition 7.4, there exist maps $\xi_{1}, \ldots, \xi_{n_{k+1}}$ of pole type that span $\operatorname{Im} \pi_{k+1}$ and satisfy (8.6) with $A=A_{k}$ and $B=$ $B_{k}$. But $\eta$ is a fundamental solution of (8.6) over the field $\mathcal{R}\left(S^{2}\right)$. So there exists a rational map $h=\left(h_{i j}\right)$ from $\mathbb{C}$ to $\mathcal{M}_{n \times n_{k+1}}^{0}(\mathbb{C})$ such that $\xi_{j}=\sum_{i=1}^{n} h_{i j} \eta_{i}$ for $1 \leq j \leq n_{k+1}$. By induction hypothesis, each $\eta_{i}$ is constructed by the limiting method. It follows from Lemma 8.5 that $\xi_{j}$ can be constructed by the limiting method. This proves (1) for $k+1$.

To prove (2), let $g=g_{z, \pi_{k+1}}$. Note that $\phi_{k+1}=g \phi_{k}$ implies

$$
L_{\lambda}(g) g^{-1}+g L_{\lambda}\left(\phi_{k}\right) \phi_{k}^{-1} g^{-1}=A_{k+1},
$$

where $L_{\lambda}(\xi)=\lambda \xi_{x}-\xi_{u}$. But $L_{\lambda}\left(\phi_{k}\right) \phi_{k}^{-1}=A_{k}$. So we have

$$
L_{\lambda}(g)+g A_{k}=A_{k+1} g .
$$

Equate the residue of the above equation at $\lambda=z$ to get

$$
L_{z}\left(\pi_{k+1}^{\perp}\right)+\pi_{k+1}^{\perp} A_{k}=A_{k+1} \pi_{k+1}^{\perp} .
$$

We have $L_{z}(\eta)=A_{k} \eta$. Set $W=\pi_{k+1}^{\perp} \eta$, where $\eta$ is a fundamental solution of (8.6) for $\phi_{k}$. We want to show that $W$ satisfies (8.6) with 
$A=A_{k+1}$ and $B=B_{k+1}$. To see this, we compute

$$
\begin{aligned}
L_{z} W-A_{k+1} W & =L_{z}\left(\pi_{k+1}^{\perp} \eta\right)-A_{k+1} \pi_{k+1}^{\perp} \eta \\
& =L_{z}\left(\pi_{k+1}^{\perp}\right) \eta+\pi_{k+1}^{\perp} L_{z}(\eta)-A_{k+1} \pi_{k+1}^{\perp} \eta \\
& =\left(L_{z}\left(\pi_{k+1}^{\perp}\right)+\pi_{k+1}^{\perp} A_{k}-A_{k+1} \pi_{k+1}^{\perp}\right) \eta,
\end{aligned}
$$

which is zero by (8.13). Similar argument implies that $z W_{v}-W_{x}-$ $B_{k+1} W=0$. This proves the claim. We may assume that the columns $\zeta_{1}, \ldots, \zeta_{n-n_{k+1}}$ of $W$ are linearly independent. So these columns are linearly independent solutions of (8.6) with $A=A_{k+1}$ and $B=B_{k+1}$. Since $\pi_{k+1}$ is constructed by limiting method, so are the $\zeta_{j}$ 's. It remains to construct $n_{k+1}$ other linearly independent solutions of (8.6). We have proved (1) for $k+1$. So there exist

$$
b_{j, \epsilon}=\sum_{i=0}^{k} \epsilon^{i} a_{j i}\left(w_{\epsilon}\right)
$$

with rational maps $a_{j i}$ from $\mathbb{C}$ to $\mathbb{C}^{n}$ so that

$$
\phi_{k}(z+\epsilon)\left(b_{j, \epsilon}\right)=\xi_{j}+\epsilon y_{j}+O\left(\epsilon^{2}\right),
$$

and $\xi_{1}, \ldots, \xi_{n_{k+1}} \operatorname{span} \operatorname{Im} \pi_{k+1}$. The limit of

$$
\phi_{k+1}(z+\epsilon)\left(b_{j, \epsilon}\right)=\left(\mathrm{I}+\frac{z-\bar{z}}{\epsilon} \pi_{k+1}^{\perp}\right)\left(\xi_{j}+\epsilon y_{j}+O\left(\epsilon^{2}\right)\right)
$$

as $\epsilon \rightarrow 0$ is

$$
\eta_{j}=\xi_{j}+(z-\bar{z}) \pi_{k+1}^{\perp}\left(y_{j}\right), \quad 1 \leq j \leq n_{k+1} .
$$

By Lemma 8.4, these $\eta_{j}$ 's are solutions of (8.6). So

$$
\eta_{1}, \ldots, \eta_{n_{k+1}}, \zeta_{1}, \ldots, \zeta_{n-n_{k+1}}
$$

form a fundamental solution of (8.6) with $A=A_{k+1}$ and $B=B_{k+1}$. The above arguments also prove that all entries of $\eta_{i}$ and $\zeta_{j}$ are rational in $x, y$ and $t$. This completes the proof of the Theorem.

q.e.d.

As a consequence of Proposition 6.7 and Theorem 8.6, we have

Corollary 8.7. All Ward solitons can be constructed by algebraic Bäcklund transformations, the limiting method and generalized Bäcklund transformations in Sections 3, 5 and 6. Moreover, the entries of Ward solitons are rational functions in $x, y$ and $t$.

\section{An explicit construction of unitons}

We have proved in the last section that all Ward solitons of pole type $(i, k)$ can be constructed by the limiting method of Section 5 . Since $U(n)$-unitons are stationary Ward solitons with pole type $(i, k)$ for some $k<n$, we can use our method to construct unitons. In this section, we write down the conditions on the sequence of rational maps 
so that the limiting Ward solitons are independent of $t$. We then give explicit formulas for unitons arising from finite sequence of $\mathbb{C}^{n}$-valued rational maps. We note that Wood [15] and Burstall-Guest [4] also gave algorithms to construct unitons. Our construction is somewhat different from theirs.

It is proved by Uhlenbeck that every $k$-uniton has a unique extended solution of the form $\phi_{k}=g_{i, \pi_{k}} \cdots g_{i, \pi_{1}}$ with the property that the span of

$$
\left\{\pi_{i} \pi_{i-1} \cdots \pi_{1}(w) \mid w \in S^{2}\right\}
$$

is $\mathbb{C}^{n}$ for each $1 \leq i \leq k$. Moreover, $\operatorname{rk} \pi_{1}>\operatorname{rk} \pi_{2}>\cdots>\operatorname{rk} \pi_{k}$. We will prove later that minimal factorizations and explicit constructions also give the same condition on the ranks of projections. But the condition we have on the $\pi_{i}^{\prime} s$ is $\operatorname{Im} \pi_{i+1} \cap \operatorname{Im} \pi_{i}^{\perp}=0$ for all $1 \leq i \leq k-1$.

We use the same notation as in Section 5, and assume that the pole $z=i$. Let $a_{0}, a_{1}, \ldots$ be a sequence of $\mathbb{C}^{n}$-valued meromorphic functions on $S^{2}$,

$$
\begin{gathered}
w_{\epsilon}=x+(i+\epsilon) u+(i+\epsilon)^{-1} v, \quad w=x+i y, \\
b_{j, \epsilon}=a_{0}\left(w_{\epsilon}\right)+\epsilon a_{1}\left(w_{\epsilon}\right)+\cdots+\epsilon^{j} a_{j}\left(w_{\epsilon}\right), \\
=c_{0}+c_{1} \epsilon+\cdots+c_{j} \epsilon^{j}+O\left(\epsilon^{j+1}\right),
\end{gathered}
$$

where $c_{j}$ 's are defined by (5.3) with $z=i$. So

$$
\begin{aligned}
c_{0}= & a_{0}(w), \\
c_{1}= & t a_{0}^{\prime}(w)+a_{1}(w), \\
c_{2}= & \frac{t^{2}}{2} a_{0}^{\prime \prime}(w)+i v a_{0}^{\prime}(w)+t a_{1}^{\prime}(w)+a_{2}(w), \\
c_{3}= & \frac{t^{3}}{3 !} a_{0}^{\prime \prime \prime}(w)+i v t a_{0}^{\prime \prime}(w)-v a_{0}^{\prime}(w)+\frac{t^{2}}{2} a_{1}^{\prime \prime}(w)+i v a_{1}^{\prime}(w) \\
& +t a_{2}^{\prime}(w)+a_{3}(w), \\
c_{4}= & \frac{t^{4}}{4 !} a_{0}^{(4)}(w)+\frac{i}{2} v t^{2} a_{0}^{\prime \prime \prime}(w)-\frac{a_{0}^{\prime \prime}(w)}{2}\left(v^{2}+2 v t\right)-i v a_{0}^{\prime}(w) \\
& +\frac{t^{3}}{3 !} a_{1}^{\prime \prime \prime}(w)+i v t a_{1}^{\prime \prime}(w)-v a_{1}^{\prime}(w)+\frac{t^{2}}{2} a_{2}^{\prime \prime}(w)+i v a_{2}^{\prime}(w) \\
& +t a_{3}^{\prime}(w)+a_{4}(w), \\
& \ldots
\end{aligned}
$$

We want to write down the conditions under which the limiting Ward soliton is independent of $t$. Suppose

$$
\phi_{k}=g_{i, \pi_{k}} g_{i, \pi_{k-1}} \cdots g_{i, \pi_{1}}
$$


is a minimal factorization and an extended Ward $k$-soliton obtained by the limiting method of Section 5. Let $V_{j}=\operatorname{Im} \pi_{j}$, and

$$
\begin{aligned}
P_{j i} & =\sum_{j \geq k_{i}>\cdots>k_{1} \geq 1} \pi_{k_{i}}^{\perp} \cdots \pi_{k_{1}}^{\perp}, \\
P_{j, 0} & =\mathrm{I} .
\end{aligned}
$$

Use the computation of Section 5 to conclude that:

1) If $\mathrm{rk} \pi_{2}=1$, then $\phi_{2}$ is independent of $t$ if and only if

$$
\begin{aligned}
& a_{0}, a_{0}^{\prime} \in V_{1}, \\
& v_{2}=a_{0}+2 i \pi_{1}^{\perp}\left(a_{1}\right) .
\end{aligned}
$$

2) If $\operatorname{rk} \pi_{3}=1$, then $\phi_{3}$ is independent of $t$ if and only if

$$
v_{3}=v_{2}+2 i \pi_{2}^{\perp}\left(c_{1}+2 i \pi_{1}^{\perp}\left(c_{2}\right)\right)
$$

is independent of $t$. So the coefficients of $t, t^{2}$ must be zero, which gives

$$
\left\{\begin{array}{l}
\pi_{2}^{\perp} \pi_{1}^{\perp}\left(a_{0}^{\prime \prime}\right)=0 \\
\pi_{2}^{\perp}\left(a_{0}^{\prime}+2 i \pi_{1}^{\perp}\left(\frac{i}{2} a_{0}^{\prime}+a_{1}^{\prime}\right)\right)=0 .
\end{array}\right.
$$

Since $\phi_{2}$ is the minimal factorization, by Proposition 4.8 (2), the first equation implies that $a_{0}^{\prime \prime} \in V_{1}$. We have $a_{0}^{\prime} \in V_{1}$. So the condition for $\phi_{3}$ to be independent of $t$ is

$$
\left\{\begin{array}{l}
a_{0}, a_{0}^{\prime}, a_{0}^{\prime \prime} \in V_{1}, \\
a_{0}+2 i \pi_{1}^{\perp}\left(a_{1}\right), a_{0}^{\prime}+2 i \pi_{1}^{\perp}\left(a_{1}^{\prime}\right) \in V_{2},
\end{array}\right.
$$

and

$$
v_{3}=a_{0}+2 i\left(\pi_{1}^{\perp}+\pi_{2}^{\perp}\right)\left(a_{1}\right)+(2 i)^{2} \pi_{2}^{\perp} \pi_{1}^{\perp}\left(a_{2}\right) .
$$

3) If $\operatorname{rk} \pi_{4}=1$, then $\phi_{4}$ is independent of $t$ if and only if all coefficients of $t, t^{2}, t^{3}$ in

$v_{4}=v_{3}+2 i \pi_{3}^{\perp}\left(c_{1}\right)+(2 i)^{2}\left(\pi_{3}^{\perp} \pi_{1}^{\perp}+\pi_{3}^{\perp} \pi_{2}^{\perp}\right)\left(c_{2}\right)+(2 i)^{3} \pi_{3}^{\perp} \pi_{2}^{\perp} \pi_{1}^{\perp}\left(c_{3}\right)$

are zero. So we get

$$
\begin{cases}a_{0}, a_{0}^{\prime}, a_{0}^{\prime \prime}, a_{0}^{\prime \prime \prime} \in V_{1}, & \\ a_{0}^{(i)}+2 i \pi_{1}^{\perp}\left(a_{1}^{(i)}\right) \in V_{2}, & 0 \leq i \leq 2 \\ a_{0}^{(i)}+2 i\left(\pi_{1}^{\perp}+\pi_{2}^{\perp}\right)\left(a_{1}^{(i)}\right)+(2 i)^{2} \pi_{2}^{\perp} \pi_{1}^{\perp}\left(a_{2}^{(i)}\right) \in V_{3}, & i=0,1\end{cases}
$$

and

$$
v_{4}=a_{0}+(2 i) P_{31}\left(a_{1}\right)+(2 i)^{2} P_{32}\left(a_{2}\right)+(2 i)^{3} P_{33}\left(a_{3}\right) .
$$


4) By induction, if $\operatorname{rk} \pi_{k}=1$, then $\phi_{k}$ is independent of $t$ if and only if

$$
\left\{\begin{array}{l}
a_{0}, \ldots, a_{0}^{(k-1)} \in V_{1}, \\
v_{2}, D^{(1)} v_{2}, \ldots, D^{(k-2)} v_{2} \in V_{2}, \\
\cdots, \\
v_{k-1}, D^{(1)} v_{k-1} \in V_{k-1},
\end{array}\right.
$$

where

$$
D^{(i)} v_{\ell}=\sum_{j=0}^{\ell-1}(2 i)^{j} P_{\ell-1, j}\left(a_{j}^{(i)}\right),
$$

and $a^{(i)}=\frac{d^{i} a}{d w^{i}}$. Thus

$$
v_{k}=\sum_{j=0}^{k-1}(2 i)^{j} P_{k-1, j}\left(a_{j}\right) .
$$

The computation for the case when $\operatorname{rk} \pi_{k} \geq 2$ is similar. In fact, we get

Theorem 9.1. Let $\phi_{k}$ be an extended uniton, $\phi_{k}=g_{i, \pi_{k}} \cdots g_{i, \pi_{1}} a$ minimal factorization, $m_{i}=\operatorname{rk} \pi_{i}$, and

$$
P_{m, j}=\sum_{m \geq i_{1}>\cdots>i_{j} \geq 1} \pi_{i_{1}}^{\perp} \cdots \pi_{i_{j}}^{\perp}
$$

for $1 \leq m \leq k$. Then there exists a partition $\left(r_{1}, \ldots, r_{s}\right)$ of $m_{k}$, (i.e., $r_{j}>0$ and $\left.\sum_{j=1}^{s} r_{j}=m_{k}\right)$ and $\mathbb{C}^{n}$-valued rational maps $a_{i, 0}, \ldots, a_{i, k-1}$ for $1 \leq i \leq s$ such that

1) $a_{i, 0}^{\left(j_{i}\right)} \in \operatorname{Im} \pi_{1}$ for all $0 \leq j_{i} \leq r_{i}+k-2$,

2) $D^{\left(j_{i}\right)} v_{i p} \in \operatorname{Im} \pi_{p}$ for all $0 \leq j_{i} \leq r_{i}+k-p-1$ and $1 \leq p \leq k-1$,

3) $\left\{v_{i k}, D^{(1)} v_{i k}, \ldots, D^{\left(r_{i}-1\right)} v_{i k} \mid 1 \leq i \leq s\right\}$ spans $\operatorname{Im} \pi_{k}$ and $D^{\left(r_{i}\right)} v_{i k} \notin$ $\operatorname{Im} \pi_{k}$,

where

$$
v_{i m}=\sum_{j=0}^{m-1}(2 i)^{j} P_{m-1, j}\left(a_{i, j}\right), \quad D^{(\ell)} v_{i m}=\sum_{j=0}^{m-1}(2 i)^{j} P_{m-1, j} a_{i, j}^{(\ell)} .
$$

Let $\phi_{k}=g_{i, \pi_{k}} \cdots g_{i, \pi_{1}}$ be a minimal factorization and an extended uniton, and $m_{j}=\operatorname{rk}\left(\pi_{j}\right)$. By Proposition $4.8, m_{1} \geq \cdots \geq m_{k}$. We will prove below that $m_{i}$ are strictly decreasing. First note that if $\pi_{i_{0}}$ is constant in the minimal factorization of $\phi_{k}$, then up to a right multiplication in $U(n)$ the corresponding Ward map has an extended solution with $(k-1)$ factors. So we may assume that $\pi_{i}$ is not constant for all $1 \leq i \leq k$. 
Proposition 9.2. Let $\phi_{k}$ be an extended $k$-uniton, $\phi_{k}=g_{i, \pi_{k}} \cdots g_{i, \pi_{1}}$ a minimal factorization, and $m_{i}=\mathrm{rk} \pi_{i}$. If $\pi_{i}$ is not constant for all $1 \leq i \leq k$, then $m_{1}>\cdots>m_{k}$.

Proof. It follows from Proposition 4.8 that $m_{1} \geq m_{2} \geq \cdots \geq m_{k}$. We prove the proposition by induction on $k$. For $k=2$, if the proposition is not true, then $m_{1}=m_{2}$. By Theorem 9.1, there exists a partition $\left(r_{1}, \ldots, r_{s}\right)$ of $m_{2}$ and $\mathbb{C}^{n}$-valued rational maps $a_{i, 0}, a_{i, 1}$ for $1 \leq i \leq s$ so that

(i) $a_{i, 0}^{\left(j_{i}\right)} \in \operatorname{Im} \pi_{1}$ for $0 \leq j_{i} \leq r_{i}$,

(ii) $\left\{D^{\left(j_{i}\right)} v_{i, 2} \mid 1 \leq i \leq s, 0 \leq j_{i} \leq r_{i}-1\right\}$ spans $\operatorname{Im} \pi_{2}$ a.e., where

$$
v_{i 2}=a_{i, 0}+2 i \pi_{1}^{\perp}\left(a_{i, 1}\right), \quad D^{(i)} v_{\ell, 2}=a_{\ell, 0}^{(i)}+2 i \pi_{1}^{\perp}\left(a_{\ell, 1}^{(i)}\right),
$$

(iii) $D^{\left(r_{i}\right)} v_{i, 2} \notin \operatorname{Im} \pi_{2}$.

The definition of minimal factorization implies $\operatorname{Im} \pi_{1}^{\perp} \cap \operatorname{Im} \pi_{2}=0$. So $\pi_{1} \mid V_{2}$ is injective. But $\pi_{1}\left(D^{(j)} v_{i, 2}\right)=a_{i, 0}^{(j)}$ implies that the rank of

$$
Y_{1}=\left\{a_{i, 0}^{\left(j_{i}\right)} \mid 1 \leq i \leq s, 0 \leq j_{i} \leq r_{i}-1\right\}
$$

is $m_{2}$, which is equal to $m_{1}=\operatorname{rk} \pi_{1}$. Thus $Y_{1}$ spans $\operatorname{Im} \pi_{1}$, which implies that $a_{i, 0}^{\left(r_{i}\right)}$ lies in the span of $Y_{1}$. Then a direct computation shows that $\partial_{w} V=h V$ for some meromorphic function $h$, where

$$
V=a_{1,0} \wedge \cdots \wedge a_{1,0}^{\left(r_{1}-1\right)} \wedge \cdots \wedge a_{s, 0} \wedge \cdots \wedge a_{s, 0}^{\left(r_{s}-1\right)} .
$$

Define

$$
f(w)=\exp \left(-\int h(w) d w\right)
$$

locally. Then $\partial_{w}(f V)=0$. But $f V$ is meromorphic. Hence $f V$ is locally constant, which implies that $\pi_{1}$ is a constant Hermitian projection, a contradiction. So $m_{1}>m_{2}$.

Suppose the proposition is true for $k$, and

$$
\phi_{k+1}=g_{i, \pi_{k+1}} \cdots g_{i, \pi_{1}}
$$

satisfies the assumption of the proposition. It follows from Theorem 8.1 that

$$
\phi_{k}=g_{i, \pi_{k}} \cdots g_{i, \pi_{1}}
$$

is also an extended uniton. It is easy to check that $\phi_{k}$ also satisfies the conditions of the proposition. So by the induction hypothesis, $m_{1}>$ $\cdots>m_{k}$. We already have $m_{k} \geq m_{k+1}$ by Proposition 4.8. We will show that $m_{k}=m_{k+1}$ gives a contradiction next. By Theorem 9.1, there exist a partition $\left(r_{1}, \ldots, r_{s}\right)$ of $m_{k+1}$ and $a_{i, j}$ 's so that $\left\{D^{\left(j_{i}\right)} v_{i, k+1} \mid 1 \leq\right.$ $\left.i \leq s, 0 \leq j_{i} \leq r_{i}-1\right\}$ form a basis of $\operatorname{Im} \pi_{k+1}$. Use

$$
P_{k, j}=P_{k-1, j}+\pi_{k}^{\perp} P_{k-1, j-1}
$$


to see that $D^{(\ell)} v_{i, k+1}=D^{(\ell)} v_{i, k}+\xi_{i, k, \ell}$ for some $\xi_{i, k, \ell}$ in $\operatorname{Im} \pi_{k}^{\perp}$. Since $\operatorname{Im} \pi_{k+1} \cap \operatorname{Im} \pi_{k}^{\perp}=0$ and $\pi_{k} \mid \operatorname{Im} \pi_{k+1}$ is injective,

$$
Y_{k}=\left\{D^{\left(\ell_{i}\right)} v_{i, k} \mid 1 \leq i \leq s, 0 \leq \ell_{i} \leq r_{i}-1\right\}
$$

has rank $m_{k+1}$, which is equal to $m_{k}$. But $D^{\left(r_{i}\right)} v_{i, k} \in \operatorname{Im} \pi_{k}$. So a similiar argument as in the case $k=2$ implies that $\pi_{k}$ is a constant, a contradiction.

q.e.d.

We give some examples to demonstrate how to write down unitons from rational maps.

Example 9.3. An extended 4-uniton $\phi_{4}=g_{i, \pi_{4}} \cdots g_{i, \pi_{1}}$ in $U(5)$ with rank data $(4,3,2,1)$ is given by $\mathbb{C}^{5}$-valued rational maps $a_{0}, a_{1}, a_{2}, a_{3}$ such that

1) $\operatorname{Im} \pi_{1}$ is spanned by $a_{0}, \ldots, a_{0}^{(3)}$,

2) $\operatorname{Im} \pi_{2}$ is spanned by $a_{0}^{(j)}+2 i \pi_{1}^{\perp}\left(a_{1}^{(j)}\right)$ with $0 \leq j \leq 2$,

3) $\operatorname{Im} \pi_{3}$ is spanned by $a_{0}^{(j)}+2 i P_{21}\left(a_{1}^{(j)}\right)+(2 i)^{2} P_{22}\left(a_{2}^{(j)}\right)$ with $j=0,1$,

4) $\operatorname{Im} \pi_{4}$ is spanned by $v_{4}$ defined by $(9.3)$.

Note that $a_{0}$ should be chosen so that $a_{0}, a_{0}^{\prime}, \ldots, a_{0}^{(4)}$ are linearly independent a.e., otherwise $\pi_{1}$ is constant.

Example 9.4. A 3 -uniton $\phi_{3}=g_{i, \pi_{3}} g_{i, \pi_{2}} g_{i, \pi_{1}}$ in $U(5)$ with rank data $(4,2,1)$ is given by $\mathbb{C}^{5}$-valued rational maps $a_{0}, b_{0}, a_{1}, a_{2}$

1) $\operatorname{Im} \pi_{1}$ is spanned by $a_{0}, a_{0}^{\prime}, a_{0}^{\prime \prime}, b_{0}$,

2) $\operatorname{Im} \pi_{2}$ is spanned by $a_{0}+2 i \pi_{1}^{\perp}\left(a_{1}\right), a_{0}^{\prime}+2 i \pi_{1}^{\perp}\left(a_{1}^{\prime}\right)$,

3) $\operatorname{Im} \pi_{3}$ is spanned by $\sum_{j=0}^{2}(2 i)^{j} P_{2 j}\left(a_{j}\right)$.

Example 9.5. 3-unitons in $U(6)$ with rank data $(5,3,1)$.

Case 1. Choose $\mathbb{C}^{6}$-valued rational maps $a_{0}, a_{1}, a_{2}, b_{0}, b_{1}$ on $\mathbb{C}$ such that

1) $a_{0}, a_{0}^{\prime}, a_{0}^{\prime \prime}, b_{0}, b_{0}^{\prime}$ are linearly independent a.e., and their span intersects the space $\mathbb{C}^{n}$ of constant maps from $\mathbb{C}$ to $\mathbb{C}^{n}$ only at 0 ,

2) $v_{2}=a_{0}+2 i \pi_{1}^{\perp}\left(a_{1}\right), D^{(1)} v_{2}=a_{0}^{\prime}+2 i \pi_{1}^{\perp}\left(a_{1}^{\prime}\right), \hat{v}_{2}=b_{0}+2 i \pi_{1}^{\perp}\left(b_{1}\right)$ are linearly independent a.e.,

3) $v_{3}=a_{0}+2 i P_{21}\left(a_{1}\right)+(2 i)^{2} P_{22}\left(a_{2}\right)$ is not zero a.e..

Let $\pi_{1}, \pi_{2}$, and $\pi_{3}$ be the projections of $\mathbb{C}^{6}$ onto the span of $a_{0}, a_{0}^{\prime}, a_{0}^{\prime \prime}$, $b_{0}, b_{0}^{\prime}$, the span of $v_{2}, D^{(1)} v_{2}, \hat{v}_{2}$, and $\mathbb{C} v_{3}$ respectively. Then $\phi_{3}=$ $g_{i, \pi_{3}} g_{i, \pi_{2}} g_{i, \pi_{1}}$ is an extended solution of a 3 -uniton.

Case 2. Choose $\mathbb{C}^{6}$-valued rational maps $a_{0}, b_{0}, a_{1}, a_{2}$ on $\mathbb{C}$ such that

1) $\operatorname{Im} \pi_{1}$ is spanned by $a_{0}, a_{0}^{\prime}, a_{0}^{\prime \prime}, a_{0}^{\prime \prime \prime}, b_{0}$,

2) $\operatorname{Im} \pi_{2}$ is spanned by $a_{0}+2 i \pi_{1}^{\perp}\left(a_{1}\right), a_{0}^{\prime}+2 i \pi_{1}^{\perp}\left(a_{1}^{\prime}\right), a_{0}^{\prime \prime}+2 i \pi_{1}^{\perp}\left(a_{1}^{\prime \prime}\right)$,

3) $\operatorname{Im} \pi_{3}$ is spanned by $f_{0} v_{3}+f_{1} D^{(1)} v_{3}$, where $v_{3}=\sum_{j=0}^{2}(2 i)^{j} P_{2 j}\left(a_{j}\right)$, $D^{(1)} v_{3}=\sum_{j=0}^{2}(2 i)^{j} P_{2 j}\left(a_{j}^{\prime}\right)$, and $f_{0}, f_{1}: \mathbb{C} \rightarrow \mathbb{C}$ are rational functions.

These two cases give all 3 -unitons in $U(6)$ with rank data $(5,3,1)$. 


\section{Wave profiles of multi-solitons}

We give the wave profiles of some $k$-solitons by showing the graphs of their energy densities $E\left(x, y, t_{i}\right)$ suitably scaled for a sequence of increasing times $t_{i}$. Each figure shows the graph of $z=s E\left(x, y, t_{i}\right)$ over the domain $\{(x, y)|| x|\leq r| y \mid, \leq r\}$. The values of $r, s$ are given under each figure. Since most poles are chosen so that the corresponding solitons are moving from right to left, we show the profile from right to left as time increases.

A soliton with pole data $\left(z_{1}, \ldots, z_{r}, n_{1}, \ldots, n_{r}\right)$ can be regarded as the interaction of $r$ solutions that separate when $|t|$ is large. This is very similar to the behavior of soliton equation in one space and one time variables. But it is difficult to predict the dynamics of solitons with pole data $(z, k)$.

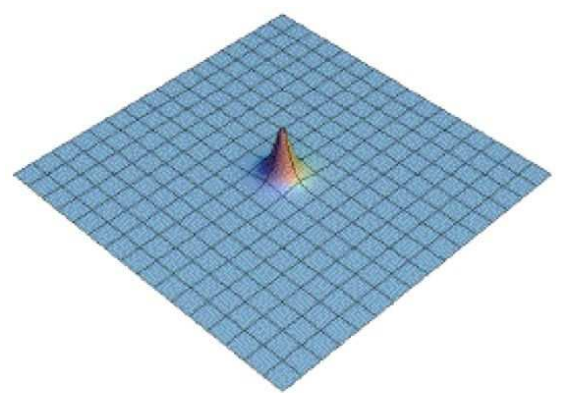

(a)

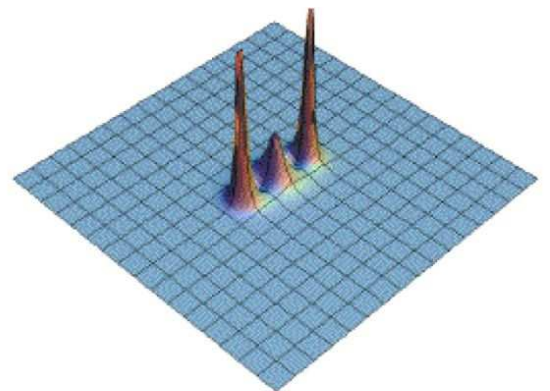

(b)

Figure 1. 1-solitons, $r=6, s=0.05$.

Figure 1(a) and 1(b) are the profiles of 1-solitons at $t=0$, whose extended solutions are $g_{i, \pi_{1}}$ and $g_{1+i, \pi_{2}}$ respectively. Here $\pi_{1}$ and $\pi_{2}$ are the projections of $\mathbb{C}^{2}$ onto $\mathbb{C}(1,2 w)^{T}$ and onto $\mathbb{C}\left(1,2 w+w^{3}\right)^{T}$ respectively. Note that $w$ depends on the pole location of the simple element $g_{z, \pi}$, i.e., $w=x+z u+z^{-1} v=x+\frac{z-z^{-1}}{2} y+\frac{z+z^{-1}}{2} t$. The travelling velocity of the corresponding 1 -soliton depends on the pole $z=r e^{i \theta}$ given by (2.5). The 1-soliton given in $1(\mathrm{a})$ is stationary. The 1-soliton given in $1(\mathrm{~b})$ is the travelling wave in the direction $\left(-1,-\frac{1}{2}\right)^{T}$ as shown in Figure 2 below.

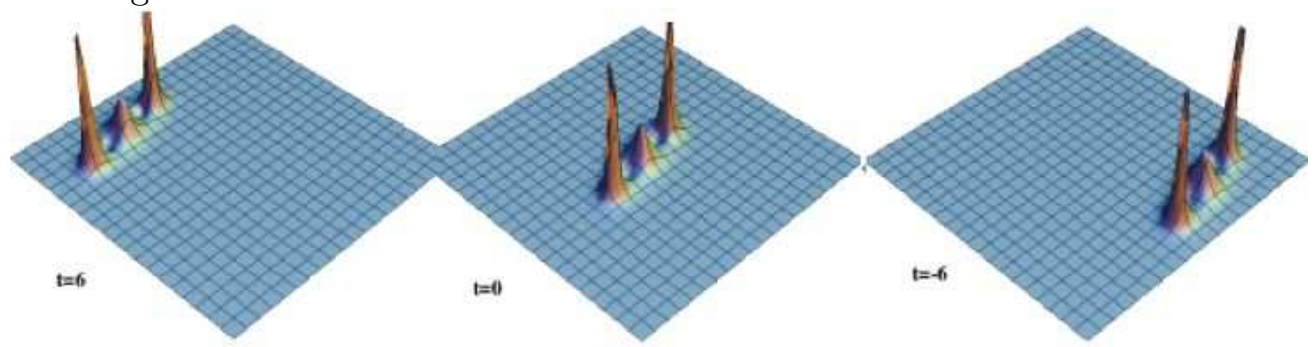

Figure 2. 1-soliton with a pole at $1+i, r=6, s=0.05$. 
Figure 3 below shows the wave profiles of the 2-soliton whose extended solution is $g_{1+i, \pi_{2}} * g_{i, \pi_{1}}$, where $\pi_{1}$ and $\pi_{2}$ are as in Figure 1 . This represents the interaction of the two 1 -solitons given by $g_{i, \pi_{1}}$ (the small single lump in the middle) and $g_{1+i, \pi_{2}}$ (the travelling three lumps in the right). When $t<-4$, the two 1 -solitons are separate, they interact when $|t| \leq 4$, and then separate again when $t>4$, but a phase shift occurs.

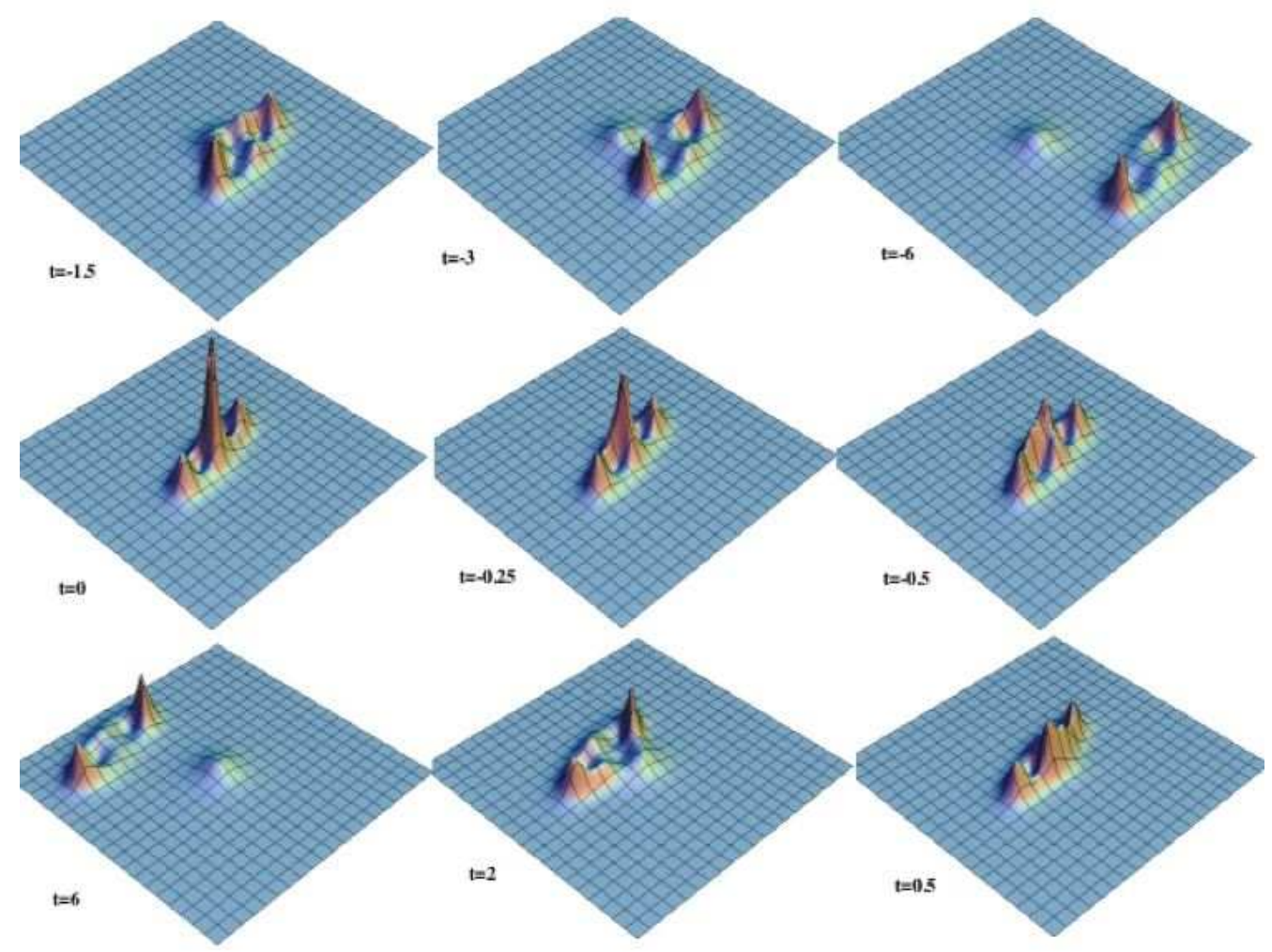

Figure 3. 2-solitons with poles at $i, i+1, r=6, s=0.05$.

Figures 4 and 5 demonstrate the limiting method by showing the wave profiles of 2-solitons whose extended solutions are $g_{\epsilon+i, \pi_{\epsilon}} * g_{i, \pi_{1}}$, where $\pi_{1}$ and $\pi_{\epsilon}$ are projections of $\mathbb{C}^{2}$ onto $v_{1}=(1,2 w)^{T}$ and $v_{2}=\left(1,2 w+\epsilon w^{3}\right)^{T}$ respectively. Figure 3 is the 2 -soliton with $\epsilon=1$. When $0<\epsilon<0.5$, the profile of the 1-soliton given by $g_{\epsilon+i, \pi_{\epsilon}}$ has three lumps on a line with equal distance $d(\epsilon)$ apart. As $\epsilon$ becomes smaller, the distance $d(\epsilon)$ becomes bigger. For example, $d(0.1) \approx 4.5$ and $d(0.001) \approx 60$. However, when $\epsilon$ is small, the 2-soliton given by $g_{\epsilon+i, \pi_{\epsilon}} * g_{i, \pi_{1}}$ does not keep the profile of 1-solitons given by $g_{i, \pi_{1}}$ and $g_{\epsilon+i, \pi_{\epsilon}}$. 
Figure $4(\epsilon=0.1)$ is the 2 -soliton with extended solution $g_{i+0.1, \pi_{0.1}} *$ $g_{i, \pi_{1}}$, where $\pi_{1}$ is the projection onto $\mathbb{C}(1,2 w)^{T}$ and $\pi_{2}$ the projection onto $\mathbb{C}\left(1,2 w+0.1 w^{3}\right)^{T}$. When $t<-20$, the three lumps behave like travelling waves. When $t \in[-20,20]$, the three lumps move to the middle to form a single lump, then separate and move out again. When $t>20$, the three lumps travel along three straight lines and behave like travelling waves. The smaller lump moves faster, and the two taller lumps move at the same speed when $|t|$ is large.

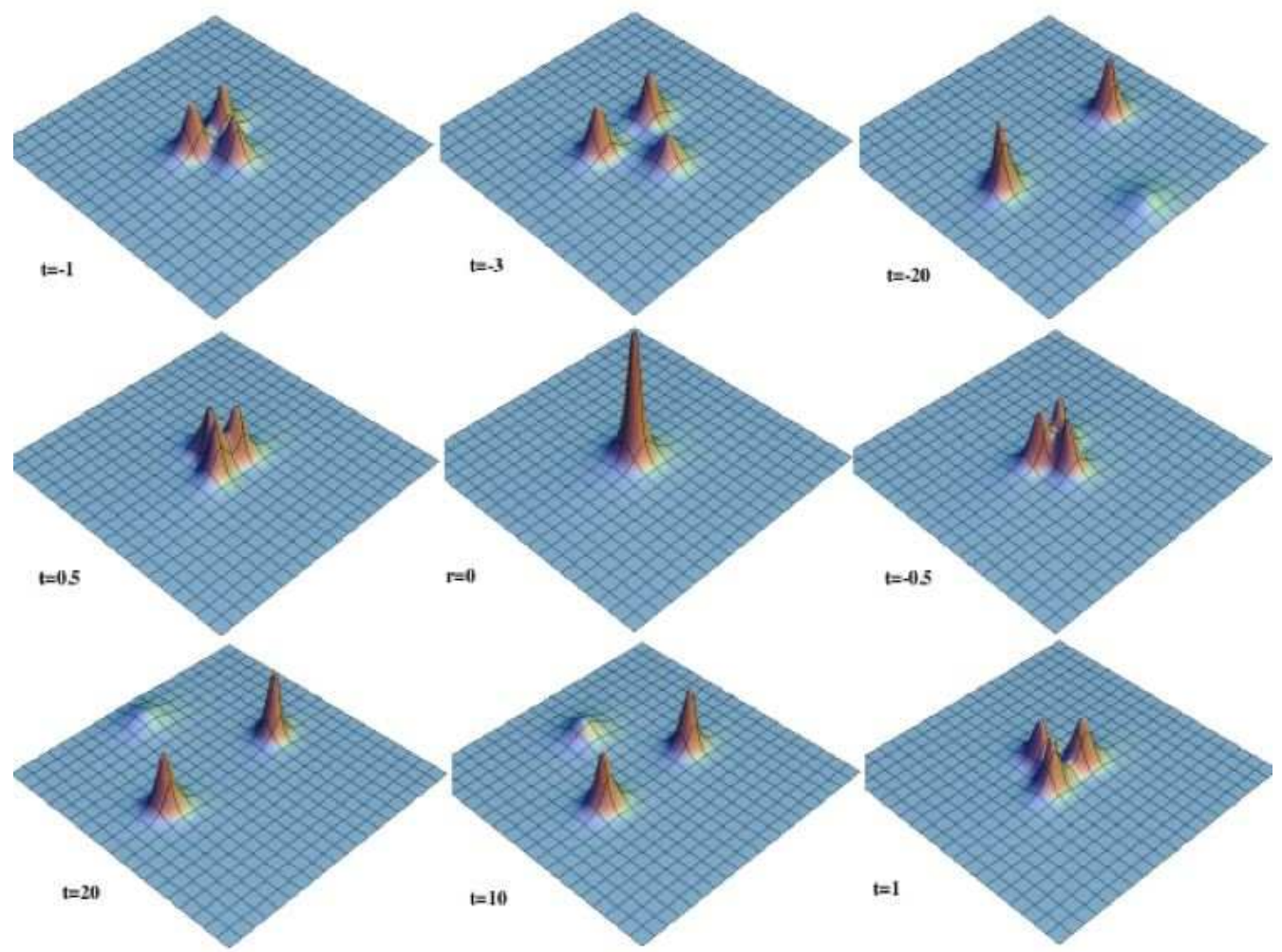

Figure 4. 2-solitons with poles at $i, i+0.1, r=6, s=0.05$. 
Figure 5 is the 2 -soliton with extended solution $g_{0.0001+i, \pi_{0.0001}} * g_{i, \pi_{1}}$, where $\pi_{1}$ and $\pi_{2}$ are the projections onto $\mathbb{C}(1,2 w)^{T}$ and $\mathbb{C}(1,2 w+$ $\left.0.0001 w^{3}\right)^{T}$ respectively. The three lumps move to the middle to form a single lump, then separate to three lumps and move out, but make a $\frac{\pi}{3}$ turn. This is essentially the limiting 2 -soliton with a double pole at $i$ constructed using $a_{0}=2 w, a_{1}=w^{3}$ in Section 5 .

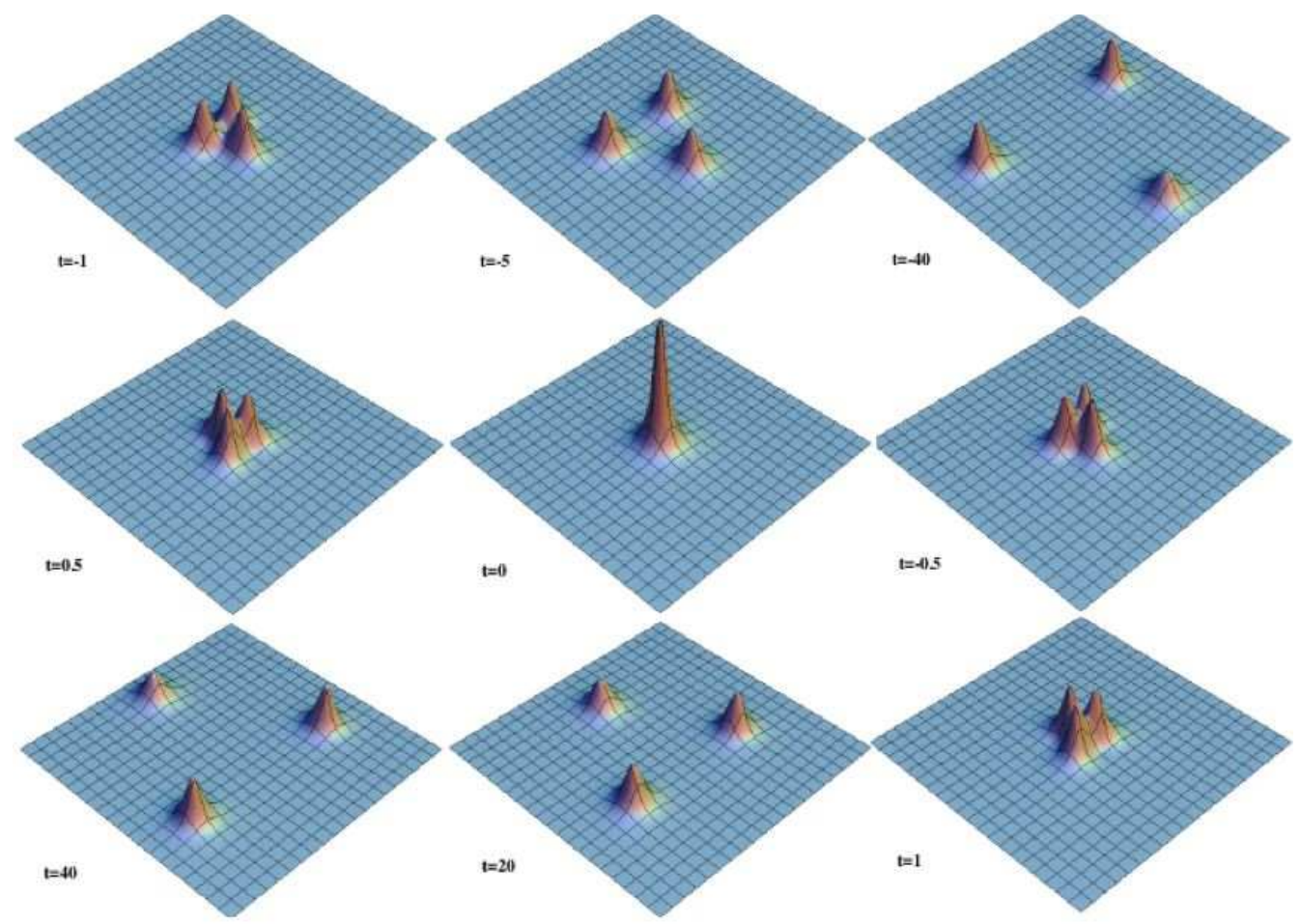

Figure 5. 2-soliton with poles at $i, i+0.001, r=6, s=0.05$. 
Figure 6 is the 2-soliton constructed by the limiting method of Section 5 with the double pole at $1+i$ and $a_{0}=2 w, a_{1}=w^{3}$. It is like the travelling wave of the 2 -soliton with a double pole at $i$ with the same $a_{0}, a_{1}$ as shown in Figure 5 .

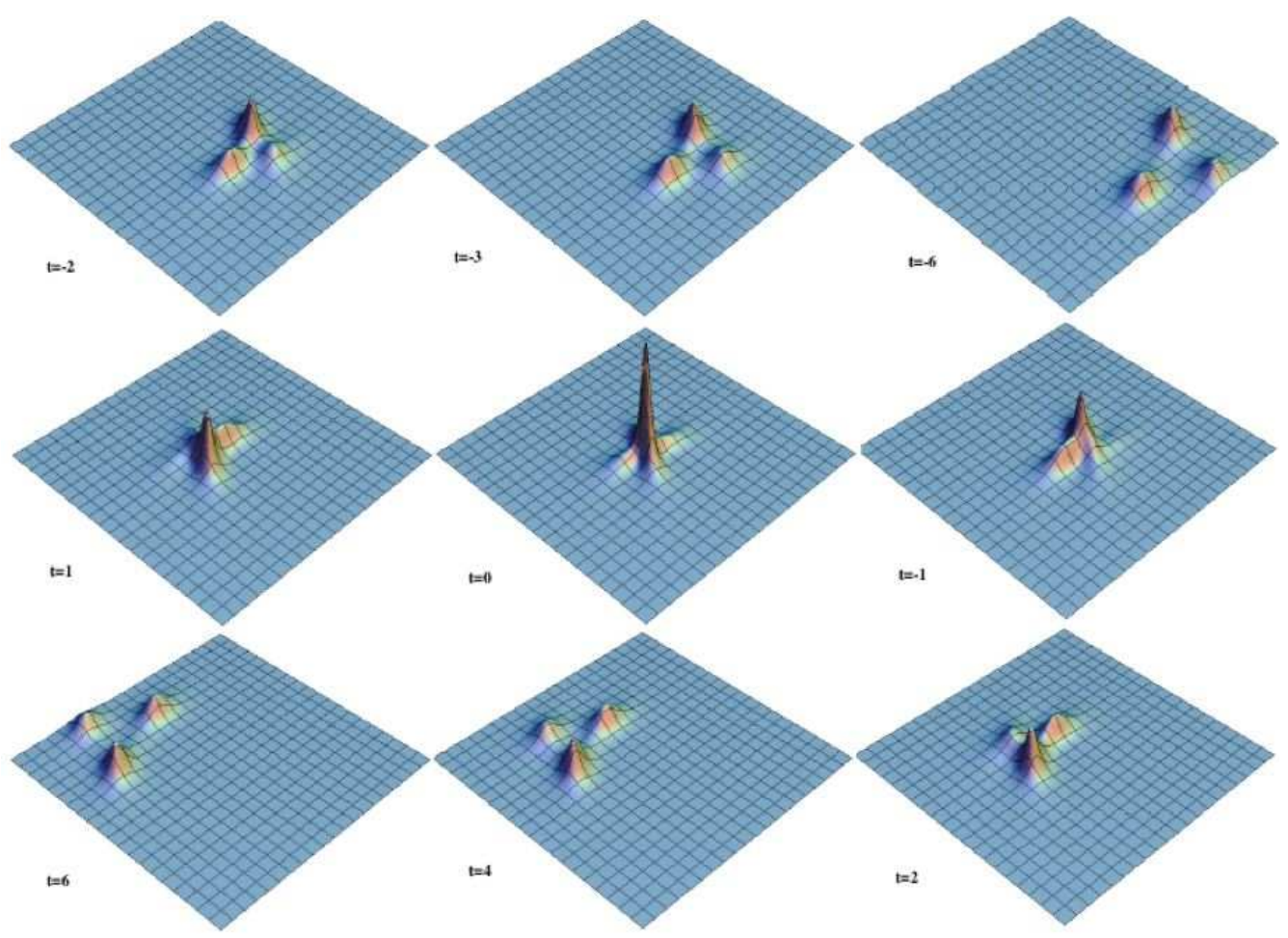

Figure 6. 2 -soliton with a double pole at $1+i, r=6, s=0.1$. 
Figure 7 is the 3 -soliton with a double pole at $i$ and a simple pole at $1+i$ given by $g_{1+i, \pi_{3}} * \psi$, where $\psi$ is the limiting 2 -soliton with a double pole at $i$ with $a_{0}=w, a_{1}=w^{3}$ constructed in Section 5 , and $\pi_{3}$ is the projection onto the linear span of $\left(1, w^{2}\right)^{T}$. At $t=-8$, the 2 -soliton given by $\psi$ is the three tall lumps in the middle, and the 1 -soliton is the travelling two lumps on the right. The interaction of the 2-soliton and 1-soliton happens between $t=-5$ and $t=5$. When $|t| \geq 6$, the two solitons separate as if there had been no interactions, except that a phase shift occurs.

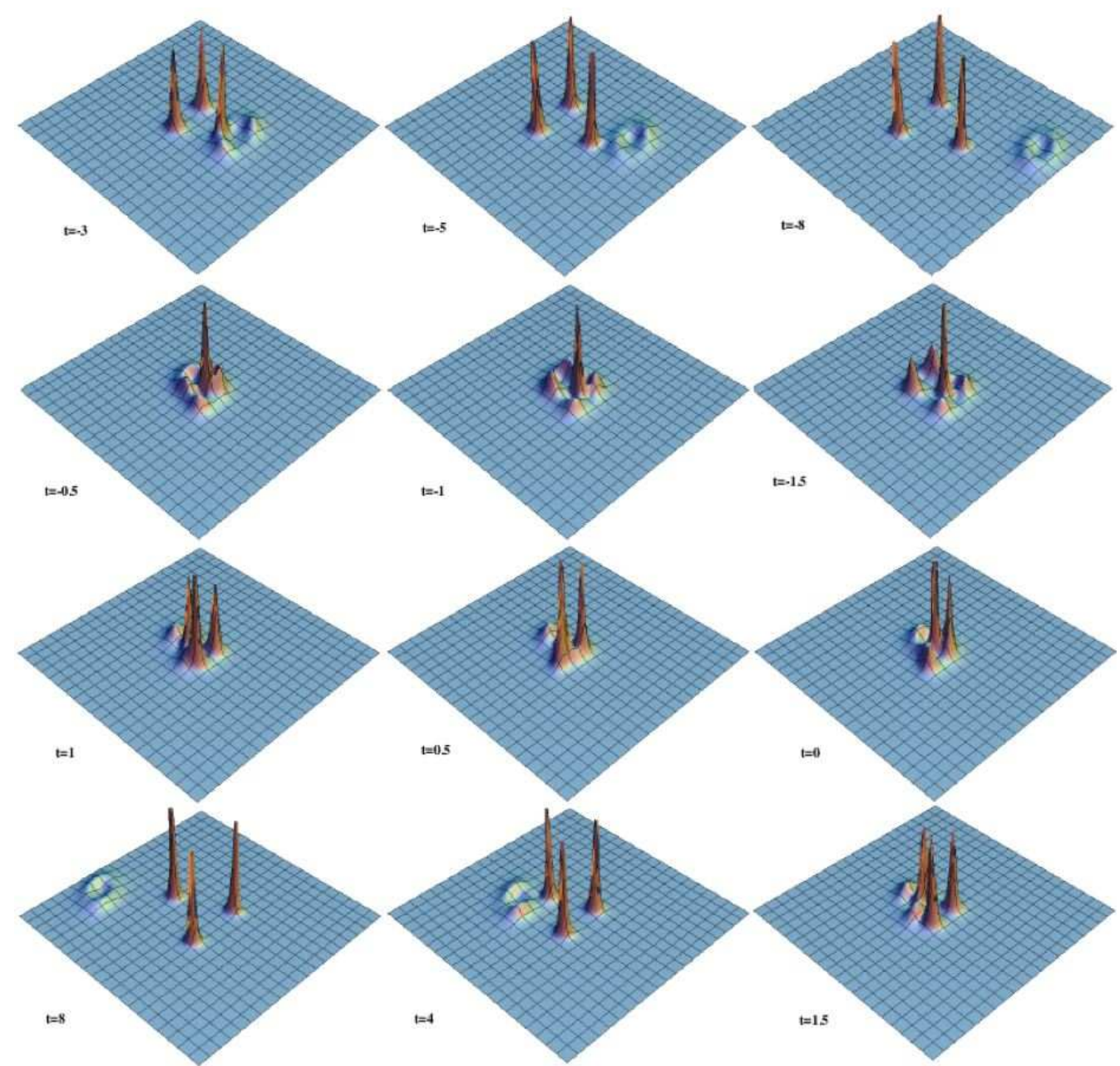

Figure 7. 3 -soliton with a double pole at $i$ and a simple pole at $1+i$, $r=7, s=0.03$. 
Figure 8 is the 3 -soliton with a triple pole at $i$ constructed using $a_{0}(w)=w, a_{1}(w)=w^{3}, a_{2}(w)=w^{5}$ as in Section 5. At $t=-8$, there are 6 lumps forming a triangular shape. Most interactions occur from $t=-3$ to $t=3$, and the triangular shape rotates $\frac{\pi}{3}$ after interaction. When $|t|>8$, the three lumps at the vertices of the triangle spread out but the three lumps at the midpoints of the edges of the triangle do not spread out.

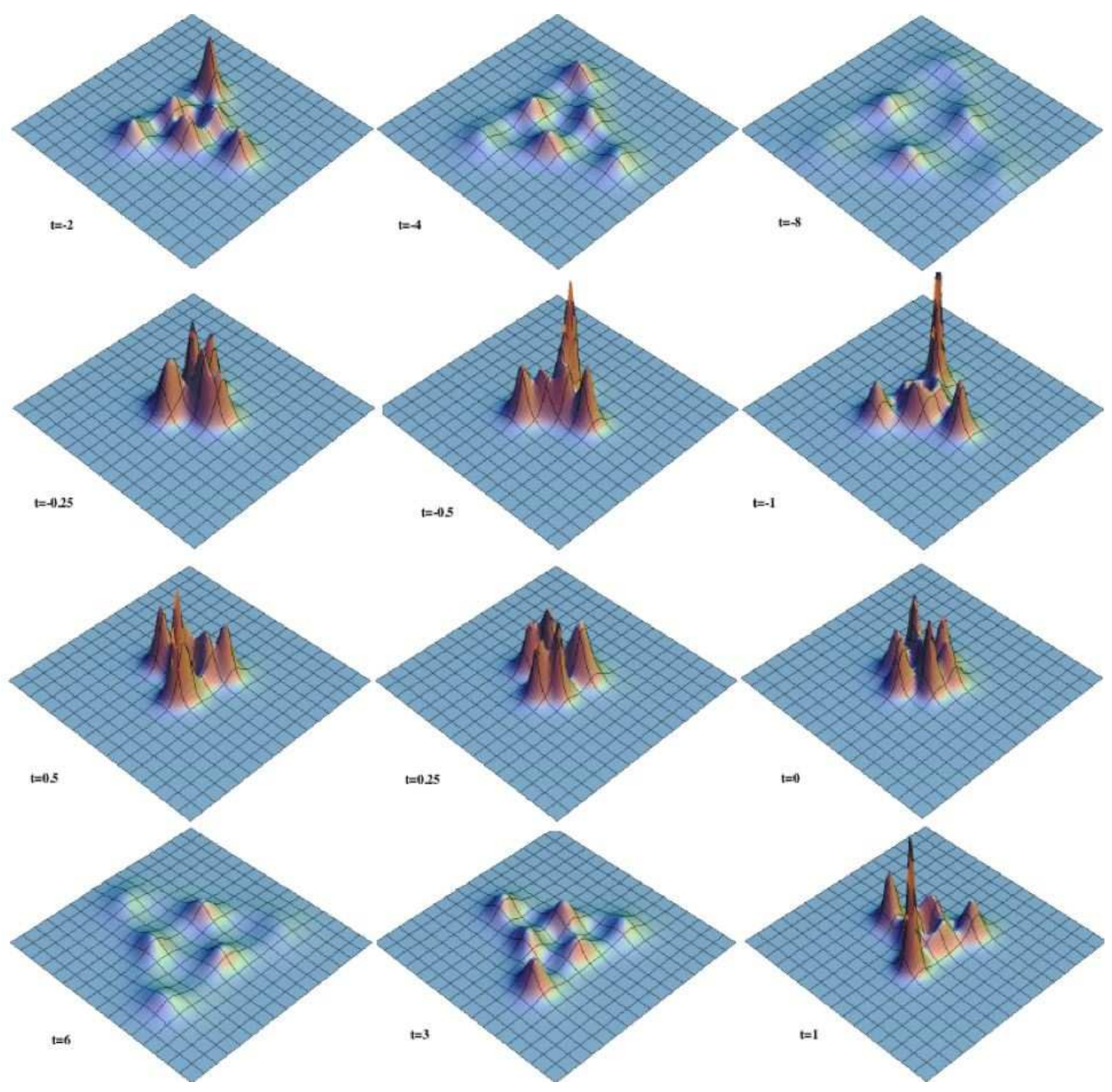

Figure 8. 3-soliton with a triple pole at $i, r=4, s=0.02$. 
Figure 9 is the 4 -soliton with extended solution $g_{0.5+0.75 i, \pi_{4}} *\left(g_{1+i, \pi_{3}} *\right.$ $\left.\psi_{2}\right)$, where $\psi_{2}$ is the 2 -soliton with a double pole at $i, a_{0}=w, a_{1}=w^{3}$, constructed by the limiting method in Section 5 , and $\pi_{3}$ and $\pi_{4}$ are the projections onto $\mathbb{C}\left(1,4 w^{2}+1\right)^{T}$ and $\mathbb{C}\left(1,0.5 w^{4}+1\right)^{T}$ respectively. After interaction, the 2 -soliton given by $\psi_{2}$ (the three lumps in the middle) and the two 1-solitons given by $\pi_{3}$ (two lumps) and $\pi_{4}$ (4 lumps) behave as if there had been no interaction except a possible phase shift.

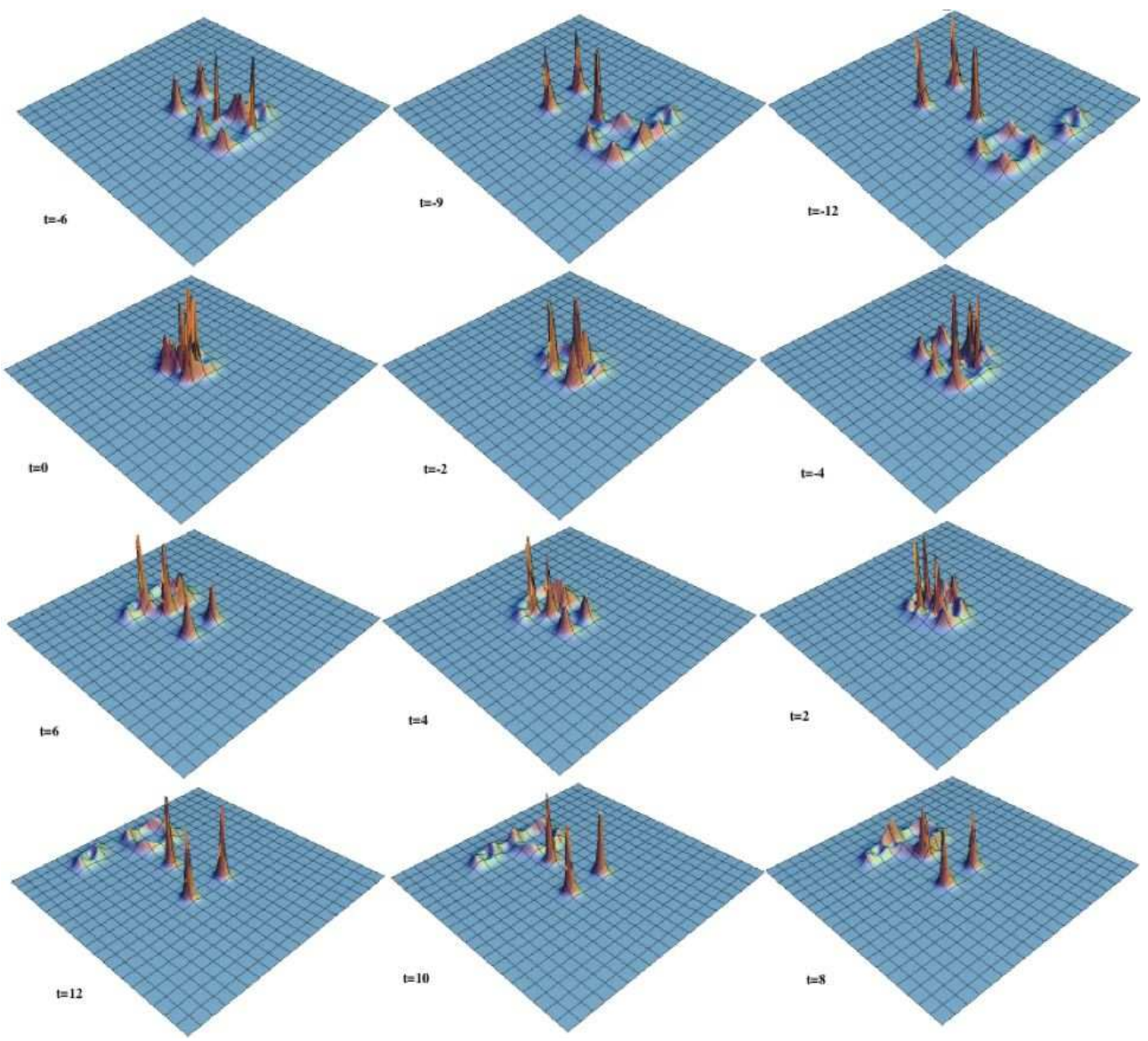

Figure 9. 4-soliton with poles $i, i, 1+i, 0.5+0.75 i, r=10, s=0.04$. 
Figure 10 is the 4 -soliton whose extended solution is $\psi_{2} * \psi_{1}$, where $\psi_{1}$ is the limiting extended 2-soliton with a double pole at $i$ with $a_{0}=w$, $a_{1}=w^{3}$, and $\psi_{2}$ is the limiting 2 -soliton with a double pole at $1+i$ with $a_{0}=w^{4}$ and $a_{1}=w^{2}+w+1$ constructed in Section 5. At $t=-9$, the 2 -soliton given by $\psi_{1}$ is the middle three lumps, and the 2 -soliton given by $\psi_{2}$ is the ring with three lumps in the middle on the right. These 2-solitons keep their shape after interaction, but with a phase shift.

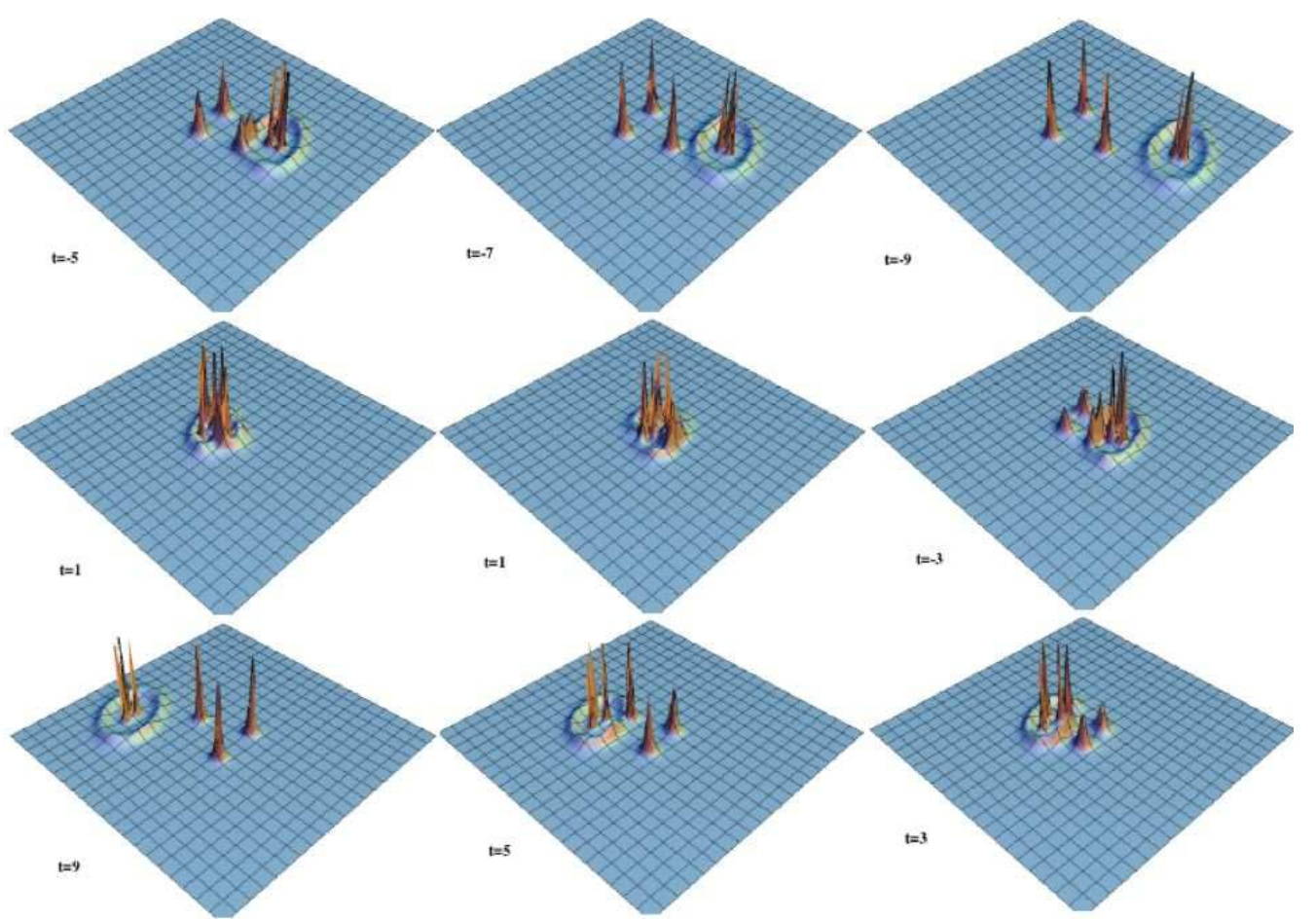

Figure 10. 4 -soliton with double poles at $i$ and $1+i, r=10, s=0.03$. 
Figure 11 is the 4 -soliton whose extended solution is $g_{1+i, \pi_{4}} * \psi_{3}$, where $\psi_{3}$ is the extended solution of the 3 -soliton with a triple pole at $i$ shown in Figure 8 and $\pi_{4}$ is the projection onto $\left(1, w^{2}\right)^{t}$. Note the 3 -soliton and 1-soliton separate after interaction, and the 1-soliton has a phase shift.

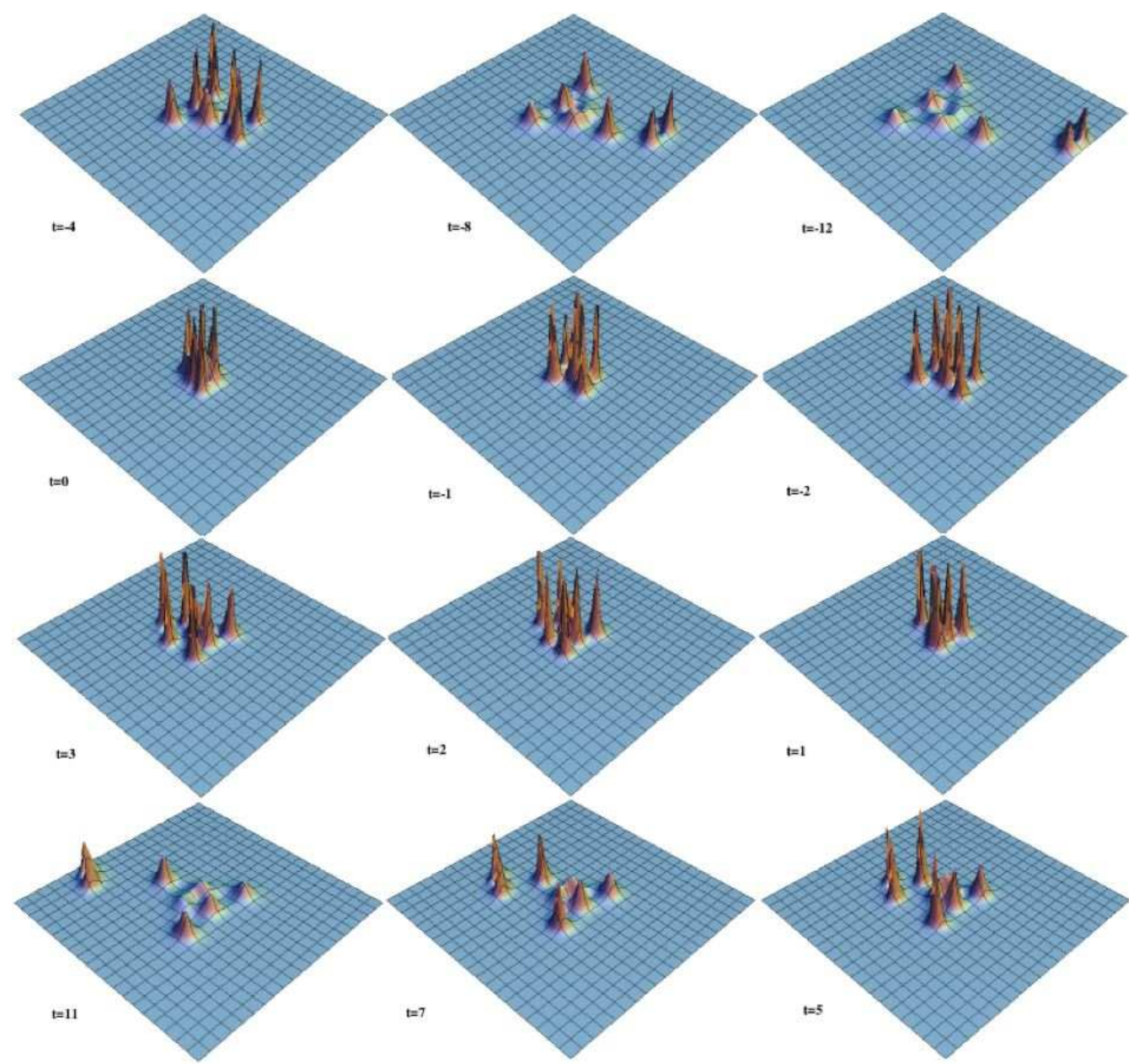

Figure 11. 4 -soliton with poles $i, i, i, 1+i, r=9, s=0.05$. 
Figure 12 is the 4 -soliton with an order 4 pole at $i, a_{0}=w, a_{1}=w^{5}$, $a_{3}=w^{4}$, and $a_{4}=w^{2}$, constructed by the limiting method of Section 5. There are five smaller lumps in the outer ring, five tall lumps in the middle ring, and a well with three lumps in the center ring. After the interaction, the lumps in the two outer rings rotate $\frac{\pi}{5}$ and the three lumps in the center ring rotate $\frac{\pi}{3}$.

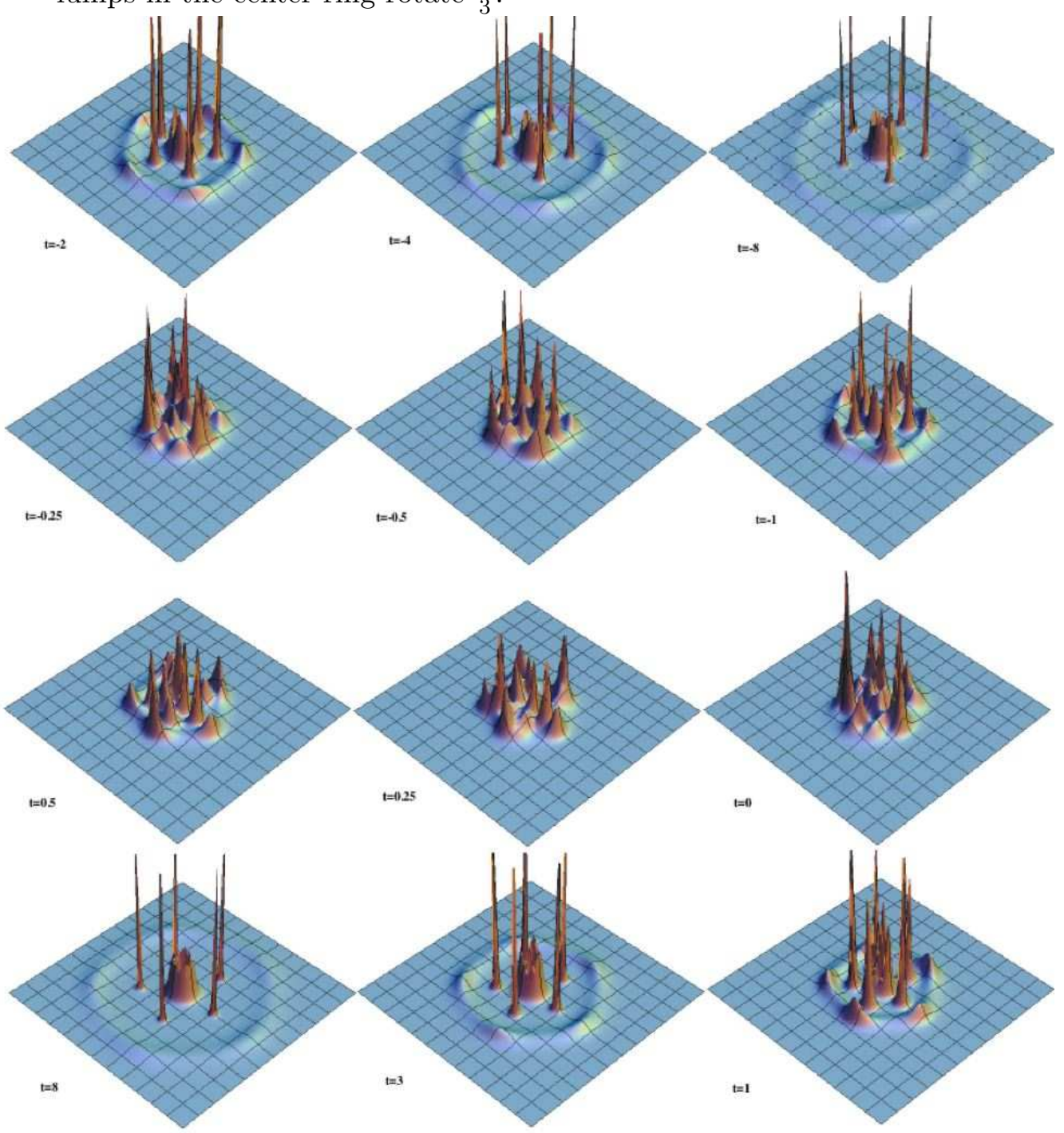

Figure 12. 4-solitons with a quadruple pole at $i, r=4, s=0.005$. 
The quick time movies of the wave motions given in this section can be seen on

http://math.uci.edu/ cterng/WardSolitonMovies.html.

\section{References}

[1] C.K. Anand, Ward's solitons, Geom. Topol. 1 (1997) 9-20, MR 1469065, Zbl 0886.35130.

[2] _ Ward's solitons II, Exact solutions, Canad. J. Math. 50 (1998) 1119 1137, MR 1657763, Zbl 0935.35128.

[3] M.J. Bergvelt \& M. Guest, Actions of loop groups on harmonic maps, Trans. Amer. Math. Soc. 326 (1991) 861-886, MR 1062870, Zbl 0745.58015.

[4] F.E. Burstall \& M.A. Guest, Harmonic two-spheres in compact symmetric spaces, revisited, Math. Ann. 309 (1997) 541-572, MR 1483823, Zbl 0897.58012.

[5] A.S. Fokas \& T.A. Ioannidou, The inverse spectral theory for the Ward equation and for the $2+1$ chiral model, Commun. Appl. Anal. 5(2) (2001) 235-246, MR 1844193.

[6] T. Ioannidou, Soliton solutions and nontrivial scattering in an integrable chiral model in $(2+1)$ dimensions, J. Math. Phys. 37 (1996) 3422-3441, MR 1401233, Zbl 0863.58034 .

[7] T. Ioannidou \& R.S. Ward, Conserved quantities for integrable chiral model in $2+1$ dimensions, Phys. Lett. A 208 (1995) 209-213, MR 1363152, Zbl 1020.37531.

[8] T. Ioannidou \& W. Zakrzewski, Solutions of the modified chiral model in $(2+1)$ dimensions, J. Math. Phys. 39(5) (1998) 2693-2701, MR 1621453, Zbl 1001.58013.

[9] C.L. Terng \& K. Uhlenbeck, Bäcklund transformations and loop group actions, Comm. Pure Appl. Math. 53 (2000) 1-75, MR 1715533, Zbl 1031.37064.

[10] K. Uhlenbeck, Harmonic maps into Lie groups (classical solutions of the chiral model), J. Differential Geom. 30 (1989) 1-50, MR 1001271, Zbl 0677.58020.

[11] J. Villarroel, The inverse problem for Ward's system, Stud. Appl. Math. 83 (1990) 211-222, MR 1071360, Zbl 0737.35055.

[12] R.S. Ward, Soliton solutions in an integrable chiral model in $2+1$ dimensions, J. Math. Phys. 29 (1988) 386-389, MR 0927022, Zbl 0644.58038.

[13] _ Classical solutions of the chiral model, unitons, and holomorphic vector bundles, Commun. Math. Phys. 128 (1990) 319-332, MR 1043523, Zbl 0707.32007.

[14] Nontrivial scattering of localized solutions in a $(2+1)$-dimensional integrable systems, Phys. Lett. A 208 (1995) 203-208, MR 1363151, Zbl 1020.37537.

[15] J.C. Wood, Explicit construction and parametrization of harmonic maps into the unitary group, Proc. London Math. Soc. 58 (1989) 608-624, MR 0988105, Zbl 0679.58015 . 
[16] Z.X. Zhou, Construction of explicit solutions of modified principal chiral field in $1+2$ dimensions via Darboux transformations, in 'Differential Geometry' (C.H. Gu et al, eds.), 325-332, World Scientific, 1993, MR 1341626, Zbl 0787.35093.

LMAM

School of Mathematical Sciences

PEKING UNIVERSITY

Beijing 100871, People's Republic of China

E-mail address: daibo@math.pku.edu.cn

NORTHEASTERN UNIVERSITY

Boston, MA 02115

and

University of CALifornia AT IRVine

IRvine, CA 92697

E-mail address: cterng@math.uci.edu 